\title{
TheOry of $\mathfrak{g}-\mathfrak{T}_{\mathfrak{g}}$-SePARATION Axioms
}

Khodabocus M. I. AND SOOKIA N. U. H.

\begin{abstract}
Several specific types of ordinary and generalized separation axioms of a generalized topological space have been defined and investigated for various purposes from time to time in the literature of topological spaces. Our recent research in the field of a new class of generalized separation axioms of a generalized topological space is reported herein as a starting point for more generalized classes.
\end{abstract}

KEY WORDS AND PHRASES. Generalized topological space, generalized separation axioms, generalized sets, generalized operations

\section{INTRODUCTION}

Whether it concerns the theory of $\mathcal{T}$-spaces or $\mathcal{T}_{\mathfrak{g}}$-spaces, the idea of adding a $\mathrm{T}_{\alpha}$ or a $\mathfrak{g}-\mathrm{T}_{\alpha}$-axiom ${ }^{1}$ (with $\alpha=0,1,2, \ldots$ ) to the axioms for a $\mathcal{T}$-space $\mathfrak{T}=(\Omega, \mathcal{T})$ to obtain a $\mathcal{T}^{(\alpha)}$-space $\mathfrak{T}^{(\alpha)}=\left(\Omega, \mathcal{T}^{(\alpha)}\right)$ or a $\mathfrak{g}-\mathcal{T}^{(\alpha)}$-space $\mathfrak{g}$ - $\mathfrak{T}^{(\alpha)}=\left(\Omega, \mathfrak{g}-\mathcal{T}^{(\alpha)}\right)$ or, the idea of adding a $\mathrm{T}_{\mathfrak{g}, \alpha}$ or a $\mathfrak{g}-\mathrm{T}_{\mathfrak{g}, \alpha}$-axiom (with $\alpha=0,1,2, \ldots$ ) to the axioms for a $\mathcal{T}_{\mathfrak{g}}$-space $\mathfrak{T}_{\mathfrak{g}}=\left(\Omega, \mathcal{T}_{\mathfrak{g}}\right)$ to obtain a $\mathcal{T}_{\mathfrak{g}}^{(\alpha)}$-space $\mathfrak{T}_{\mathfrak{g}}^{(\alpha)}=\left(\Omega, \mathcal{T}_{\mathfrak{g}}^{(\alpha)}\right)$ or a $\mathfrak{g}-\mathcal{T}_{\mathfrak{g}}^{(\alpha)}$ space $\mathfrak{g}-\mathfrak{T}_{\mathfrak{g}}^{(\alpha)}=\left(\Omega, \mathfrak{g}-\mathcal{T}_{\mathfrak{g}}{ }^{(\alpha)}\right)$ has never played little role in Generalized Topology and Abstract Analysis [17, 19, 25]. Because the defining attributes of a $\mathcal{T}$-space in terms of a collection of $\mathcal{T}$-open or $\mathfrak{g}$ - $\mathcal{T}$-open sets or a $\mathcal{T}_{\mathfrak{g}}$-space in terms of a collection of $\mathcal{T}_{\mathfrak{g}}$-open or $\mathfrak{g}-\mathcal{T}_{\mathfrak{g}}$-open sets, respectively, does little to guarantee that the points in the $\mathcal{T}$-space $\mathfrak{T}$ or the $\mathcal{T}_{\mathfrak{g}}$-space $\mathfrak{T}_{\mathfrak{g}}$ are somehow distinct or far apart. The more types and categories of $T_{\alpha}$ or $\mathfrak{g}-\mathrm{T}_{\alpha}$-axioms or $\mathfrak{g}-\mathrm{T}_{\alpha}$ or $\mathfrak{g}-\mathrm{T}_{\mathfrak{g}, \alpha}$-axioms (with $\alpha=0$, $1,2, \ldots)$ are added to the axioms for a $\mathcal{T}$-space or a $\mathcal{T}_{\mathfrak{g}}$-space, respectively, the greater the role they will play in any topological endeavours $[4,8,10,18,23]$. For instance, for a sequence $\left\langle\mathfrak{g}-\mathrm{T}_{\mathfrak{g}, \alpha}, \mathfrak{g}-\mathrm{T}_{\mathfrak{g}, \beta}, \mathfrak{g}-\mathrm{T}_{\mathfrak{g}, \gamma}\right\rangle$ (with $\alpha, \beta, \gamma=0,1,2, \ldots$ ), the $\mathfrak{g}-\mathrm{T}_{\mathfrak{g}, \alpha}, \mathfrak{g}-\mathrm{T}_{\mathfrak{g}, \beta}$ and $\mathfrak{g}-\mathrm{T}_{\mathfrak{g}, \gamma}$-axioms can be arranged in increasing order of strength in the sense that $\mathfrak{g}-\mathrm{T}_{\mathfrak{g}, \gamma}$ implies $\mathfrak{g}-\mathrm{T}_{\mathfrak{g}, \beta}$ and the latter implies $\mathfrak{g}-\mathrm{T}_{\mathfrak{g}, \alpha}$.

In the literature of $\mathcal{T}$-spaces and $\mathcal{T}_{\mathfrak{g}}$-spaces, respectively, several classes of $T_{\alpha}$, $\mathfrak{g}$ - $\mathrm{T}_{\alpha}$-axioms, founded upon the concepts of $\mathcal{T}, \mathfrak{g}$ - $\mathcal{T}$-open sets, and $\mathrm{T}_{\mathfrak{g}, \alpha}, \mathfrak{g}-\mathrm{T}_{\mathfrak{g}, \alpha^{-}}$ axioms (with $\alpha=0,1,2, \ldots$ ), founded upon the concepts of $\mathcal{T}_{\mathfrak{g}}, \mathfrak{g}-\mathcal{T}_{\mathfrak{g}}$-open sets, have been introduced and studied $[1,9,11,12,13,20,22]$. The $\mathrm{T}_{\alpha}$-axioms called $\mathrm{T}_{\text {Kolmogorov }}, \mathrm{T}_{\text {Fréchet }}, \mathrm{T}_{\text {Hausdorff }}, \mathrm{T}_{\text {Regular }}$, and $\mathrm{T}_{\text {Normal-axioms (shortly, }} \mathrm{T}_{\mathrm{K}}, \mathrm{T}_{\mathrm{F}}$, $\mathrm{T}_{\mathrm{H}}, \mathrm{T}_{\mathrm{R}}$, and $\mathrm{T}_{\mathrm{N}}$ ), founded upon the concepts of $\mathcal{T}$-open, closed sets, are four classical examples, among others, which have gained extensive studies [27]. The

\footnotetext{
${ }^{1}$ Notes to the reader: The notations $\mathrm{T}_{\alpha}$-axiom and $\mathfrak{g}$ - $\mathrm{T}_{\alpha}$-axiom (with $\alpha=0,1,2, \ldots$ ), founded upon the notions of $\mathcal{T}$-open and $\mathfrak{g}-\mathcal{T}$-open sets, respectively, designate an ordinary and

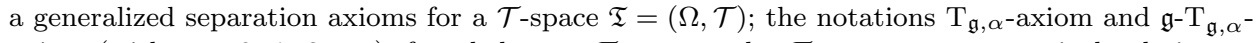
axiom (with $\alpha=0,1,2, \ldots$ ), founded upon $\mathcal{T}_{\mathfrak{g}}$-open and $\mathfrak{g}$ - $\mathcal{T}_{\mathfrak{g}}$-open sets, respectively, designate an ordinary and a generalized separation axioms for a $\mathcal{T}_{\mathfrak{g}}$-space $\mathfrak{T}=\left(\Omega, \mathcal{T}_{\mathfrak{g}}\right)$.
} 
$\mathfrak{g}$ - $\mathrm{T}_{\alpha}$-axioms called generalized $\mathrm{T}_{\alpha}, \mathrm{S}_{\beta}$-axioms (with $\alpha=0,1,2 ; \beta=1,2$ ), founded upon $\mathfrak{g}-\mathcal{T}$-open sets instead of $\mathcal{T}$-open sets, are five examples of generalized $\mathrm{T}_{\alpha^{-}}$ axioms which have been discussed in the paper of [5]; the $\mathfrak{g}-\mathrm{T}_{\mathfrak{g}, \alpha}$-axioms called generalized $\mathrm{T}_{\frac{\alpha}{8}}$-axioms (with $\alpha=2,3,4$ ), founded upon $\mathfrak{g}-\mathcal{T}_{\mathfrak{g}}$-open sets instead of $\mathcal{T}_{\mathfrak{g}}$-open sets, are three examples of generalized $T_{\mathfrak{g}, \alpha}$-axioms which have introduced and studied by [26]. Several other class of $\mathrm{T}_{\alpha}, \mathfrak{g}-\mathrm{T}_{\alpha}$-axioms and $\mathrm{T}_{\mathfrak{g}, \alpha}, \mathfrak{g}$ - $\mathrm{T}_{\mathfrak{g}, \alpha}$-axioms (with $\alpha=0,1,2, \ldots$ ) have also been introduced and discussed in many papers $[14,16,20,25,24,28]$.

In view of the above references, we remark that the quintuple sequence $\left\langle\mathrm{T}_{\alpha}\right\rangle_{\alpha \in \Lambda}$, where $\Lambda=\{\mathrm{K}, \mathrm{F}, \mathrm{H}, \mathrm{R}, \mathrm{N}\}$, is based on the notions of $\mathcal{T}$-open, closed sets. From this remark and the conclusion drawn by [5], it is no error to state that $\left\langle\mathfrak{g}-\mathrm{T}_{\alpha}\right\rangle_{\alpha \in \Lambda}$ are based on the notions of $\mathfrak{g}-\mathcal{T}$-open, closed sets; $\left\langle\mathrm{T}_{\mathfrak{g}, \alpha}\right\rangle_{\alpha \in \Lambda}$ on the notions of $\mathcal{T}_{\mathfrak{g}}$-open, closed sets, and $\left\langle\mathfrak{g}-\mathrm{T}_{\mathfrak{g}, \alpha}\right\rangle_{\alpha \in \Lambda}$ on the notions of $\mathfrak{g}-\mathcal{T}_{\mathfrak{g}}$-open, closed sets. Thus, the idea of adding a quintuple sequence $\left\langle\mathfrak{g}-\mathrm{T}_{\mathfrak{g}, \alpha}\right\rangle_{\alpha \in \Lambda}$ of $\mathfrak{g}-\mathrm{T}_{\mathfrak{g}, \alpha}$-axioms (with $\Lambda=\{\mathrm{K}, \mathrm{F}, \mathrm{H}, \mathrm{R}, \mathrm{N}\}$ ), founded upon a new class of $\mathfrak{g}$ - $\mathcal{T}_{\mathfrak{g}}$-open, closed sets, to the axioms for a $\mathcal{T}_{\mathfrak{g}}$-space $\mathfrak{T}_{\mathfrak{g}}=\left(\Omega, \mathcal{T}_{\mathfrak{g}}\right)$ to obtain a corresponding sequence $\left\langle\mathfrak{g}-\mathfrak{T}_{\mathfrak{g}}^{(\alpha)}=\right.$ $\left.\left(\Omega, \mathfrak{g}-\mathcal{T}_{\mathfrak{g}}{ }^{(\alpha)}\right)\right\rangle_{\alpha \in \Lambda}$ of $\mathfrak{g}-\mathcal{T}_{\mathfrak{g}}^{(\alpha)}$-spaces might be an interesting subject of inquiry.

Hitherto, the introduction of several types of $\mathrm{T}_{\alpha}$ and $\mathfrak{g}$ - $\mathrm{T}_{\alpha}$-axioms in $\mathcal{T}$-spaces and $\mathrm{T}_{\mathfrak{g}, \alpha}$ and $\mathfrak{g}$ - $\mathrm{T}_{\mathfrak{g}, \alpha}$-axioms (with $\alpha=0,1,2, \ldots$ ) in $\mathcal{T}_{\mathfrak{g}}$-spaces have contributed extensively to the geometrical specifications of $\mathcal{T}$-spaces and $\mathcal{T}_{\mathfrak{g}}$-spaces. However, despite these contributions not a single work has been devoted to the generalization of the sequence $\left\langle\mathrm{T}_{\alpha}\right\rangle_{\alpha \in \Lambda}$ in terms of the notions of $\mathfrak{g}$ - $\mathcal{T}$-open, closed sets. With this view in mind, the idea therefore suggests itself, of introducing the generalized versions of the Kolmogorov, Fréchet, Hausdorff, Regular and Normal separation axioms in terms of the notions of $\mathfrak{g}-\mathcal{T}$-open, closed sets in a $\mathcal{T}_{\mathfrak{g}}$-space, adequate for the obtention of $\mathfrak{g}-\mathcal{T}$-spaces in this direction.

In this paper, we attempt to make a contribution to such a development by introducing a new theory, called Theory of $\mathfrak{g}-\mathfrak{T}_{\mathfrak{g}}$-Separation Axioms, in which it is presented the generalized version of the sequence $\left\langle\mathrm{T}_{\alpha}\right\rangle_{\alpha \in \Lambda}$ in terms of the notions of $\mathfrak{g}-\mathcal{T}$-open, closed sets, discussing the fundamental properties and giving characterizations of its elements, on this ground and with respect to existing works.

The paper is organised as follows: In SECT. 2, preliminary notions are described in SECT. 2.1 and the main results of the $\mathfrak{g}$ - $\mathrm{T}_{\mathfrak{g}, \alpha}$-axioms in a $\mathcal{T}_{\mathfrak{g}}$-space are reported in SECt. 3. In SECT. 4, the establishment of the various relationships between these $\mathfrak{g}-\mathrm{T}_{\mathfrak{g}, \alpha^{-a x i o m s}}$ are discussed in SECT. 4.1. To support the work, a nice application of the $\mathfrak{g}$-T $\mathrm{T}_{\mathfrak{g}, \alpha}$-axioms in a $\mathcal{T}_{\mathfrak{g}}$-space is presented in SECT. 4.2. Finally, SECT. 4.3 provides concluding remarks and future directions of the $\mathfrak{g}-\mathrm{T}_{\mathfrak{g}, \alpha}$-axioms in a $\mathcal{T}_{\mathfrak{g}}$-space.

\section{THEORY}

2.1. Preliminaries. Though foreign terms are neatly defined in complementary chapters (see our papers on theories of $\mathfrak{g}-\mathfrak{T}_{\mathfrak{g}}$-sets and $\mathfrak{g}$ - $\mathfrak{T}_{\mathfrak{g}}$-maps), we thought it necessary to recall some basic definitions and notations of most essential concepts presented in those chapters.

The set $\mathfrak{U}$ represents the universe of discourse, fixed within the framework of the theory of $\mathfrak{g}-\mathfrak{T}_{\mathfrak{g}}$-separation axioms and containing as elements all sets $(\mathcal{T}, \mathfrak{g}-\mathcal{T}, \mathfrak{T}$, 
$\mathfrak{g}$-T-sets; $\mathcal{T}_{\mathfrak{g}}, \mathfrak{g}-\mathcal{T}_{\mathfrak{g}}, \mathfrak{T}_{\mathfrak{g}}, \mathfrak{g}$ - $\mathfrak{T}_{\mathfrak{g}}$-sets $)$ considered in this theory, and $I_{n}^{0} \stackrel{\text { def }}{=}\left\{\nu \in \mathbb{N}^{0}\right.$ : $\nu \leq n\}$; index sets $I_{\infty}^{0}, I_{n}^{*}, I_{\infty}^{*}$ are defined in an analogous way. Granted $\Omega \subset \mathfrak{U}$, $\mathcal{P}(\Omega) \stackrel{\text { def }}{=}\left\{\mathcal{O}_{\mathfrak{g}, \nu} \subseteq \Omega: \nu \in I_{\infty}^{*}\right\}$ denotes the family of all subsets $\mathcal{O}_{\mathfrak{g}, 1}, \mathcal{O}_{\mathfrak{g}, 2}, \ldots$, of $\Omega$. A one-valued map of the type $\mathcal{T}_{\mathfrak{g}}: \mathcal{P}(\Omega) \rightarrow \mathcal{P}(\Omega)$ satisfying $\mathcal{T}_{\mathfrak{g}}(\emptyset)=\emptyset$, $\mathcal{T}_{\mathfrak{g}}\left(\mathcal{O}_{\mathfrak{g}}\right) \subseteq \mathcal{O}_{\mathfrak{g}}$, and $\mathcal{T}_{\mathfrak{g}}\left(\bigcup_{\nu \in I_{\infty}^{*}} \mathcal{O}_{\mathfrak{g}, \nu}\right)=\bigcup_{\nu \in I_{\infty}^{*}} \mathcal{T}_{\mathfrak{g}}\left(\mathcal{O}_{\mathfrak{g}, \nu}\right)$ is called a $\mathfrak{g}$-topology on $\Omega$, and the structure $\mathfrak{T}_{\mathfrak{g}} \stackrel{\text { def }}{=}\left(\Omega, \mathcal{T}_{\mathfrak{g}}\right)$ is called a $\mathcal{T}_{\mathfrak{g}}$-space, on which a quintuple sequence $\left\langle\mathfrak{g}-\mathrm{T}_{\mathfrak{g}, \alpha}\right\rangle_{\alpha \in \Lambda}$ of $\mathfrak{g}-\mathrm{T}_{\mathfrak{g}, \alpha}$-axioms (with $\Lambda=\{\mathrm{K}, \mathrm{F}, \mathrm{H}, \mathrm{R}, \mathrm{N}\}$ ) will be discussed [7, 6, 21].

The operator cl $\mathrm{l}_{\mathfrak{g}}: \mathcal{P}(\Omega) \rightarrow \mathcal{P}(\Omega)$ carrying each $\mathfrak{T}_{\mathfrak{g}}$-set $\mathcal{S}_{\mathfrak{g}} \subset \mathfrak{T}_{\mathfrak{g}, \Omega}$ into its closure $\operatorname{cl}_{\mathfrak{g}}\left(\mathcal{S}_{\mathfrak{g}}\right)=\mathfrak{T}_{\mathfrak{g}}-\operatorname{int}_{\mathfrak{g}}\left(\mathfrak{T}_{\mathfrak{g}} \backslash \mathcal{S}_{\mathfrak{g}}\right) \subset \mathfrak{T}_{\mathfrak{g}}$ is termed a $\mathfrak{g}$-closure operator and the operator $\operatorname{int}_{\mathfrak{g}}: \mathcal{P}(\Omega) \rightarrow \mathcal{P}(\Omega)$ carrying each $\mathfrak{T}_{\mathfrak{g}}$-set $\mathcal{S}_{\mathfrak{g}} \subset \mathfrak{T}_{\mathfrak{g}}$ into its interior $\operatorname{int}_{\mathfrak{g}}\left(\mathcal{S}_{\mathfrak{g}}\right)=$ $\mathfrak{T}_{\mathfrak{g}}-\operatorname{cl}_{\mathfrak{g}}\left(\mathfrak{T}_{\mathfrak{g}} \backslash \mathcal{S}_{\mathfrak{g}}\right) \subset \mathfrak{T}_{\mathfrak{g}}$ is called a $\mathfrak{g}$-interior operator. Let $\complement: \mathcal{P}(\Omega) \rightarrow \mathcal{P}(\Omega)$ denotes the absolute complement with respect to the underlying set $\Omega \subset \mathfrak{U}$, and let $\mathcal{S}_{\mathfrak{g}} \subset \mathfrak{T}_{\mathfrak{g}}$ be any $\mathfrak{T}_{\mathfrak{g}}$-set. The classes

$$
\begin{aligned}
\mathcal{T}_{\mathfrak{g}} & \stackrel{\text { def }}{=}\left\{\mathcal{O}_{\mathfrak{g}} \subset \mathfrak{T}_{\mathfrak{g}}: \mathcal{O}_{\mathfrak{g}} \in \mathcal{T}_{\mathfrak{g}}\right\}, \\
\neg \mathcal{T}_{\mathfrak{g}} & \stackrel{\text { def }}{=}\left\{\mathcal{K}_{\mathfrak{g}} \subset \mathfrak{T}_{\mathfrak{g}}: \complement_{\Lambda}\left(\mathcal{K}_{\mathfrak{g}}\right) \in \mathcal{T}_{\mathfrak{g}}\right\},
\end{aligned}
$$

respectively, denote the classes of all $\mathcal{T}_{\mathfrak{g}}$-open and $\mathcal{T}_{\mathfrak{g}}$-closed sets relative to the $\mathfrak{g}$-topology $\mathcal{T}_{\mathfrak{g}}$, and the classes

$$
\begin{aligned}
& \mathrm{C}_{\mathcal{T}_{\mathfrak{g}}}^{\text {sub }}\left[\mathcal{S}_{\mathfrak{g}}\right] \stackrel{\text { def }}{=}\left\{\mathcal{O}_{\mathfrak{g}} \in \mathcal{T}_{\mathfrak{g}}: \mathcal{O}_{\mathfrak{g}} \subseteq \mathcal{S}_{\mathfrak{g}}\right\}, \\
& \mathrm{C}_{\neg \mathcal{T}_{\mathfrak{g}}}^{\text {sup }},\left[\mathcal{S}_{\mathfrak{g}}\right] \stackrel{\text { def }}{=}\left\{\mathcal{K}_{\mathfrak{g}} \in \neg \mathcal{T}_{\mathfrak{g}}: \mathcal{K}_{\mathfrak{g}} \supseteq \mathcal{S}_{\mathfrak{g}}\right\},
\end{aligned}
$$

respectively, denote the classes of $\mathcal{T}_{\mathfrak{g}}$-open subsets and $\mathcal{T}_{\mathfrak{g}}$-closed supersets (complements of the $\mathcal{T}_{\mathfrak{g}}$-open subsets) of the $\mathfrak{T}_{\mathfrak{g}}$-set $\mathcal{S}_{\mathfrak{g}} \subset \mathfrak{T}_{\mathfrak{g}}$ relative to the $\mathfrak{g}$-topology $\mathcal{T}_{\mathfrak{g}}$. To this end, the $\mathfrak{g}$-closure and the $\mathfrak{g}$-interior of a $\mathfrak{T}_{\mathfrak{g}}$-set $\mathcal{S}_{\mathfrak{g}} \subset \mathfrak{T}_{\mathfrak{g}}$ in a $\mathcal{T}_{\mathfrak{g}}$-space [2] define themselves as

$$
\operatorname{int}_{\mathfrak{g}}\left(\mathcal{S}_{\mathfrak{g}}\right) \stackrel{\text { def }}{=} \bigcup_{\mathcal{O}_{\mathfrak{g}} \in \mathrm{C}_{\mathcal{T}_{\mathfrak{g}}}^{\text {sub }}\left[\mathcal{S}_{\mathfrak{g}}\right]} \mathcal{O}_{\mathfrak{g}}, \quad \operatorname{cl}_{\mathfrak{g}}\left(\mathcal{S}_{\mathfrak{g}}\right) \stackrel{\text { def }}{=} \bigcap_{\mathcal{K}_{\mathfrak{g}} \in \mathrm{C}_{\neg \mathcal{T}_{\mathfrak{g}}}^{\text {sup }}\left[\mathcal{S}_{\mathfrak{g}}\right]} \mathcal{K}_{\mathfrak{g}} .
$$

Throughout this paper, the composition operators $\operatorname{cl}_{\mathfrak{g}} \circ \operatorname{int}_{\mathfrak{g}}(\cdot), \operatorname{int}_{\mathfrak{g}} \circ \mathrm{cl}_{\mathfrak{g}}(\cdot)$, and $\mathrm{cl}_{\mathfrak{g}} \circ \operatorname{int}_{\mathfrak{g}} \circ \mathrm{cl}_{\mathfrak{g}}(\cdot)$, respectively, stand for the functionals $\mathrm{cl}_{\mathfrak{g}}\left(\operatorname{int}_{\mathfrak{g}}(\cdot)\right)$, $\operatorname{int}_{\mathfrak{g}}\left(\mathrm{cl}_{\mathfrak{g}}(\cdot)\right)$, and $\operatorname{cl}_{\mathfrak{g}}\left(\operatorname{int}_{\mathfrak{g}}\left(\mathrm{cl}_{\mathfrak{g}}(\cdot)\right)\right)$; other composition operators are defined similarly. Furthermore, the backslash $\mathfrak{T}_{\mathfrak{g}} \backslash \mathcal{S}_{\mathfrak{g}}$ refers to the set-theoretic difference $\mathfrak{T}_{\mathfrak{g}}-\mathcal{S}_{\mathfrak{g}}$. The mapping op $\mathfrak{g}_{\mathfrak{g}}: \mathcal{P}(\Omega) \rightarrow \mathcal{P}(\Omega)$ is called a $\mathfrak{g}$-operation on $\mathcal{P}(\Omega)$ if the following statements hold:

$$
\begin{aligned}
& \forall \mathcal{S}_{\mathfrak{g}} \in \mathcal{P}(\Omega) \backslash\{\emptyset\}, \exists\left(\mathcal{O}_{\mathfrak{g}}, \mathcal{K}_{\mathfrak{g}}\right) \in \mathcal{T}_{\mathfrak{g}} \backslash\{\emptyset\} \times \neg \mathcal{T}_{\mathfrak{g}} \backslash\{\emptyset\}: \\
& \left(\operatorname{op}_{\mathfrak{g}}(\emptyset)=\emptyset\right) \vee\left(\neg \mathrm{op}_{\mathfrak{g}}(\emptyset)=\emptyset\right),\left(\mathcal{S}_{\mathfrak{g}} \subseteq \operatorname{op}_{\mathfrak{g}}\left(\mathcal{O}_{\mathfrak{g}}\right)\right) \vee\left(\mathcal{S}_{\mathfrak{g}} \supseteq \neg \mathrm{op}_{\mathfrak{g}}\left(\mathcal{K}_{\mathfrak{g}}\right)\right),
\end{aligned}
$$

where $\neg \mathrm{op}_{\mathfrak{g}}: \mathcal{P}(\Omega) \rightarrow \mathcal{P}(\Omega)$ is called the "complementary $\mathfrak{g}$-operation" on $\mathcal{P}(\Omega)$ and, for all $\mathfrak{T}_{\mathfrak{g}}$-sets $\mathcal{S}_{\mathfrak{g}}, \mathcal{S}_{\mathfrak{g}, \nu}, \mathcal{S}_{\mathfrak{g}, \mu} \in \mathcal{P}(\Omega) \backslash\{\emptyset\}$, the following axioms are satisfied:

- Ax. I. $\left(\mathcal{S}_{\mathfrak{g}} \subseteq \mathrm{op}_{\mathfrak{g}}\left(\mathcal{O}_{\mathfrak{g}}\right)\right) \vee\left(\mathcal{S}_{\mathfrak{g}} \supseteq \neg \mathrm{op}_{\mathfrak{g}}\left(\mathcal{K}_{\mathfrak{g}}\right)\right)$,

- Ax. II. $\left(\operatorname{op}_{\mathfrak{g}}\left(\mathcal{S}_{\mathfrak{g}}\right) \subseteq \mathrm{op}_{\mathfrak{g}} \circ \mathrm{op}_{\mathfrak{g}}\left(\mathcal{O}_{\mathfrak{g}}\right)\right) \vee\left(\neg \mathrm{op}_{\mathfrak{g}}\left(\mathcal{S}_{\mathfrak{g}}\right) \supseteq \neg \mathrm{op}_{\mathfrak{g}} \circ \neg \mathrm{op}_{\mathfrak{g}}\left(\mathcal{K}_{\mathfrak{g}}\right)\right)$,

- Ax. III. $\left(\mathcal{S}_{\mathfrak{g}, \nu} \subseteq \mathcal{S}_{\mathfrak{g}, \mu} \rightarrow \mathrm{op}_{\mathfrak{g}}\left(\mathcal{O}_{\mathfrak{g}, \nu}\right) \subseteq \mathrm{op}_{\mathfrak{g}}\left(\mathcal{O}_{\mathfrak{g}, \mu}\right)\right) \vee\left(\mathcal{S}_{\mathfrak{g}, \mu} \subseteq \mathcal{S}_{\mathfrak{g}, \nu} \leftarrow\right.$ $\left.\neg \mathrm{op}_{\mathfrak{g}}\left(\mathcal{K}_{\mathfrak{g}, \mu}\right) \supseteq \neg \mathrm{op}_{\mathfrak{g}}\left(\mathcal{K}_{\mathfrak{g}, \nu}\right)\right)$, 
- Ax. IV. $\left(\operatorname{op}_{\mathfrak{g}}\left(\bigcup_{\sigma=\nu, \mu} \mathcal{S}_{\mathfrak{g}, \sigma}\right) \subseteq \bigcup_{\sigma=\nu, \mu} \mathrm{op}_{\mathfrak{g}}\left(\mathcal{O}_{\mathfrak{g}, \sigma}\right)\right) \vee\left(\neg \mathrm{op}_{\mathfrak{g}}\left(\bigcup_{\sigma=\nu, \mu} \mathcal{S}_{\mathfrak{g}, \sigma}\right) \supseteq\right.$ $\left.\bigcup_{\sigma=\nu, \mu} \neg \mathrm{op}_{\mathfrak{g}}\left(\mathcal{K}_{\mathfrak{g}, \sigma}\right)\right)$,

for some $\mathcal{T}_{\mathfrak{g}}$-open sets $\mathcal{O}_{\mathfrak{g}}, \mathcal{O}_{\mathfrak{g}, \nu}, \mathcal{O}_{\mathfrak{g}, \mu} \in \mathcal{T}_{\mathfrak{g}} \backslash\{\emptyset\}$ and $\mathcal{T}_{\mathfrak{g}}$-closed sets $\mathcal{K}_{\mathfrak{g}}, \mathcal{K}_{\mathfrak{g}, \nu}$, $\mathcal{K}_{\mathfrak{g}, \mu} \in \neg \mathcal{T}_{\mathfrak{g}}[3,15]$. The class $\mathcal{L}_{\mathfrak{g}}[\Omega]=\mathcal{L}_{\mathfrak{g}}^{\omega}[\Omega] \times \mathcal{L}_{\mathfrak{g}}^{\kappa}[\Omega]$, where

$$
\mathcal{L}_{\mathfrak{g}}[\Omega] \stackrel{\text { def }}{=}\left\{\mathbf{o p}_{\mathfrak{g}, \nu \mu}(\cdot)=\left(\mathrm{op}_{\mathfrak{g}, \nu}(\cdot), \neg \mathrm{op}_{\mathfrak{g}, \mu}(\cdot)\right):(\nu, \mu) \in I_{3}^{0} \times I_{3}^{0}\right\}
$$

in the $\mathcal{T}_{\mathfrak{g}}$-space $\mathfrak{T}_{\mathfrak{g}}$, stands for the class of all possible $\mathfrak{g}$-operators and their complementary $\mathfrak{g}$-operators in the $\mathcal{T}_{\mathfrak{g}}$-space $\mathfrak{T}_{\mathfrak{g}}$. Its elements are defined as:

$$
\begin{aligned}
\operatorname{op}_{\mathfrak{g}}(\cdot) & \in \mathcal{L}_{\mathfrak{g}}^{\omega}[\Omega] \stackrel{\text { def }}{=}\left\{\operatorname{op}_{\mathfrak{g}, 0}(\cdot), \operatorname{op}_{\mathfrak{g}, 1}(\cdot), \operatorname{op}_{\mathfrak{g}, 2}(\cdot), \operatorname{op}_{\mathfrak{g}, 3}(\cdot)\right\} \\
& =\left\{\operatorname{int}_{\mathfrak{g}}(\cdot), \operatorname{cl}_{\mathfrak{g}} \circ \operatorname{int}_{\mathfrak{g}}(\cdot), \operatorname{int}_{\mathfrak{g}} \circ \operatorname{cl}_{\mathfrak{g}}(\cdot), \operatorname{cl}_{\mathfrak{g}} \circ \operatorname{int}_{\mathfrak{g}} \circ \mathrm{cl}_{\mathfrak{g}}(\cdot)\right\} ; \\
\neg \mathrm{op}_{\mathfrak{g}}(\cdot) & \in \mathcal{L}_{\mathfrak{g}}^{\kappa}[\Omega] \stackrel{\operatorname{def}^{=}}{=}\left\{\neg \operatorname{op}_{\mathfrak{g}, 0}(\cdot), \neg \operatorname{op}_{\mathfrak{g}, 1}(\cdot), \neg \operatorname{op}_{\mathfrak{g}, 2}(\cdot), \neg \mathrm{op}_{\mathfrak{g}, 3}(\cdot)\right\} \\
& =\left\{\operatorname{cl}_{\mathfrak{g}}(\cdot), \operatorname{int}_{\mathfrak{g}} \circ \mathrm{cl}_{\mathfrak{g}}(\cdot), \operatorname{cl}_{\mathfrak{g}} \circ \operatorname{int}_{\mathfrak{g}}(\cdot), \operatorname{int}_{\mathfrak{g}} \circ \operatorname{cl}_{\mathfrak{g}} \circ \operatorname{int}_{\mathfrak{g}}(\cdot)\right\} .
\end{aligned}
$$

A $\mathfrak{T}_{\mathfrak{g}}$-set $\mathcal{S}_{\mathfrak{g}} \subset \mathfrak{T}_{\mathfrak{g}}$ in a $\mathcal{T}_{\mathfrak{g}}$-space is called a $\mathfrak{g}$ - $\mathfrak{T}_{\mathfrak{g}}$-set if and only if there exist a pair $\left(\mathcal{O}_{\mathfrak{g}}, \mathcal{K}_{\mathfrak{g}}\right) \in \mathcal{T}_{\mathfrak{g}} \times \neg \mathcal{T}_{\mathfrak{g}}$ of $\mathcal{T}_{\mathfrak{g}}$-open and $\mathcal{T}_{\mathfrak{g}}$-closed sets, and a $\mathfrak{g}$-operator $\mathbf{o p}_{\mathfrak{g}}(\cdot) \in \mathcal{L}_{\mathfrak{g}}[\Omega]$ such that the following statement holds:

$$
(\exists \xi)\left[\left(\xi \in \mathcal{S}_{\mathfrak{g}}\right) \wedge\left(\left(\mathcal{S}_{\mathfrak{g}} \subseteq \mathrm{op}_{\mathfrak{g}}\left(\mathcal{O}_{\mathfrak{g}}\right)\right) \vee\left(\mathcal{S}_{\mathfrak{g}} \supseteq \neg \mathrm{op}_{\mathfrak{g}}\left(\mathcal{K}_{\mathfrak{g}}\right)\right)\right)\right] .
$$

The $\mathfrak{g}$ - $\mathfrak{T}_{\mathfrak{g}}$-set $\mathcal{S}_{\mathfrak{g}} \subset \mathfrak{T}_{\mathfrak{g}}$ is said to be of category $\nu$ if and only if it belongs to the following class of $\mathfrak{g}-\nu$ - $\mathfrak{T}_{\mathfrak{g}}$-sets:

$$
\begin{aligned}
\mathfrak{g}-\nu \text {-S }\left[\mathfrak{T}_{\mathfrak{g}}\right] \stackrel{\text { def }}{=} & \left\{\mathcal{S}_{\mathfrak{g}} \subset \mathfrak{T}_{\mathfrak{g}}:\left(\exists \mathcal{O}_{\mathfrak{g}}, \mathcal{K}_{\mathfrak{g}}, \mathbf{o p}_{\mathfrak{g}, \nu}(\cdot)\right)\right. \\
& {\left.\left[\left(\mathcal{S}_{\mathfrak{g}} \subseteq \mathrm{op}_{\mathfrak{g}, \nu}\left(\mathcal{O}_{\mathfrak{g}}\right)\right) \vee\left(\mathcal{S}_{\mathfrak{g}} \supseteq \neg \mathrm{op}_{\mathfrak{g}, \nu}\left(\mathcal{K}_{\mathfrak{g}}\right)\right)\right]\right\} . }
\end{aligned}
$$

It is called a $\mathfrak{g}-\nu-\mathfrak{T}_{\mathfrak{g}}$-open set if it satisfies the first property in $\mathfrak{g}-\nu$-S $\left[\mathfrak{T}_{\mathfrak{g}}\right]$ and a $\mathfrak{g}-\nu-\mathfrak{T}_{\mathfrak{g}}$-closed set if it satisfies the second property in $\mathfrak{g}-\nu-\mathrm{S}\left[\mathfrak{T}_{\mathfrak{g}}\right]$. The classes of $\mathfrak{g}-\nu-\mathfrak{T}_{\mathfrak{g}}$-open and $\mathfrak{g}-\nu-\mathfrak{T}_{\mathfrak{g}}$-closed sets, respectively, are defined by

$$
\begin{aligned}
& \mathfrak{g}-\nu-\mathrm{O}\left[\mathfrak{T}_{\mathfrak{g}}\right] \stackrel{\text { def }}{=}\left\{\mathcal{S}_{\mathfrak{g}} \subset \mathfrak{T}_{\mathfrak{g}}:\left(\exists \mathcal{O}_{\mathfrak{g}}, \mathbf{o p}_{\mathfrak{g}, \nu}(\cdot)\right)\left[\mathcal{S}_{\mathfrak{g}} \subseteq \mathrm{op}_{\mathfrak{g}, \nu}\left(\mathcal{O}_{\mathfrak{g}}\right)\right]\right\}, \\
& \mathfrak{g}-\nu-\mathrm{K}\left[\mathfrak{T}_{\mathfrak{g}}\right] \stackrel{\text { def }}{=}\left\{\mathcal{S}_{\mathfrak{g}} \subset \mathfrak{T}_{\mathfrak{g}}:\left(\exists \mathcal{K}_{\mathfrak{g}}, \mathbf{o p}_{\mathfrak{g}, \nu}(\cdot)\right)\left[\mathcal{S}_{\mathfrak{g}} \supseteq \neg \mathrm{op}_{\mathfrak{g}, \nu}\left(\mathcal{K}_{\mathfrak{g}}\right)\right]\right\} .
\end{aligned}
$$

From these classes, the following relation holds:

$$
\begin{aligned}
\mathfrak{g}-\mathrm{S}\left[\mathfrak{T}_{\mathfrak{g}}\right] & =\bigcup_{\nu \in I_{3}^{0}} \mathfrak{g}-\nu-\mathrm{S}\left[\mathfrak{T}_{\mathfrak{g}}\right] \\
& =\bigcup_{\nu \in I_{3}^{0}}\left(\mathfrak{g}-\nu-\mathrm{O}\left[\mathfrak{T}_{\mathfrak{g}}\right] \cup \mathfrak{g}-\nu-\mathrm{K}\left[\mathfrak{T}_{\mathfrak{g}}\right]\right) \\
& =\left(\bigcup_{\nu \in I_{3}^{0}} \mathfrak{g}-\nu-\mathrm{O}\left[\mathfrak{T}_{\mathfrak{g}}\right]\right) \cup\left(\bigcup_{\nu \in I_{3}^{0}} \mathfrak{g}-\nu-\mathrm{K}\left[\mathfrak{T}_{\mathfrak{g}}\right]\right) \\
& =\mathfrak{g}-\mathrm{O}\left[\mathfrak{T}_{\mathfrak{g}}\right] \cup \mathfrak{g}-\mathrm{K}\left[\mathfrak{T}_{\mathfrak{g}}\right] .
\end{aligned}
$$

When the subscript $\mathfrak{g}$ are omitted in almost all symbols of the above definitions, very similar definitions are derived but in a $\mathcal{T}$-space.

A $\mathfrak{T}$-set $\mathcal{S} \subset \mathfrak{T}$ in a $\mathcal{T}$-space is called a $\mathfrak{g}$-T $\mathfrak{T}$-set if and only if there exists a pair $(\mathcal{O}, \mathcal{K}) \in \mathcal{T} \times \neg \mathcal{T}$ of $\mathcal{T}$-open and $\mathcal{T}$-closed sets, and an operator op $(\cdot) \in \mathcal{L}[\Omega]$ such that the following statement holds:

$$
(\exists \xi)[(\xi \in \mathcal{S}) \wedge((\mathcal{S} \subseteq \text { op }(\mathcal{O})) \vee(\mathcal{S} \supseteq \neg \text { op }(\mathcal{K})))] .
$$


The $\mathfrak{g}$-T -set $\mathcal{S} \subset \mathfrak{T}$ is said to be of category $\nu$ if and only if it belongs to the following class of $\mathfrak{g}-\nu$ - $\mathcal{T}$-sets:

$$
\begin{aligned}
\mathfrak{g}-\nu \text {-S }[\mathfrak{T}] \stackrel{\text { def }}{=}\left\{\mathcal{S} \subset \mathfrak{T}:\left(\exists \mathcal{O}, \mathcal{K}, \mathbf{o p}_{\nu}(\cdot)\right)\right. \\
\\
{\left.\left[\left(\mathcal{S} \subseteq \mathrm{op}_{\nu}(\mathcal{O})\right) \vee\left(\mathcal{S} \supseteq \neg \mathrm{op}_{\nu}(\mathcal{K})\right)\right]\right\} . }
\end{aligned}
$$

It is called a $\mathfrak{g}-\nu$ - $\mathfrak{T}$-open set if it satisfies the first property in $\mathfrak{g}-\nu$-S $[\mathfrak{T}]$ and a $\mathfrak{g}-\nu$ - $\mathfrak{T}$ closed set if it satisfies the second property in $\mathfrak{g}-\nu$-S $[\mathfrak{T}]$. The classes of $\mathfrak{g}-\nu$ - $\mathfrak{T}$-open and $\mathfrak{g}-\nu$-T-closed sets, respectively, are defined by

$$
\begin{aligned}
& \mathfrak{g}-\nu-\mathrm{O}[\mathfrak{T}] \stackrel{\text { def }}{=}\left\{\mathcal{S} \subset \mathfrak{T}:\left(\exists \mathcal{O}, \mathbf{o p}_{\nu}(\cdot)\right)\left[\mathcal{S} \subseteq \mathrm{op}_{\nu}(\mathcal{O})\right]\right\}, \\
& \mathfrak{g}-\nu-\mathrm{K}[\mathfrak{T}] \stackrel{\text { def }}{=}\left\{\mathcal{S} \subset \mathfrak{T}:\left(\exists \mathcal{K}, \mathbf{o p}_{\nu}(\cdot)\right)\left[\mathcal{S} \supseteq \neg \mathrm{op}_{\nu}(\mathcal{K})\right]\right\} .
\end{aligned}
$$

The following relations are immediate consequences of the above definitions:

$$
\begin{aligned}
\mathfrak{g}-\mathrm{S}[\mathfrak{T}] & =\bigcup_{\nu \in I_{3}^{0}} \mathfrak{g}-\nu-\mathrm{S}[\mathfrak{T}] \\
& =\bigcup_{\nu \in I_{3}^{0}}(\mathfrak{g}-\nu-\mathrm{O}[\mathfrak{T}] \cup \mathfrak{g}-\nu-\mathrm{K}[\mathfrak{T}]) \\
& =\left(\bigcup_{\nu \in I_{3}^{0}} \mathfrak{g}-\nu-\mathrm{O}[\mathfrak{T}]\right) \cup\left(\bigcup_{\nu \in I_{3}^{0}} \mathfrak{g}-\nu-\mathrm{K}[\mathfrak{T}]\right) \\
& =\mathfrak{g}-\mathrm{O}[\mathfrak{T}] \cup \mathfrak{g}-\mathrm{K}[\mathfrak{T}] .
\end{aligned}
$$

The classes $\mathrm{O}\left[\mathfrak{T}_{\mathfrak{g}}\right]$ and $\mathrm{K}\left[\mathfrak{T}_{\mathfrak{g}}\right]$ denote the families of $\mathfrak{T}_{\mathfrak{g}}$-open and $\mathfrak{T}_{\mathfrak{g}}$-closed sets, respectively, in $\mathfrak{T}_{\mathfrak{g}}$, with $\mathrm{S}\left[\mathfrak{T}_{\mathfrak{g}}\right]=\mathrm{O}\left[\mathfrak{T}_{\mathfrak{g}}\right] \cup \mathrm{K}\left[\mathfrak{T}_{\mathfrak{g}}\right]$; the classes $\mathrm{O}[\mathfrak{T}]$ and $\mathrm{K}[\mathfrak{T}]$ denote the families of $\mathfrak{T}$-open and $\mathfrak{T}$-closed sets, respectively, in $\mathfrak{T}$, with $\mathrm{S}[\mathfrak{T}]=\mathrm{O}[\mathfrak{T}] \cup \mathrm{K}[\mathfrak{T}]$.

In regard to the above descriptions, by a $\mathfrak{g}-\mathcal{T}_{\mathfrak{g}}$-open set and a $\mathfrak{g}-\mathcal{T}_{\mathfrak{g}}$-closed set are meant a $\mathcal{T}_{\mathfrak{g}}$-open set $\mathcal{O}_{\mathfrak{g}} \in \mathcal{T}_{\mathfrak{g}}$ and a $\mathcal{T}_{\mathfrak{g}}$-closed set $\mathcal{K}_{\mathfrak{g}} \in \neg \mathcal{T}_{\mathfrak{g}}$ satisfying $\mathcal{O}_{\mathfrak{g}} \subseteq$ op $\mathfrak{g}_{\mathfrak{g}}\left(\mathcal{O}_{\mathfrak{g}}\right)$ and $\mathcal{K}_{\mathfrak{g}} \supseteq \neg \mathrm{op}_{\mathfrak{g}}\left(\mathcal{K}_{\mathfrak{g}}\right)$, respectively. Likewise, by a $\mathfrak{g}-\mathcal{T}_{\mathfrak{g}}$-open set of category $\nu$ and a $\mathfrak{g}-\mathcal{T}_{\mathfrak{g}}$-closed set of category $\nu$ are meant a $\mathcal{T}_{\mathfrak{g}}$-open set $\mathcal{O}_{\mathfrak{g}} \in \mathcal{T}_{\mathfrak{g}}$ and a $\mathcal{T}_{\mathfrak{g}}$-closed set $\mathcal{K}_{\mathfrak{g}} \in \neg \mathcal{T}_{\mathfrak{g}}$ satisfying $\mathcal{O}_{\mathfrak{g}} \subseteq \mathrm{op}_{\mathfrak{g}, \nu}\left(\mathcal{O}_{\mathfrak{g}}\right)$ and $\mathcal{K}_{\mathfrak{g}} \supseteq \neg \mathrm{op}_{\mathfrak{g}, \nu}\left(\mathcal{K}_{\mathfrak{g}}\right)$, respectively; $\mathfrak{g}-\mathcal{T}_{\mathfrak{g}}-$ sets of category $\nu$ will be called $\mathfrak{g}-\nu-\mathcal{T}_{\mathfrak{g}}$-sets. We are now in a position to present a carefully chosen set of terms used in the theory of $\mathfrak{g}$ - $\mathfrak{T}_{\mathfrak{g}}$-separation axioms in $\mathcal{T}_{\mathfrak{g}}$-spaces.

Agreed to let $\left\langle\mathfrak{g}-\mathrm{T}_{\mathfrak{g}, \alpha}\right\rangle_{\alpha \in \Lambda}$ denote a sequence of $\mathfrak{g}-\mathrm{T}_{\mathfrak{g}, \alpha}$-axioms, indexed by the set $\Lambda \stackrel{\text { def }}{=}\{\mathrm{K}, \mathrm{F}, \mathrm{H}, \mathrm{R}, \mathrm{N}\}$, throughout the present chapter, the sequence $\left\langle\mathfrak{g}-\mathfrak{T}_{\mathfrak{g}}^{(\alpha)}=\right.$ $\left.\left(\Omega, \mathfrak{g}-\mathcal{T}_{\mathfrak{g}}^{(\alpha)}\right)\right\rangle_{\alpha \in \Lambda}$ will stand for the resulting sequence of $\mathfrak{g}-\mathcal{T}_{\mathfrak{g}}^{(\alpha)}$-spaces, obtained after endowing a $\mathcal{T}_{\mathfrak{g}}$-space $\mathfrak{T}_{\mathfrak{g}}=\left(\Omega, \mathcal{T}_{\mathfrak{g}}\right)$ with $\left\langle\mathfrak{g}-\mathrm{T}_{\mathfrak{g}, \alpha}\right\rangle_{\alpha \in \Lambda}$. Hence, the definition follows.

Definition 2.1 $\left(\mathfrak{g}-\mathcal{T}_{\mathfrak{g}}^{(\alpha)}\right.$-Space). A $\mathcal{T}_{\mathfrak{g}}$-space $\mathfrak{T}_{\mathfrak{g}}=\left(\Omega, \mathcal{T}_{\mathfrak{g}}\right)$ endowed with a $\mathfrak{g}$ - $T_{\mathfrak{g}, \alpha^{-}}$ axiom is called a $\mathfrak{g}-\mathcal{T}_{\mathfrak{g}}^{(\alpha)}$-space $\mathfrak{g}-\mathfrak{T}_{\mathfrak{g}}^{(\alpha)} \stackrel{\text { def }}{=}\left(\Omega, \mathfrak{g}-\mathcal{T}_{\mathfrak{g}}^{(\alpha)}\right)$.

The elements of $\left\langle\mathfrak{g}-T_{\mathfrak{g}, \alpha}\right\rangle_{\alpha \in \Lambda}$ concern the separation of points, points from $\mathfrak{g}-\mathcal{T}_{\mathfrak{g}^{-}}$ open sets, and $\mathfrak{g}-\mathcal{T}_{\mathfrak{g}}$-open sets from each other. They are nicely discussed through the notions of pairwise disjoint points and $\mathfrak{g}$ - $\mathcal{T}_{\mathfrak{g}}$-sets in a $\mathcal{T}_{\mathfrak{g}}$-space $\mathfrak{T}_{\mathfrak{g}}$. We let $\left(\mathcal{O}_{\mathfrak{g}, \xi}, \mathcal{K}_{\mathfrak{g}, \xi}\right) \in \mathcal{T}_{\mathfrak{g}} \times \neg \mathcal{T}_{\mathfrak{g}}$ denote a pair of $\mathcal{T}_{\mathfrak{g}}$-open and $\mathcal{T}_{\mathfrak{g}}$-closed sets containing the point $\xi \in \mathfrak{T}_{\mathfrak{g}}$ and let $\left(\mathcal{O}_{\mathfrak{g}, \mathcal{S}_{\mathfrak{g}}}, \mathcal{K}_{\mathfrak{g}, \mathcal{S}_{\mathfrak{g}}}\right) \in \mathcal{T}_{\mathfrak{g}} \times \neg \mathcal{T}_{\mathfrak{g}}$ denote either a pair of $\mathcal{T}_{\mathfrak{g}}$-open and $\mathcal{T}_{\mathfrak{g}}$-closed subsets or supersets of the set $\mathcal{S}_{\mathfrak{g}} \in \mathfrak{T}_{\mathfrak{g}}$, and consider the following definition. 
Definition $2.2\left(\xi, \mathcal{S}_{\mathfrak{g}}\right.$-Pairwise Disjoint). Let $\mathfrak{T}_{\mathfrak{g}}=\left(\Omega, \mathcal{T}_{\mathfrak{g}}\right)$ be a $\mathcal{T}_{\mathfrak{g}}$-space. For some $\sigma \geq 0$ and $\mathcal{S}_{\mathfrak{g}} \supseteq \emptyset$, the families

$$
\begin{aligned}
\mathfrak{g}-\mathrm{F}_{\mathrm{P}}[\sigma] \stackrel{\text { def }}{=}\left\{(\xi, \zeta) \in \mathfrak{T}_{\mathfrak{g}} \times \mathfrak{T}_{\mathfrak{g}}: \mathcal{N}_{\mathfrak{g}}(\xi, \zeta) \geq \sigma\right\}, \\
\mathfrak{g}-\nu-\mathrm{F}_{\mathrm{O}}\left[\mathcal{S}_{\mathfrak{g}}\right] \stackrel{\text { def }}{=}\left\{\left(\mathcal{O}_{\mathfrak{g}, \xi}, \mathcal{O}_{\mathfrak{g}, \zeta}\right) \in \mathcal{T}_{\mathfrak{g}} \times \mathcal{T}_{\mathfrak{g}}: \bigcap_{\lambda=\xi, \zeta} \mathrm{op}_{\mathfrak{g}, \nu}\left(\mathcal{O}_{\mathfrak{g}, \lambda}\right) \subseteq \mathcal{S}_{\mathfrak{g}}\right\}, \\
\left.\mathfrak{g}-\nu-\mathrm{F}_{\mathrm{K}}\left[\mathcal{S}_{\mathfrak{g}}\right] \stackrel{\text { def }}{=}\left\{\left(\mathcal{K}_{\mathfrak{g}, \xi}, \mathcal{K}_{\mathfrak{g}, \zeta}\right) \in \neg \mathcal{T}_{\mathfrak{g}} \times \neg \mathcal{T}_{\mathfrak{g}}: \mathcal{S}_{\mathfrak{g}} \supseteq \bigcap_{\lambda=\xi, \zeta}\right\urcorner \mathrm{op}_{\mathfrak{g}, \nu}\left(\mathcal{K}_{\mathfrak{g}, \lambda}\right)\right\},
\end{aligned}
$$

respectively, denote the collections of pairwise points, and $\mathfrak{g}-\mathcal{T}_{\mathfrak{g}}$-open and $\mathfrak{g}-\mathcal{T}_{\mathfrak{g}}$ closed sets of category $\nu$ in $\mathfrak{T}_{\mathfrak{g}}$. They form the collections of pairwise distinct points, and pairwise disjoint $\mathfrak{g}$ - $\mathcal{T}_{\mathfrak{g}}$-open and $\mathfrak{g}$ - $\mathcal{T}_{\mathfrak{g}}$-closed sets of category $\nu$ whenever $\sigma>0$ and $\mathcal{S}_{\mathfrak{g}}=\emptyset$, respectively.

Granted $\mathfrak{g}-\mathrm{F}_{\mathrm{P}}[\sigma], \mathfrak{g}-\nu-\mathrm{F}_{\mathrm{O}}\left[\mathcal{S}_{\mathfrak{g}}\right]$, and $\mathfrak{g}-\nu-\mathrm{F}_{\mathrm{K}}\left[\mathcal{S}_{\mathfrak{g}}\right]$, the elements of $\left\langle\mathfrak{g}-\nu-\mathrm{T}_{\mathfrak{g}, \alpha}\right\rangle_{\alpha \in \Lambda}$ may well be stated as thus:

Definition $2.3\left(\left\langle\mathfrak{g}-\nu \text { - } \mathrm{T}_{\mathfrak{g}, \alpha}\right\rangle_{\alpha \in \Lambda}\right.$-Axioms $)$. Let $\mathfrak{T}_{\mathfrak{g}}=\left(\Omega, \mathcal{T}_{\mathfrak{g}}\right)$ be a $\mathcal{T}_{\mathfrak{g}}$-space and let $\mathfrak{g}-\mathrm{F}_{\mathrm{P}}[\sigma]$, and $\mathfrak{g}-\nu-\mathrm{F}_{\mathrm{O}}\left[\mathcal{S}_{\mathfrak{g}}\right] \subseteq \mathcal{T}_{\mathfrak{g}} \times \mathcal{T}_{\mathfrak{g}}$ and $\mathfrak{g}-\nu-\mathrm{F}_{\mathrm{K}}\left[\mathcal{S}_{\mathfrak{g}}\right] \subseteq \neg \mathcal{T}_{\mathfrak{g}} \times \neg \mathcal{T}_{\mathfrak{g}}$ be given, where $\sigma \geq 0$ and $\mathcal{S}_{\mathfrak{g}} \supseteq \emptyset$. Then:

- I. $\mathfrak{g}-\nu-\mathrm{T}_{\mathfrak{g}, \mathrm{K}}$-Axiom: For every $(\xi, \zeta) \in \mathfrak{g}-\mathrm{F}_{\mathrm{P}}[\sigma>0]$, there exists a pair $\left(\mathcal{O}_{\mathfrak{g}, \xi}, \mathcal{O}_{\mathfrak{g}, \zeta}\right) \in \mathfrak{g}-\nu-\mathrm{F}_{\mathrm{O}}\left[\mathcal{S}_{\mathfrak{g}} \supset \emptyset\right]$ such that:

$$
\begin{aligned}
{\left[\left(\xi \in \mathrm{op}_{\mathfrak{g}, \nu}\left(\mathcal{O}_{\mathfrak{g}, \xi}\right)\right) \wedge\left(\zeta \notin \mathrm{op}_{\mathfrak{g}, \nu}\left(\mathcal{O}_{\mathfrak{g}, \xi}\right)\right)\right] } & \vee\left[\left(\xi \notin \mathrm{op}_{\mathfrak{g}, \nu}\left(\mathcal{O}_{\mathfrak{g}, \zeta}\right)\right)\right. \\
& \left.\wedge\left(\zeta \in \mathrm{op}_{\mathfrak{g}, \nu}\left(\mathcal{O}_{\mathfrak{g}, \zeta}\right)\right)\right] .
\end{aligned}
$$

- II. $\mathfrak{g}-\nu-\mathrm{T}_{\mathfrak{g}, \mathrm{F}}$-AXIOM: For every $(\xi, \zeta) \in \mathfrak{g}-\mathrm{F}_{\mathrm{P}}[\sigma>0]$, there exists a pair $\left(\mathcal{O}_{\mathfrak{g}, \xi}, \mathcal{O}_{\mathfrak{g}, \zeta}\right) \in \mathfrak{g}-\nu-\mathrm{F}_{\mathrm{O}}\left[\mathcal{S}_{\mathfrak{g}} \supset \emptyset\right]$ such that:

$$
\left[(\xi, \zeta) \in \times_{\lambda=\xi, \zeta} \mathrm{op}_{\mathfrak{g}, \nu}\left(\mathcal{O}_{\mathfrak{g}, \lambda}\right)\right] \wedge\left[(\xi, \zeta) \notin \times_{\lambda=\zeta, \xi} \mathrm{op}_{\mathfrak{g}, \nu}\left(\mathcal{O}_{\mathfrak{g}, \lambda}\right)\right] .
$$

- III. $\mathfrak{g}-\nu-\mathrm{T}_{\mathfrak{g}, \mathrm{H}}$-AXIOM: For every $(\xi, \zeta) \in \mathfrak{g}-\mathrm{F}_{\mathrm{P}}[\sigma>0]$, there exists a pair $\left(\mathcal{O}_{\mathfrak{g}, \xi}, \mathcal{O}_{\mathfrak{g}, \zeta}\right) \in \mathfrak{g}-\nu-\mathrm{F}_{\mathrm{O}}[\emptyset]$ such that:

$$
\left[\xi \in \mathrm{op}_{\mathfrak{g}, \nu}\left(\mathcal{O}_{\mathfrak{g}, \xi}\right)\right] \wedge\left[\zeta \in \mathrm{op}_{\mathfrak{g}, \nu}\left(\mathcal{O}_{\mathfrak{g}, \zeta}\right)\right]
$$

- IV. $\mathfrak{g}-\nu$ - $\mathrm{T}_{\mathfrak{g}, \mathrm{R}}$-Axiom: For every $(\xi, \zeta) \in \mathfrak{g}-\mathrm{F}_{\mathrm{P}}[\sigma>0]$ and $\left(\mathcal{K}_{\mathfrak{g}, \xi}, \mathcal{K}_{\mathfrak{g}, \zeta}\right) \in$ $\mathfrak{g}-\nu-\mathrm{F}_{\mathrm{K}}[\emptyset]$ such that $(\zeta, \xi) \notin\left(\mathcal{K}_{\mathfrak{g}, \xi}, \mathcal{K}_{\mathfrak{g}, \zeta}\right)$, there exists a pair $\left(\mathcal{O}_{\mathfrak{g}, \xi}, \mathcal{O}_{\mathfrak{g}, \zeta}\right) \in$ $\mathfrak{g}-\nu-\mathrm{F}_{\mathrm{O}}[\emptyset]$ such that:

$$
\begin{aligned}
{\left[\left(\neg \mathrm{op}_{\mathfrak{g}, \nu}\left(\mathcal{K}_{\mathfrak{g}, \xi}\right)\right.\right.} & \left.\subset \mathrm{op}_{\mathfrak{g}, \nu}\left(\mathcal{O}_{\mathfrak{g}, \xi}\right)\right) \\
\left.\subset \mathrm{op}_{\mathfrak{g}, \nu}\left(\mathcal{O}_{\mathfrak{g}, \zeta}\right)\right) & \left.\wedge\left(\zeta \in \mathrm{op}_{\mathfrak{g}, \nu}\left(\mathcal{O}_{\mathfrak{g}, \zeta}\right)\right)\right] \vee\left[\left(\neg \mathrm{op}_{\mathfrak{g}, \nu}\left(\mathcal{O}_{\mathfrak{g}, \xi}\right)\right)\right] .
\end{aligned}
$$

- V. $\mathfrak{g}-\nu-\mathrm{T}_{\mathfrak{g}, \mathrm{N}}-$ AxIOM: For every $\left(\mathcal{K}_{\mathfrak{g}, \xi}, \mathcal{K}_{\mathfrak{g}, \zeta}\right) \in \mathfrak{g}-\nu-\mathrm{F}_{\mathrm{K}}[\emptyset]$, there exists a pair $\left(\mathcal{O}_{\mathfrak{g}, \xi}, \mathcal{O}_{\mathfrak{g}, \zeta}\right) \in \mathfrak{g}-\nu-\mathrm{F}_{\mathrm{O}}[\emptyset]$ such that:

$$
\left[\mathrm{op}_{\mathfrak{g}, \nu}\left(\mathcal{O}_{\mathfrak{g}, \xi}\right) \supset \neg \mathrm{op}_{\mathfrak{g}, \nu}\left(\mathcal{K}_{\mathfrak{g}, \xi}\right)\right] \wedge\left[\mathrm{op}_{\mathfrak{g}, \nu}\left(\mathcal{O}_{\mathfrak{g}, \zeta}\right) \supset \neg \mathrm{op}_{\mathfrak{g}, \nu}\left(\mathcal{K}_{\mathfrak{g}, \zeta}\right)\right] .
$$

Granted $\left\langle\mathfrak{g}-\nu-\mathrm{T}_{\mathfrak{g}, \alpha}\right\rangle_{\alpha \in \Lambda}$, we form $\left\langle\mathfrak{g}-\mathrm{T}_{\mathfrak{g}, \alpha}\right\rangle_{\alpha \in \Lambda} \stackrel{\text { def }}{=}\left\langle\bigvee_{\nu \in I_{3}^{0}} \mathfrak{g}-\nu-\mathrm{T}_{\mathfrak{g}, \alpha}\right\rangle_{\alpha \in \Lambda}$, and define the $\mathfrak{g}-T_{\mathfrak{g}, K}, \mathfrak{g}-T_{\mathfrak{g}, \mathrm{F}}, \mathfrak{g}-\mathrm{T}_{\mathfrak{g}, \mathrm{H}}, \mathfrak{g}-\mathrm{T}_{\mathfrak{g}, \mathrm{R}}$, and $\mathfrak{g}-\mathrm{T}_{\mathfrak{g}, N}$-axioms as thus. 


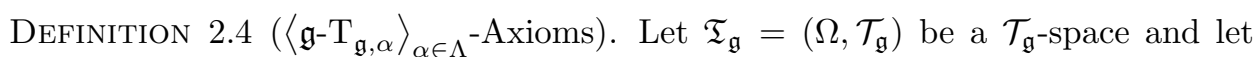
$\mathfrak{g}-\mathrm{F}_{\mathrm{P}}[\sigma], \mathfrak{g}-\mathrm{F}_{\mathrm{O}}\left[\mathcal{S}_{\mathfrak{g}}\right]=\bigcup_{\nu \in I_{3}^{0}} \mathfrak{g}-\nu-\mathrm{F}_{\mathrm{O}}\left[\mathcal{S}_{\mathfrak{g}}\right] \subseteq \mathcal{T}_{\mathfrak{g}} \times \mathcal{T}_{\mathfrak{g}}, \mathfrak{g}-\mathrm{F}_{\mathrm{K}}\left[\mathcal{S}_{\mathfrak{g}}\right]=\bigcup_{\nu \in I_{3}^{0}} \mathfrak{g}-\nu-\mathrm{F}_{\mathrm{K}}\left[\mathcal{S}_{\mathfrak{g}}\right] \subseteq$ $\neg \mathcal{T}_{\mathfrak{g}} \times \neg \mathcal{T}_{\mathfrak{g}}$ be given, where $\sigma \geq 0$ and $\mathcal{S}_{\mathfrak{g}} \supseteq \emptyset$. Then:

- I. $\mathfrak{g}-\mathrm{T}_{\mathfrak{g}, \mathrm{K}}$-Axiom: For every $(\xi, \zeta) \in \mathfrak{g}-\mathrm{F}_{\mathrm{P}}[\sigma>0]$, there exists $\left(\mathcal{O}_{\mathfrak{g}, \xi}, \mathcal{O}_{\mathfrak{g}, \zeta}\right) \in$ $\mathfrak{g}-\mathrm{F}_{\mathrm{O}}\left[\mathcal{S}_{\mathfrak{g}} \supset \emptyset\right]$ such that:

$$
\begin{aligned}
{\left[\left(\xi \in \mathrm{op}_{\mathfrak{g}}\left(\mathcal{O}_{\mathfrak{g}, \xi}\right)\right) \wedge\left(\zeta \notin \mathrm{op}_{\mathfrak{g}}\left(\mathcal{O}_{\mathfrak{g}, \xi}\right)\right)\right] } & \vee\left[\left(\xi \notin \mathrm{op}_{\mathfrak{g}}\left(\mathcal{O}_{\mathfrak{g}, \zeta}\right)\right)\right. \\
& \left.\wedge\left(\zeta \in \mathrm{op}_{\mathfrak{g}}\left(\mathcal{O}_{\mathfrak{g}, \zeta}\right)\right)\right] .
\end{aligned}
$$

- II. $\mathfrak{g}-\mathrm{T}_{\mathfrak{g}, \mathrm{F}}$-AXIOM: For every $(\xi, \zeta) \in \mathfrak{g}-\mathrm{F}_{\mathrm{P}}[\sigma>0]$, there exists a pair $\left(\mathcal{O}_{\mathfrak{g}, \xi}, \mathcal{O}_{\mathfrak{g}, \zeta}\right) \in \mathfrak{g}-\mathrm{F}_{\mathrm{O}}\left[\mathcal{S}_{\mathfrak{g}} \supset \emptyset\right]$ such that:

$$
\left[(\xi, \zeta) \in \times_{\lambda=\xi, \zeta} \mathrm{op}_{\mathfrak{g}}\left(\mathcal{O}_{\mathfrak{g}, \lambda}\right)\right] \wedge\left[(\xi, \zeta) \notin \times_{\lambda=\zeta, \xi} \mathrm{op}_{\mathfrak{g}}\left(\mathcal{O}_{\mathfrak{g}, \lambda}\right)\right] .
$$

- III. $\mathfrak{g}-\mathrm{T}_{\mathfrak{g}, \mathrm{H}^{-}}$-Axiom: For every $(\xi, \zeta) \in \mathfrak{g}-\mathrm{F}_{\mathrm{P}}[\sigma>0]$, there exists a pair $\left(\mathcal{O}_{\mathfrak{g}, \xi}, \mathcal{O}_{\mathfrak{g}, \zeta}\right) \in \mathfrak{g}-\mathrm{F}_{\mathrm{O}}[\emptyset]$ such that:

$$
\left[\xi \in \mathrm{op}_{\mathfrak{g}}\left(\mathcal{O}_{\mathfrak{g}, \xi}\right)\right] \wedge\left[\zeta \in \mathrm{op}_{\mathfrak{g}}\left(\mathcal{O}_{\mathfrak{g}, \zeta}\right)\right] .
$$

- IV. $\mathfrak{g}$-T $\mathrm{T}_{\mathfrak{g}, \mathrm{R}}$-Axiom: For every $(\xi, \zeta) \in \mathfrak{g}-\mathrm{F}_{\mathrm{P}}[\sigma>0]$ and $\left(\mathcal{K}_{\mathfrak{g}, \xi}, \mathcal{K}_{\mathfrak{g}, \zeta}\right) \in$ $\mathfrak{g}-\mathrm{F}_{\mathrm{K}}[\emptyset]$ such that $(\zeta, \xi) \notin\left(\mathcal{K}_{\mathfrak{g}, \xi}, \mathcal{K}_{\mathfrak{g}, \zeta}\right)$, there exists $\left(\mathcal{O}_{\mathfrak{g}, \xi}, \mathcal{O}_{\mathfrak{g}, \zeta}\right) \in \mathfrak{g}-\mathrm{F}_{\mathrm{O}}[\emptyset]$ such that:

$$
\begin{aligned}
{\left[\left(\neg \mathrm{op}_{\mathfrak{g}}\left(\mathcal{K}_{\mathfrak{g}, \xi}\right) \subset \mathrm{op}_{\mathfrak{g}}\left(\mathcal{O}_{\mathfrak{g}, \xi}\right)\right)\right.} & \left.\wedge\left(\zeta \in \mathrm{op}_{\mathfrak{g}}\left(\mathcal{O}_{\mathfrak{g}, \zeta}\right)\right)\right] \vee\left[\left(\neg \mathrm{op}_{\mathfrak{g}}\left(\mathcal{K}_{\mathfrak{g}, \zeta}\right)\right.\right. \\
& \left.\left.\subset \mathrm{op}_{\mathfrak{g}}\left(\mathcal{O}_{\mathfrak{g}, \zeta}\right)\right) \wedge\left(\xi \in \mathrm{op}_{\mathfrak{g}}\left(\mathcal{O}_{\mathfrak{g}, \xi}\right)\right)\right] .
\end{aligned}
$$

- V. $\mathfrak{g}-\mathrm{T}_{\mathfrak{g}, \mathrm{N}}$-AXIOM: For every $\left(\mathcal{K}_{\mathfrak{g}, \xi}, \mathcal{K}_{\mathfrak{g}, \zeta}\right) \in \mathfrak{g}-\mathrm{F}_{\mathrm{K}}[\emptyset]$, there exists a pair $\left(\mathcal{O}_{\mathfrak{g}, \xi}, \mathcal{O}_{\mathfrak{g}, \zeta}\right) \in \mathfrak{g}-\mathrm{F}_{\mathrm{O}}[\emptyset]$ such that:

$$
\left[\operatorname{op}_{\mathfrak{g}}\left(\mathcal{O}_{\mathfrak{g}, \xi}\right) \supset \neg \mathrm{op}_{\mathfrak{g}}\left(\mathcal{K}_{\mathfrak{g}, \xi}\right)\right] \wedge\left[\operatorname{op}_{\mathfrak{g}}\left(\mathcal{O}_{\mathfrak{g}, \zeta}\right) \supset \neg \mathrm{op}_{\mathfrak{g}}\left(\mathcal{K}_{\mathfrak{g}, \zeta}\right)\right] .
$$

In the following sections, the main results of the theory of $\mathfrak{g}$ - $\mathfrak{T}_{\mathfrak{g}}$-maps are presented.

\section{Main Results}

A necessary and sufficient condition for a $\mathcal{T}_{\mathfrak{g}}$-space $\mathfrak{T}_{\mathfrak{g}}=\left(\Omega, \mathcal{T}_{\mathfrak{g}}\right)$ to be a $\mathfrak{g}-\mathcal{T}_{\mathfrak{g}}^{(\mathrm{K})}$ space $\mathfrak{g}-\mathfrak{T}_{\mathfrak{g}}^{(\mathrm{K})}=\left(\Omega, \mathfrak{g}-\mathcal{T}_{\mathfrak{g}}^{(\mathrm{K})}\right)$ may be given in terms of the complementary $\mathfrak{g}$-operator $\neg \mathrm{op}_{\mathfrak{g}}: \mathcal{P}(\Omega) \rightarrow \mathcal{P}(\Omega)$ and any pairs $(\{\xi\},\{\zeta\}) \subset \mathfrak{T}_{\mathfrak{g}} \times \mathfrak{T}_{\mathfrak{g}}$ of unit sets.

THEOREM 3.1. A $\mathcal{T}_{\mathfrak{g}}$-space $\mathfrak{T}_{\mathfrak{g}}=\left(\Omega, \mathcal{T}_{\mathfrak{g}}\right)$ is said to be a $\mathfrak{g}-\mathcal{T}_{\mathfrak{g}}^{(\mathrm{K})}$-space $\mathfrak{g}-\mathfrak{T}_{\mathfrak{g}}^{(\mathrm{K})}=$ $\left(\Omega, \mathfrak{g}-\mathcal{T}_{\mathfrak{g}}^{(\mathrm{K})}\right)$ if and only if the following condition holds:

$$
\neg \mathrm{op}_{\mathfrak{g}}(\{\xi\}) \neq \neg \mathrm{op}_{\mathfrak{g}}(\{\zeta\}) \quad \forall(\xi, \zeta) \in \mathfrak{g}-\mathrm{F}_{\mathrm{P}}[\sigma>0] .
$$

Proof. Necessity. Let the $\mathcal{T}_{\mathfrak{g}}$-space $\mathfrak{T}_{\mathfrak{g}}$ be a $\mathfrak{g}-\mathcal{T}_{\mathfrak{g}}^{(\mathrm{K})}$-space $\mathfrak{g}$ - $\mathfrak{T}_{\mathfrak{g}}^{(\mathrm{K})}$. Then, for every $(\xi, \zeta) \in \mathfrak{g}-\mathrm{F}_{\mathrm{P}}[\sigma>0]$, there exists $\left(\mathcal{O}_{\mathfrak{g}, \xi}, \mathcal{O}_{\mathfrak{g}, \zeta}\right) \in \mathfrak{g}-\mathrm{F}_{\mathrm{O}}\left[\mathcal{S}_{\mathfrak{g}} \supset \emptyset\right]$ such that:

Consequently,

$$
\begin{aligned}
{\left[\left(\xi \in \mathrm{op}_{\mathfrak{g}}\left(\mathcal{O}_{\mathfrak{g}, \xi}\right)\right) \wedge\left(\zeta \notin \mathrm{op}_{\mathfrak{g}}\left(\mathcal{O}_{\mathfrak{g}, \xi}\right)\right)\right] } & \vee\left[\left(\xi \notin \mathrm{op}_{\mathfrak{g}}\left(\mathcal{O}_{\mathfrak{g}, \zeta}\right)\right)\right. \\
& \left.\wedge\left(\zeta \in \mathrm{op}_{\mathfrak{g}}\left(\mathcal{O}_{\mathfrak{g}, \zeta}\right)\right)\right] .
\end{aligned}
$$

$$
\begin{aligned}
{\left[\left(\xi \notin \complement\left(\mathrm{op}_{\mathfrak{g}}\left(\mathcal{O}_{\mathfrak{g}, \xi}\right)\right)\right) \wedge\left(\zeta \in \complement\left(\mathrm{op}_{\mathfrak{g}}\left(\mathcal{O}_{\mathfrak{g}, \xi}\right)\right)\right)\right] } & \vee\left[\left(\xi \in \complement\left(\mathrm{op}_{\mathfrak{g}}\left(\mathcal{O}_{\mathfrak{g}, \zeta}\right)\right)\right)\right. \\
& \left.\wedge\left(\zeta \notin \complement\left(\operatorname{op}_{\mathfrak{g}}\left(\mathcal{O}_{\mathfrak{g}, \zeta}\right)\right)\right)\right]
\end{aligned}
$$


implying $\complement\left(\operatorname{op}_{\mathfrak{g}}\left(\mathcal{O}_{\mathfrak{g}, \xi}\right)\right), \complement\left(\operatorname{op}_{\mathfrak{g}}\left(\mathcal{O}_{\mathfrak{g}, \zeta}\right)\right) \in \mathfrak{g}-\mathrm{K}\left[\mathfrak{T}_{\mathfrak{g}}\right]$, respectively, are $\mathfrak{g}-\mathfrak{T}_{\mathfrak{g}}$-closed sets containing $\zeta \in \mathfrak{T}_{\mathfrak{g}}$ and $\xi \in \mathfrak{T}_{\mathfrak{g}}$. Thus, there exists $\left(\mathcal{K}_{\mathfrak{g}, \xi}, \mathcal{K}_{\mathfrak{g}, \zeta}\right) \in \neg \mathcal{T}_{\mathfrak{g}} \times \neg \mathcal{T}_{\mathfrak{g}}$ such that $\complement\left(\operatorname{op}_{\mathfrak{g}}\left(\mathcal{O}_{\mathfrak{g}, \xi}\right)\right) \supseteq \neg \mathrm{op}_{\mathfrak{g}}\left(\mathcal{K}_{\mathfrak{g}, \zeta}\right)$ and $\complement\left(\mathrm{op}_{\mathfrak{g}}\left(\mathcal{O}_{\mathfrak{g}, \zeta}\right)\right) \supseteq \neg \mathrm{op}_{\mathfrak{g}}\left(\mathcal{K}_{\mathfrak{g}, \xi}\right)$. But, for every $\lambda \in\{\xi, \zeta\}, \complement(\{\lambda\}) \supseteq \complement\left(\mathrm{op}_{\mathfrak{g}}(\{\lambda\})\right) \supseteq \complement\left(\mathrm{op}_{\mathfrak{g}}\left(\mathcal{O}_{\mathfrak{g}, \lambda}\right)\right)$ and $\neg \mathrm{op}_{\mathfrak{g}}\left(\mathcal{K}_{\mathfrak{g}, \lambda}\right) \supseteq \neg \mathrm{op}_{\mathfrak{g}}(\{\lambda\})$. Therefore, $\complement(\{\xi\}) \supseteq \neg \mathrm{op}_{\mathfrak{g}}(\{\zeta\})$ and $\complement(\{\zeta\}) \supseteq \neg \mathrm{op}_{\mathfrak{g}}(\{\xi\})$. Since, $\complement(\{\xi\}) \neq \grave{\complement}(\{\zeta\})$, it follows that $\neg \mathrm{op}_{\mathfrak{g}}(\{\xi\}) \neq \neg \mathrm{op}_{\mathfrak{g}}(\{\zeta\})$.

Sufficiency. Conversely, suppose the condition $\neg \mathrm{op}_{\mathfrak{g}}(\{\xi\}) \neq \neg \mathrm{op}_{\mathfrak{g}}(\{\zeta\})$ holds for every $(\xi, \zeta) \in \mathfrak{g}-\mathrm{F}_{\mathrm{P}}[\sigma>0]$. Then there exists $\eta \in \mathfrak{T}_{\mathfrak{g}}$ such that

$$
\begin{aligned}
{\left[\left(\eta \in \neg \mathrm{op}_{\mathfrak{g}}(\{\xi\})\right) \wedge\left(\eta \notin \neg \mathrm{op}_{\mathfrak{g}}(\{\zeta\})\right)\right] } & \vee\left[\left(\eta \notin \neg \mathrm{op}_{\mathfrak{g}}(\{\xi\})\right)\right. \\
& \left.\wedge\left(\eta \in \neg \mathrm{op}_{\mathfrak{g}}(\{\zeta\})\right)\right] .
\end{aligned}
$$

If $\left[\xi \in \neg \mathrm{op}_{\mathfrak{g}}(\{\zeta\})\right] \wedge\left[\zeta \in \neg \mathrm{op}_{\mathfrak{g}}(\{\xi\})\right]$, then

$$
\left[\neg \mathrm{op}_{\mathfrak{g}}(\{\xi\}) \subseteq \neg \mathrm{op}_{\mathfrak{g}}(\{\zeta\})\right] \wedge\left[\neg \mathrm{op}_{\mathfrak{g}}(\{\zeta\}) \subseteq \neg \mathrm{op}_{\mathfrak{g}}(\{\xi\})\right] .
$$

Consequently,

$$
\begin{aligned}
{\left[\left(\eta \in \neg \mathrm{op}_{\mathfrak{g}}(\{\zeta\})\right) \wedge\left(\eta \notin \neg \mathrm{op}_{\mathfrak{g}}(\{\zeta\})\right)\right] } & \vee\left[\left(\eta \notin \neg \mathrm{op}_{\mathfrak{g}}(\{\xi\})\right)\right. \\
& \left.\wedge\left(\eta \in \neg \mathrm{op}_{\mathfrak{g}}(\{\xi\})\right)\right] .
\end{aligned}
$$

This is a contradiction; hence, $\left[\xi \notin \neg \mathrm{op}_{\mathfrak{g}}(\{\zeta\})\right] \wedge\left[\zeta \notin \neg \mathrm{op}_{\mathfrak{g}}(\{\xi\})\right]$, implying

$$
\left[\xi \in \complement\left(\neg \mathrm{op}_{\mathfrak{g}}(\{\zeta\})\right)\right] \wedge\left[\zeta \in \complement\left(\neg \mathrm{op}_{\mathfrak{g}}(\{\xi\})\right)\right] .
$$

Since $\complement\left(\neg \mathrm{op}_{\mathfrak{g}}(\{\zeta\})\right), \complement\left(\neg \mathrm{op}_{\mathfrak{g}}(\{\xi\})\right) \in \mathfrak{g}$-O $\left[\mathfrak{T}_{\mathfrak{g}}\right]$, respectively, are $\mathfrak{g}$ - $\mathfrak{T}_{\mathfrak{g}}$-open sets containing $\xi \in \mathfrak{T}_{\mathfrak{g}}$ and $\zeta \in \mathfrak{T}_{\mathfrak{g}}$, there exists $\left(\mathcal{O}_{\mathfrak{g}, \xi}, \mathcal{O}_{\mathfrak{g}, \zeta}\right) \in \mathcal{T}_{\mathfrak{g}} \times \mathcal{T}_{\mathfrak{g}}$ such that $\complement\left(\neg \mathrm{op}_{\mathfrak{g}}(\{\zeta\})\right) \subseteq \mathrm{op}_{\mathfrak{g}}\left(\mathcal{O}_{\mathfrak{g}, \xi}\right)$ and $\complement\left(\neg \mathrm{op}_{\mathfrak{g}}(\{\xi\})\right) \subseteq \mathrm{op}_{\mathfrak{g}}\left(\mathcal{O}_{\mathfrak{g}, \zeta}\right)$. Hence, for every $(\xi, \zeta) \in \mathfrak{g}-\mathrm{F}_{\mathrm{P}}[\sigma>0]$, there exists $\left(\mathcal{O}_{\mathfrak{g}, \xi}, \mathcal{O}_{\mathfrak{g}, \zeta}\right) \in \mathfrak{g}-\mathrm{F}_{\mathrm{O}}\left[\mathcal{S}_{\mathfrak{g}} \supset \emptyset\right]$ such that:

$$
\begin{aligned}
{\left[\left(\xi \in \mathrm{op}_{\mathfrak{g}}\left(\mathcal{O}_{\mathfrak{g}, \xi}\right)\right) \wedge\left(\zeta \notin \mathrm{op}_{\mathfrak{g}}\left(\mathcal{O}_{\mathfrak{g}, \xi}\right)\right)\right] } & \vee\left[\left(\xi \notin \mathrm{op}_{\mathfrak{g}}\left(\mathcal{O}_{\mathfrak{g}, \zeta}\right)\right)\right. \\
& \left.\wedge\left(\zeta \in \mathrm{op}_{\mathfrak{g}}\left(\mathcal{O}_{\mathfrak{g}, \zeta}\right)\right)\right] .
\end{aligned}
$$

Therefore, $\mathfrak{T}_{\mathfrak{g}}=\left(\Omega, \mathcal{T}_{\mathfrak{g}}\right)$ is a $\mathfrak{g}-\mathcal{T}_{\mathfrak{g}}^{(\mathrm{K})}$-space $\mathfrak{g}-\mathfrak{T}_{\mathfrak{g}}^{(\mathrm{K})}=\left(\Omega, \mathfrak{g}-\mathcal{T}_{\mathfrak{g}}^{(\mathrm{K})}\right)$; this completes the proof of the theorem.

Q.E.D.

A necessary and sufficient condition for a $\mathcal{T}_{\mathfrak{g}}$-space $\mathfrak{T}_{\mathfrak{g}}=\left(\Omega, \mathcal{T}_{\mathfrak{g}}\right)$ to be a $\mathfrak{g}-\mathcal{T}_{\mathfrak{g}}^{(\mathrm{F})}$ space $\mathfrak{g}-\mathfrak{T}_{\mathfrak{g}}^{(\mathrm{F})}=\left(\Omega, \mathfrak{g}-\mathcal{T}_{\mathfrak{g}}^{(\mathrm{F})}\right)$ may be given in terms of the complementary $\mathfrak{g}$-operator $\neg \mathrm{op}_{\mathfrak{g}}: \mathcal{P}(\Omega) \rightarrow \mathcal{P}(\Omega)$ and a unit set $\{\xi\} \subset \mathfrak{T}_{\mathfrak{g}}$.

THEOREM 3.2. A $\mathcal{T}_{\mathfrak{g}}$-space $\mathfrak{T}_{\mathfrak{g}}=\left(\Omega, \mathcal{T}_{\mathfrak{g}}\right)$ is said to be a $\mathfrak{g}-\mathcal{T}_{\mathfrak{g}}^{(\mathrm{F})}$-space $\mathfrak{g}$ - $\mathfrak{T}_{\mathfrak{g}}^{(\mathrm{F})}=$ $\left(\Omega, \mathfrak{g}-\mathcal{T}_{\mathfrak{g}}^{(\mathrm{F})}\right)$ if and only if the following condition holds:

$$
\{\xi\} \supseteq \neg \mathrm{op}_{\mathfrak{g}}(\{\xi\}) \quad \forall \xi \in \mathfrak{T}_{\mathfrak{g}} .
$$

PROOF. Necessity. Let the $\mathcal{T}_{\mathfrak{g}}$-space $\mathfrak{T}_{\mathfrak{g}}$ be a $\mathfrak{g}-\mathcal{T}_{\mathfrak{g}}^{(\mathrm{F})}$-space $\mathfrak{g}$ - $\mathfrak{T}_{\mathfrak{g}}^{(\mathrm{F})}$. Then, for every $(\xi, \zeta) \in \mathfrak{g}-\mathrm{F}_{\mathrm{P}}[\sigma>0]$, there exists $\left(\mathcal{O}_{\mathfrak{g}, \xi}, \mathcal{O}_{\mathfrak{g}, \zeta}\right) \in \mathfrak{g}-\mathrm{F}_{\mathrm{O}}\left[\mathcal{S}_{\mathfrak{g}} \supset \emptyset\right]$ such that:

$$
\left[(\xi, \zeta) \in \times_{\lambda=\xi, \zeta} \mathrm{op}_{\mathfrak{g}}\left(\mathcal{O}_{\mathfrak{g}, \lambda}\right)\right] \wedge\left[(\xi, \zeta) \notin \times_{\lambda=\zeta, \xi} \mathrm{op}_{\mathfrak{g}}\left(\mathcal{O}_{\mathfrak{g}, \lambda}\right)\right] .
$$

Consequently,

$$
\left[(\xi, \zeta) \notin \times_{\lambda=\xi, \zeta} \complement\left(\mathrm{op}_{\mathfrak{g}}\left(\mathcal{O}_{\mathfrak{g}, \lambda}\right)\right)\right] \wedge\left[(\xi, \zeta) \in \times_{\lambda=\zeta, \xi} \complement\left(\mathrm{op}_{\mathfrak{g}}\left(\mathcal{O}_{\mathfrak{g}, \lambda}\right)\right)\right] .
$$


Since, for every $\lambda \in\{\xi, \zeta\}, \complement\left(\operatorname{op}_{\mathfrak{g}}\left(\mathcal{O}_{\mathfrak{g}, \lambda}\right)\right) \in \mathfrak{g}-\mathrm{K}\left[\mathfrak{T}_{\mathfrak{g}}\right]$ is a $\mathfrak{g}$ - $\mathfrak{T}_{\mathfrak{g}}$-closed set, there exists $\left(\mathcal{K}_{\mathfrak{g}, \xi}, \mathcal{K}_{\mathfrak{g}, \zeta}\right) \in \neg \mathcal{T}_{\mathfrak{g}} \times \neg \mathcal{T}_{\mathfrak{g}}$ such that $\complement\left(\operatorname{op}_{\mathfrak{g}}\left(\mathcal{O}_{\mathfrak{g}, \xi}\right)\right) \supseteq \neg \mathrm{op}_{\mathfrak{g}}\left(\mathcal{K}_{\mathfrak{g}, \zeta}\right)$ and $\complement\left(\operatorname{op}_{\mathfrak{g}}\left(\mathcal{O}_{\mathfrak{g}, \zeta}\right)\right) \supseteq$ $\neg \mathrm{op}_{\mathfrak{g}}\left(\mathcal{K}_{\mathfrak{g}, \xi}\right)$. But, for every $\lambda \in\{\xi, \zeta\}, \neg \mathrm{op}_{\mathfrak{g}}\left(\mathcal{K}_{\mathfrak{g}, \lambda}\right) \supseteq \neg \mathrm{op}_{\mathfrak{g}}(\{\lambda\})$. Therefore

$$
\left.\left[(\xi, \zeta) \in \times_{\lambda=\xi, \zeta} \neg \mathrm{op}_{\mathfrak{g}}(\{\lambda\})\right] \wedge\left[(\xi, \zeta) \notin \times_{\lambda=\zeta, \xi}\right\urcorner \mathrm{op}_{\mathfrak{g}}(\{\lambda\})\right] .
$$

Hence, for every $\xi \in \mathfrak{T}_{\mathfrak{g}},\{\xi\} \supseteq \neg \mathrm{op}_{\mathfrak{g}}(\{\xi\})$.

Sufficiency. Conversely, suppose the condition $\{\xi\} \supseteq \neg \mathrm{op}_{\mathfrak{g}}(\{\xi\})$ holds for every $\xi \in \mathfrak{T}_{\mathfrak{g}}$. Let $(\xi, \zeta) \in \mathfrak{T}_{\mathfrak{g}} \times \mathfrak{T}_{\mathfrak{g}}$ such that $\xi \neq \zeta$. Then

$$
\left[(\xi, \zeta) \notin \times_{\lambda=\xi, \zeta} \complement(\{\lambda\})\right] \wedge\left[(\xi, \zeta) \in \times_{\lambda=\zeta, \xi} \complement(\{\lambda\})\right] .
$$

But, for every $\lambda \in\{\xi, \zeta\}, \complement(\{\lambda\}) \subseteq \complement\left(\neg \mathrm{op}_{\mathfrak{g}}(\{\lambda\})\right)$, and $\complement\left(\neg \mathrm{op}_{\mathfrak{g}}(\{\lambda\})\right) \in \mathfrak{g}-\mathrm{O}\left[\mathfrak{T}_{\mathfrak{g}}\right]$ is a $\mathfrak{g}-\mathfrak{T}_{\mathfrak{g}}$-open set. Thus, there exists $\left(\mathcal{O}_{\mathfrak{g}, \xi}, \mathcal{O}_{\mathfrak{g}, \zeta}\right) \in \mathcal{T}_{\mathfrak{g}} \times \mathcal{T}_{\mathfrak{g}}$ such that $\complement\left(\neg \mathrm{op}_{\mathfrak{g}}(\{\xi\})\right) \subseteq$ $\mathrm{op}_{\mathfrak{g}}\left(\mathcal{O}_{\mathfrak{g}, \zeta}\right)$ and $\complement\left(\neg \mathrm{op}_{\mathfrak{g}}(\{\zeta\})\right) \subseteq \mathrm{op}_{\mathfrak{g}}\left(\mathcal{O}_{\mathfrak{g}, \xi}\right)$. By substitution, it thus follows that, for every $(\xi, \zeta) \in \mathfrak{g}-\mathrm{F}_{\mathrm{P}}[\sigma>0]$, there exists $\left(\mathcal{O}_{\mathfrak{g}, \xi}, \mathcal{O}_{\mathfrak{g}, \zeta}\right) \in \mathfrak{g}-\mathrm{F}_{\mathrm{O}}\left[\mathcal{S}_{\mathfrak{g}} \supset \emptyset\right]$ such that:

$$
\left[(\xi, \zeta) \in \times_{\lambda=\xi, \zeta} \mathrm{op}_{\mathfrak{g}}\left(\mathcal{O}_{\mathfrak{g}, \lambda}\right)\right] \wedge\left[(\xi, \zeta) \notin \times_{\lambda=\zeta, \xi} \mathrm{op}_{\mathfrak{g}}\left(\mathcal{O}_{\mathfrak{g}, \lambda}\right)\right] .
$$

Therefore, $\mathfrak{T}_{\mathfrak{g}}=\left(\Omega, \mathcal{T}_{\mathfrak{g}}\right)$ is a $\mathfrak{g}-\mathcal{T}_{\mathfrak{g}}^{(\mathrm{F})}$-space $\mathfrak{g}-\mathfrak{T}_{\mathfrak{g}}^{(\mathrm{F})}=\left(\Omega, \mathfrak{g}-\mathcal{T}_{\mathfrak{g}}^{(\mathrm{F})}\right)$; this completes the proof of the theorem.

Q.E.D.

PROPOSITION 3.3. If $\mathfrak{T}_{\mathfrak{g}}=\left(\Omega, \mathcal{T}_{\mathfrak{g}}\right)$ is a $\mathfrak{g}-\mathcal{T}_{\mathfrak{g}}^{(\mathrm{F})}$-space $\mathfrak{g}-\mathfrak{T}_{\mathfrak{g}}^{(\mathrm{F})}=\left(\Omega, \mathfrak{g}-\mathcal{T}_{\mathfrak{g}}^{(\mathrm{F})}\right)$, then it is a $\mathfrak{g}-\mathcal{T}_{\mathfrak{g}}^{(\mathrm{K})}$-space $\mathfrak{g}-\mathfrak{T}_{\mathfrak{g}}^{(\mathrm{K})}=\left(\Omega, \mathfrak{g}-\mathcal{T}_{\mathfrak{g}}^{(\mathrm{K})}\right)$.

Proof. Let $\mathfrak{T}_{\mathfrak{g}}=\left(\Omega, \mathcal{T}_{\mathfrak{g}}\right)$ be a $\mathfrak{g}-\mathcal{T}_{\mathfrak{g}}^{(\mathrm{F})}$-space $\mathfrak{g}-\mathfrak{T}_{\mathfrak{g}}^{(\mathrm{F})}=\left(\Omega, \mathfrak{g}-\mathcal{T}_{\mathfrak{g}}^{(\mathrm{F})}\right)$. Then, for every $(\xi, \zeta) \in \mathfrak{g}-\mathrm{F}_{\mathrm{P}}[\sigma>0]$, there exists a pair $\left(\mathcal{O}_{\mathfrak{g}, \xi}, \mathcal{O}_{\mathfrak{g}, \zeta}\right) \in \mathfrak{g}-\mathrm{F}_{\mathrm{O}}\left[\mathcal{S}_{\mathfrak{g}} \supset \emptyset\right]$ such that:

$$
\left[(\xi, \zeta) \in \times_{\lambda=\xi, \zeta} \mathrm{op}_{\mathfrak{g}}\left(\mathcal{O}_{\mathfrak{g}, \lambda}\right)\right] \wedge\left[(\xi, \zeta) \notin \times_{\lambda=\zeta, \xi} \mathrm{op}_{\mathfrak{g}}\left(\mathcal{O}_{\mathfrak{g}, \lambda}\right)\right]
$$

Set $\mathrm{P}(\xi, \zeta)=\left(\xi \in \mathrm{op}_{\mathfrak{g}}\left(\mathcal{O}_{\mathfrak{g}, \xi}\right)\right) \wedge\left(\zeta \notin \mathrm{op}_{\mathfrak{g}}\left(\mathcal{O}_{\mathfrak{g}, \xi}\right)\right)$. Then, the above logical statement is equivalent to $\mathrm{P}(\xi, \zeta) \wedge \mathrm{P}(\zeta, \xi)$. But, logically,

$$
\mathrm{P}(\xi, \zeta) \vee \mathrm{P}(\zeta, \xi)=\mathrm{P}(\xi, \zeta) \vee \mathrm{P}(\zeta, \xi) \vee(\mathrm{P}(\xi, \zeta) \wedge \mathrm{P}(\zeta, \xi))
$$

Consequently, $\mathrm{P}(\xi, \zeta) \vee \mathrm{P}(\zeta, \xi) \leftarrow \mathrm{P}(\xi, \zeta) \wedge \mathrm{P}(\zeta, \xi)$, from which it then follows that, if $\mathfrak{T}_{\mathfrak{g}}=\left(\Omega, \mathcal{T}_{\mathfrak{g}}\right)$ is a $\mathfrak{g}-\mathcal{T}_{\mathfrak{g}}^{(\mathrm{F})}$-space $\mathfrak{g}-\mathfrak{T}_{\mathfrak{g}}^{(\mathrm{F})}=\left(\Omega, \mathfrak{g}-\mathcal{T}_{\mathfrak{g}}^{(\mathrm{F})}\right)$, then for every $(\xi, \zeta) \in$ $\mathfrak{g}-\mathrm{F}_{\mathrm{P}}[\sigma>0]$, there exists $\left(\mathcal{O}_{\mathfrak{g}, \xi}, \mathcal{O}_{\mathfrak{g}, \zeta}\right) \in \mathfrak{g}-\mathrm{F}_{\mathrm{O}}\left[\mathcal{S}_{\mathfrak{g}} \supset \emptyset\right]$ such that:

$$
\begin{aligned}
{\left[\left(\xi \in \mathrm{op}_{\mathfrak{g}}\left(\mathcal{O}_{\mathfrak{g}, \xi}\right)\right) \wedge\left(\zeta \notin \mathrm{op}_{\mathfrak{g}}\left(\mathcal{O}_{\mathfrak{g}, \xi}\right)\right)\right] } & \vee\left[\left(\xi \notin \mathrm{op}_{\mathfrak{g}}\left(\mathcal{O}_{\mathfrak{g}, \zeta}\right)\right)\right. \\
& \left.\wedge\left(\zeta \in \mathrm{op}_{\mathfrak{g}}\left(\mathcal{O}_{\mathfrak{g}, \zeta}\right)\right)\right],
\end{aligned}
$$

the logical statement characterising $\mathfrak{T}_{\mathfrak{g}}=\left(\Omega, \mathcal{T}_{\mathfrak{g}}\right)$ as a $\mathfrak{g}-\mathcal{T}_{\mathfrak{g}}^{(\mathrm{K})}$-space $\mathfrak{g}-\mathfrak{T}_{\mathfrak{g}}^{(\mathrm{K})}=$ $\left(\Omega, \mathfrak{g}-\mathcal{T}_{\mathfrak{g}}^{(\mathrm{K})}\right)$.

Q.E.D.

A necessary and sufficient condition for a $\mathcal{T}_{\mathfrak{g}}$-space $\mathfrak{T}_{\mathfrak{g}}=\left(\Omega, \mathcal{T}_{\mathfrak{g}}\right)$ to be a $\mathfrak{g}-\mathcal{T}_{\mathfrak{g}}^{(\mathrm{H})}$ space $\mathfrak{g}-\mathfrak{T}_{\mathfrak{g}}^{(\mathrm{H})}=\left(\Omega, \mathfrak{g}-\mathcal{T}_{\mathfrak{g}}^{(\mathrm{H})}\right)$ may be given in terms of the $\mathfrak{g}$-operator op $\mathfrak{g}: \mathcal{P}(\Omega) \rightarrow$ $\mathcal{P}(\Omega)$, a unit set $\{\xi\} \subset \mathfrak{T}_{\mathfrak{g}}$, and $\mathcal{T}_{\mathfrak{g}}$-closed sets.

TheOREM 3.4. A $\mathcal{T}_{\mathfrak{g}}$-space $\mathfrak{T}_{\mathfrak{g}}=\left(\Omega, \mathcal{T}_{\mathfrak{g}}\right)$ is said to be a $\mathfrak{g}-\mathcal{T}_{\mathfrak{g}}^{(\mathrm{H})}$-space $\mathfrak{g}$ - $\mathfrak{T}_{\mathfrak{g}}^{(\mathrm{H})}=$ $\left(\Omega, \mathfrak{g}-\mathcal{T}_{\mathfrak{g}}^{(\mathrm{H})}\right)$ if and only if the following conditions hold:

$$
\{\xi\}=\bigcap_{\mathcal{K}_{\mathfrak{g}, \zeta} \in \neg \mathcal{T}_{\mathfrak{g}}} \neg \mathrm{op}_{\mathfrak{g}}\left(\mathcal{K}_{\mathfrak{g}, \zeta}\right) \quad \forall \xi \in \mathfrak{T}_{\mathfrak{g}} .
$$


Proof. Necessity. Let the $\mathcal{T}_{\mathfrak{g}}$-space $\mathfrak{T}_{\mathfrak{g}}$ be a $\mathfrak{g}-\mathcal{T}_{\mathfrak{g}}^{(\mathrm{H})}$-space $\mathfrak{g}$ - $\mathfrak{T}_{\mathfrak{g}}^{(\mathrm{H})}$. Then, for every $(\xi, \zeta) \in \mathfrak{g}-\mathrm{F}_{\mathrm{P}}[\sigma>0]$, there exists $\left(\mathcal{O}_{\mathfrak{g}, \xi}, \mathcal{O}_{\mathfrak{g}, \zeta}\right) \in \mathfrak{g}-\mathrm{F}_{\mathrm{O}}[\emptyset]$ such that:

$$
\left[\xi \in \mathrm{op}_{\mathfrak{g}}\left(\mathcal{O}_{\mathfrak{g}, \xi}\right)\right] \wedge\left[\zeta \in \mathrm{op}_{\mathfrak{g}}\left(\mathcal{O}_{\mathfrak{g}, \zeta}\right)\right]
$$

Consequently,

$$
\left[\xi \notin \complement\left(\mathrm{op}_{\mathfrak{g}}\left(\mathcal{O}_{\mathfrak{g}, \xi}\right)\right)\right] \wedge\left[\zeta \notin \complement\left(\mathrm{op}_{\mathfrak{g}}\left(\mathcal{O}_{\mathfrak{g}, \zeta}\right)\right)\right]
$$

But, for every $\lambda \in\{\xi, \zeta\}, \complement\left(\operatorname{op}_{\mathfrak{g}}\left(\mathcal{O}_{\mathfrak{g}, \lambda}\right)\right) \in \mathfrak{g}-\mathrm{K}\left[\mathfrak{T}_{\mathfrak{g}}\right]$ is a $\mathfrak{g}$ - $\mathfrak{T}_{\mathfrak{g}}$-closed set. Consequently, there exists $\left(\mathcal{K}_{\mathfrak{g}, \xi}, \mathcal{K}_{\mathfrak{g}, \zeta}\right) \in \neg \mathcal{T}_{\mathfrak{g}} \times \neg \mathcal{T}_{\mathfrak{g}}$ such that $\complement\left(\operatorname{op}_{\mathfrak{g}}\left(\mathcal{O}_{\mathfrak{g}, \xi}\right)\right) \supseteq \neg \mathrm{op}_{\mathfrak{g}}\left(\mathcal{K}_{\mathfrak{g}, \zeta}\right)$ and $\complement\left(\operatorname{op}_{\mathfrak{g}}\left(\mathcal{O}_{\mathfrak{g}, \zeta}\right)\right) \supseteq \neg \mathrm{op}_{\mathfrak{g}}\left(\mathcal{K}_{\mathfrak{g}, \xi}\right)$. Therefore, the relations $\xi \in \complement(\{\zeta\}) \supseteq \neg \mathrm{op}_{\mathfrak{g}}(\{\xi\})$ and $\zeta \in \complement(\{\xi\}) \supseteq \neg \mathrm{op}_{\mathfrak{g}}(\{\zeta\})$ are true for all $(\xi, \zeta) \in \mathfrak{T}_{\mathfrak{g}} \times \mathfrak{T}_{\mathfrak{g}}$. But, $\complement(\{\xi\})=$ $\bigcup_{\mathcal{O}_{\mathfrak{g}, \zeta} \in \mathcal{T}_{\mathfrak{g}}} \mathrm{op}_{\mathfrak{g}}\left(\mathcal{O}_{\mathfrak{g}, \zeta}\right)$ and hence,

$$
\{\xi\}=\complement(\complement(\{\xi\}))=\bigcap_{\mathcal{O}_{\mathfrak{g}, \zeta} \in \mathcal{T}_{\mathfrak{g}}} \complement\left(\mathrm{op}_{\mathfrak{g}}\left(\mathcal{O}_{\mathfrak{g}, \zeta}\right)\right)=\bigcap_{\mathcal{K}_{\mathfrak{g}, \zeta} \in \neg \mathcal{T}_{\mathfrak{g}}} \neg \mathrm{op}_{\mathfrak{g}}\left(\mathcal{K}_{\mathfrak{g}, \zeta}\right) \quad \forall \xi \in \mathfrak{T}_{\mathfrak{g}} .
$$

Sufficiency. Conversely, suppose $\{\xi\}=\bigcap_{\mathcal{K}_{\mathfrak{g}, \zeta} \in \neg \mathcal{T}_{\mathfrak{g}}} \neg \mathrm{op}_{\mathfrak{g}}\left(\mathcal{K}_{\mathfrak{g}, \zeta}\right)$ holds for all $\xi \in \mathfrak{T}_{\mathfrak{g}}$. Then, there exists a $\mathcal{K}_{\mathfrak{g}, \xi} \in \neg \mathcal{T}_{\mathfrak{g}}$ such that $\zeta \notin \neg \mathrm{op}_{\mathfrak{g}}\left(\mathcal{K}_{\mathfrak{g}, \xi}\right)$. Since $\neg \mathrm{op}_{\mathfrak{g}}\left(\mathcal{K}_{\mathfrak{g}, \xi}\right) \in$ $\mathfrak{g}-\mathrm{K}\left[\mathfrak{T}_{\mathfrak{g}}\right]$ is a $\mathfrak{g}-\mathfrak{T}_{\mathfrak{g}}$-closed set, there exists a $\mathfrak{g}-\mathfrak{T}_{\mathfrak{g}}$-open set op $\mathfrak{g}_{\mathfrak{g}}\left(\mathcal{O}_{\mathfrak{g}, \xi}\right) \in \mathfrak{g}-\mathrm{O}\left[\mathfrak{T}_{\mathfrak{g}}\right]$ such that $\xi \in \mathrm{op}_{\mathfrak{g}}\left(\mathcal{O}_{\mathfrak{g}, \xi}\right) \subseteq \neg \mathrm{op}_{\mathfrak{g}}\left(\mathcal{K}_{\mathfrak{g}, \xi}\right)$. But, since $\complement\left(\neg \mathrm{op}_{\mathfrak{g}}\left(\mathcal{K}_{\mathfrak{g}, \xi}\right)\right) \in \mathfrak{g}-\mathrm{O}\left[\mathfrak{T}_{\mathfrak{g}}\right]$ is a $\mathfrak{g}-\mathfrak{T}_{\mathfrak{g}}-$ open set containing $\zeta \in \mathfrak{T}_{\mathfrak{g}}$, it follows that $\mathrm{op}_{\mathfrak{g}}\left(\mathcal{O}_{\mathfrak{g}, \xi}\right), \complement\left(\neg \mathrm{op}_{\mathfrak{g}}\left(\mathcal{K}_{\mathfrak{g}, \xi}\right)\right) \in \mathfrak{g}-\mathrm{O}\left[\mathfrak{T}_{\mathfrak{g}}\right]$ are disjoint $\mathfrak{g}-\mathfrak{T}_{\mathfrak{g}}$-open sets. Thus, for every $(\xi, \zeta) \in \mathfrak{g}-\mathrm{F}_{\mathrm{P}}[\sigma>0]$, there exists $\left(\mathcal{O}_{\mathfrak{g}, \xi}, \mathcal{O}_{\mathfrak{g}, \zeta}\right) \in \mathfrak{g}-\mathrm{F}_{\mathrm{O}}[\emptyset]$ such that:

$$
\left[\xi \in \mathrm{op}_{\mathfrak{g}}\left(\mathcal{O}_{\mathfrak{g}, \xi)}\right) \wedge\left[\zeta \in \mathrm{op}_{\mathfrak{g}}\left(\mathcal{O}_{\mathfrak{g}, \zeta}\right)\right]\right.
$$

Therefore, $\mathfrak{T}_{\mathfrak{g}}=\left(\Omega, \mathcal{T}_{\mathfrak{g}}\right)$ is a $\mathfrak{g}-\mathcal{T}_{\mathfrak{g}}^{(\mathrm{H})}$-space $\mathfrak{g}-\mathfrak{T}_{\mathfrak{g}}^{(\mathrm{H})}=\left(\Omega, \mathfrak{g}-\mathcal{T}_{\mathfrak{g}}^{(\mathrm{H})}\right)$; this completes the proof of the theorem.

Q.E.D.

Proposition 3.5. If $\mathfrak{T}_{\mathfrak{g}}=\left(\Omega, \mathcal{T}_{\mathfrak{g}}\right)$ is a $\mathfrak{g}-\mathcal{T}_{\mathfrak{g}}^{(\mathrm{H})}$-space $\mathfrak{g}-\mathfrak{T}_{\mathfrak{g}}^{(\mathrm{H})}=\left(\Omega, \mathfrak{g}-\mathcal{T}_{\mathfrak{g}}^{(\mathrm{H})}\right)$, then it is a $\mathfrak{g}-\mathcal{T}_{\mathfrak{g}}^{(\mathrm{F})}$-space $\mathfrak{g}-\mathfrak{T}_{\mathfrak{g}}^{(\mathrm{F})}=\left(\Omega, \mathfrak{g}-\mathcal{T}_{\mathfrak{g}}^{(\mathrm{F})}\right)$.

Proof. Let $\mathfrak{T}_{\mathfrak{g}}=\left(\Omega, \mathcal{T}_{\mathfrak{g}}\right)$ be a $\mathfrak{g}-\mathcal{T}_{\mathfrak{g}}^{(\mathrm{H})}$-space $\mathfrak{g}-\mathfrak{T}_{\mathfrak{g}}^{(\mathrm{H})}=\left(\Omega, \mathfrak{g}-\mathcal{T}_{\mathfrak{g}}^{(\mathrm{H})}\right)$. Then, for every $(\xi, \zeta) \in \mathfrak{g}-\mathrm{F}_{\mathrm{P}}[\sigma>0]$, there exists a pair $\left(\mathcal{O}_{\mathfrak{g}, \xi}, \mathcal{O}_{\mathfrak{g}, \zeta}\right) \in \mathfrak{g}-\mathrm{F}_{\mathrm{O}}[\emptyset]$ such that $[\xi \in$ $\left.\operatorname{op}_{\mathfrak{g}}\left(\mathcal{O}_{\mathfrak{g}, \xi}\right)\right] \wedge\left[\zeta \in \operatorname{op}_{\mathfrak{g}}\left(\mathcal{O}_{\mathfrak{g}, \zeta}\right)\right]$. But since $\left(\mathcal{O}_{\mathfrak{g}, \xi}, \mathcal{O}_{\mathfrak{g}, \zeta}\right) \in \mathfrak{g}-\mathrm{F}_{\mathrm{O}}[\emptyset] \subset \mathfrak{g}-\mathrm{F}_{\mathrm{O}}\left[\mathcal{S}_{\mathfrak{g}} \supset \emptyset\right]$ and

$$
\begin{aligned}
{\left[\xi \in \mathrm{op}_{\mathfrak{g}}\left(\mathcal{O}_{\mathfrak{g}, \xi}\right)\right] } & \wedge\left[\zeta \in \mathrm{op}_{\mathfrak{g}}\left(\mathcal{O}_{\mathfrak{g}, \zeta}\right)\right] \\
\Leftrightarrow \quad\left[\left(\xi \in \mathrm{op}_{\mathfrak{g}}\left(\mathcal{O}_{\mathfrak{g}, \xi}\right)\right) \wedge\left(\zeta \in \mathrm{op}_{\mathfrak{g}}\left(\mathcal{O}_{\mathfrak{g}, \zeta}\right)\right)\right] & \wedge\left[\left(\xi \notin \mathrm{op}_{\mathfrak{g}}\left(\mathcal{O}_{\mathfrak{g}, \zeta}\right)\right)\right. \\
& \left.\wedge\left(\zeta \notin \mathrm{op}_{\mathfrak{g}}\left(\mathcal{O}_{\mathfrak{g}, \xi}\right)\right)\right],
\end{aligned}
$$

it follows that, if $\mathfrak{T}_{\mathfrak{g}}=\left(\Omega, \mathcal{T}_{\mathfrak{g}}\right)$ is a $\mathfrak{g}-\mathcal{T}_{\mathfrak{g}}^{(\mathrm{H})}$-space $\mathfrak{g}-\mathfrak{T}_{\mathfrak{g}}^{(\mathrm{H})}=\left(\Omega, \mathfrak{g}-\mathcal{T}_{\mathfrak{g}}^{(\mathrm{H})}\right)$, then for every $(\xi, \zeta) \in \mathfrak{g}-\mathrm{F}_{\mathrm{P}}[\sigma>0]$, there exists a pair $\left(\mathcal{O}_{\mathfrak{g}, \xi}, \mathcal{O}_{\mathfrak{g}, \zeta}\right) \in \mathfrak{g}-\mathrm{F}_{\mathrm{O}}\left[\mathcal{S}_{\mathfrak{g}} \supset \emptyset\right]$ such that:

$$
\left[(\xi, \zeta) \in \times_{\lambda=\xi, \zeta} \mathrm{op}_{\mathfrak{g}}\left(\mathcal{O}_{\mathfrak{g}, \lambda}\right)\right] \wedge\left[(\xi, \zeta) \notin \times_{\lambda=\zeta, \xi} \mathrm{op}_{\mathfrak{g}}\left(\mathcal{O}_{\mathfrak{g}, \lambda}\right)\right] .
$$

Hence, if $\mathfrak{T}_{\mathfrak{g}}=\left(\Omega, \mathcal{T}_{\mathfrak{g}}\right)$ is a $\mathfrak{g}-\mathcal{T}_{\mathfrak{g}}^{(\mathrm{H})}$-space $\mathfrak{g}-\mathfrak{T}_{\mathfrak{g}}^{(\mathrm{H})}=\left(\Omega, \mathfrak{g}-\mathcal{T}_{\mathfrak{g}}^{(\mathrm{H})}\right)$, then it is a $\mathfrak{g}-\mathcal{T}_{\mathfrak{g}}^{(\mathrm{F})}-$ space $\mathfrak{g}-\mathfrak{T}_{\mathfrak{g}}^{(\mathrm{F})}=\left(\Omega, \mathfrak{g}-\mathcal{T}_{\mathfrak{g}}^{(\mathrm{F})}\right)$.

Q.E.D. 
A necessary and sufficient condition for a $\mathcal{T}_{\mathfrak{g}}$-space $\mathfrak{T}_{\mathfrak{g}}=\left(\Omega, \mathcal{T}_{\mathfrak{g}}\right)$ to be a $\mathfrak{g}-\mathcal{T}_{\mathfrak{g}}^{(\mathrm{R})}$ space $\mathfrak{g}-\mathfrak{T}_{\mathfrak{g}}^{(\mathrm{R})}=\left(\Omega, \mathfrak{g}-\mathcal{T}_{\mathfrak{g}}^{(\mathrm{R})}\right)$ may be given in terms of the $\mathfrak{g}$-operator op $\mathfrak{g}: \mathcal{P}(\Omega) \rightarrow$ $\mathcal{P}(\Omega)$, a $\mathfrak{T}_{\mathfrak{g}}$-closed set $\mathcal{S}_{\mathfrak{g}} \subset \mathfrak{T}_{\mathfrak{g}}$, and $\mathcal{T}_{\mathfrak{g}}$-closed neighbourhood sets.

THEOREM 3.6. A $\mathcal{T}_{\mathfrak{g}}$-space $\mathfrak{T}_{\mathfrak{g}}=\left(\Omega, \mathcal{T}_{\mathfrak{g}}\right)$ is said to be a $\mathfrak{g}-\mathcal{T}_{\mathfrak{g}}^{(\mathrm{R})}$-space $\mathfrak{g}-\mathfrak{T}_{\mathfrak{g}}^{(\mathrm{R})}=$ $\left(\Omega, \mathfrak{g}-\mathcal{T}_{\mathfrak{g}}^{(\mathrm{R})}\right)$ if and only if the following condition holds:

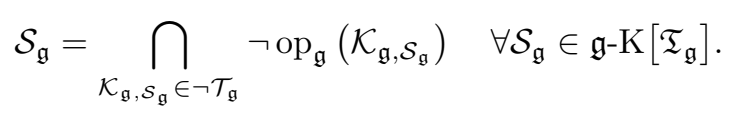

PRoOF. Necessity. Let the $\mathcal{T}_{\mathfrak{g}}$-space $\mathfrak{T}_{\mathfrak{g}}$ be a $\mathfrak{g}-\mathcal{T}_{\mathfrak{g}}^{(\mathrm{R})}$-space $\mathfrak{g}-\mathfrak{T}_{\mathfrak{g}}^{(\mathrm{R})}$. Then, for every $(\xi, \zeta) \in \mathfrak{g}-\mathrm{F}_{\mathrm{P}}[\sigma>0]$ and $\left(\mathcal{K}_{\mathfrak{g}, \xi}, \mathcal{K}_{\mathfrak{g}, \zeta}\right) \in \mathfrak{g}-\mathrm{F}_{\mathrm{K}}[\emptyset]$ such that $(\zeta, \xi) \notin\left(\mathcal{K}_{\mathfrak{g}, \xi}, \mathcal{K}_{\mathfrak{g}, \zeta}\right)$, there exists $\left(\mathcal{O}_{\mathfrak{g}, \xi}, \mathcal{O}_{\mathfrak{g}, \zeta}\right) \in \mathfrak{g}-\mathrm{F}_{\mathrm{O}}[\emptyset]$ such that:

$$
\begin{aligned}
{\left[\left(\neg \mathrm{op}_{\mathfrak{g}}\left(\mathcal{K}_{\mathfrak{g}, \xi}\right) \subset \mathrm{op}_{\mathfrak{g}}\left(\mathcal{O}_{\mathfrak{g}, \xi}\right)\right)\right.} & \left.\wedge\left(\zeta \in \mathrm{op}_{\mathfrak{g}}\left(\mathcal{O}_{\mathfrak{g}, \zeta}\right)\right)\right] \vee\left[\left(\neg \mathrm{op}_{\mathfrak{g}}\left(\mathcal{K}_{\mathfrak{g}, \zeta}\right)\right.\right. \\
& \left.\left.\subset \mathrm{op}_{\mathfrak{g}}\left(\mathcal{O}_{\mathfrak{g}, \zeta}\right)\right) \wedge\left(\xi \in \mathrm{op}_{\mathfrak{g}}\left(\mathcal{O}_{\mathfrak{g}, \xi}\right)\right)\right] .
\end{aligned}
$$

Consequently,

$$
\begin{aligned}
{\left[\left(\neg \mathrm{op}_{\mathfrak{g}}\left(\mathcal{K}_{\mathfrak{g}, \xi}\right) \subset \mathrm{op}_{\mathfrak{g}}\left(\mathcal{O}_{\mathfrak{g}, \xi}\right)\right)\right.} & \left.\wedge\left(\zeta \notin \complement\left(\mathrm{op}_{\mathfrak{g}}\left(\mathcal{O}_{\mathfrak{g}, \zeta}\right)\right)\right)\right] \vee\left[\left(\neg \mathrm{op}_{\mathfrak{g}}\left(\mathcal{K}_{\mathfrak{g}, \zeta}\right)\right.\right. \\
& \left.\left.\subset \mathrm{op}_{\mathfrak{g}}\left(\mathcal{O}_{\mathfrak{g}, \zeta}\right)\right) \wedge\left(\xi \notin \complement\left(\mathrm{op}_{\mathfrak{g}}\left(\mathcal{O}_{\mathfrak{g}, \xi}\right)\right)\right)\right] .
\end{aligned}
$$

But, for every $\lambda \in\{\xi, \zeta\}, \complement\left(\operatorname{op}_{\mathfrak{g}}\left(\mathcal{O}_{\mathfrak{g}, \lambda}\right)\right) \in \mathfrak{g}-\mathrm{K}\left[\mathfrak{T}_{\mathfrak{g}}\right]$ is a $\mathfrak{g}$ - $\mathfrak{T}_{\mathfrak{g}}$-closed set. Consequently, there exists $\left(\mathcal{K}_{\mathfrak{g}, \mathcal{R}_{\mathfrak{g}}}, \mathcal{K}_{\mathfrak{g}, \mathcal{S}_{\mathfrak{g}}}\right) \in \neg \mathcal{T}_{\mathfrak{g}} \times \neg \mathcal{T}_{\mathfrak{g}}$ such that $\complement\left(\mathrm{op}_{\mathfrak{g}}\left(\mathcal{O}_{\mathfrak{g}, \xi}\right)\right) \supseteq$ $\neg \mathrm{op}_{\mathfrak{g}}\left(\mathcal{K}_{\mathfrak{g}, \mathcal{R}_{\mathfrak{g}}}\right)$ and $\complement\left(\mathrm{op}_{\mathfrak{g}}\left(\mathcal{O}_{\mathfrak{g}, \zeta}\right)\right) \supseteq \neg \mathrm{op}_{\mathfrak{g}}\left(\mathcal{K}_{\mathfrak{g}, \mathcal{S}_{\mathfrak{g}}}\right)$. Therefore

$$
\begin{aligned}
& {\left[\left(\neg \mathrm{op}_{\mathfrak{g}}\left(\mathcal{K}_{\mathfrak{g}, \xi}\right) \subset \mathrm{op}_{\mathfrak{g}}\left(\mathcal{O}_{\mathfrak{g}, \xi}\right)\right) \wedge\left(\zeta \notin \neg \mathrm{op}_{\mathfrak{g}}\left(\mathcal{K}_{\mathfrak{g}, \mathcal{S}_{\mathfrak{g}}}\right)\right)\right] \vee\left[\left(\neg \mathrm{op}_{\mathfrak{g}}\left(\mathcal{K}_{\mathfrak{g}, \zeta}\right)\right.\right.} \\
& \left.\left.\subset \operatorname{op}_{\mathfrak{g}}\left(\mathcal{O}_{\mathfrak{g}, \zeta}\right)\right) \wedge\left(\xi \notin \neg \mathrm{op}_{\mathfrak{g}}\left(\mathcal{K}_{\mathfrak{g}, \mathcal{R}_{\mathfrak{g}}}\right)\right)\right] .
\end{aligned}
$$

By virtue of this logical statement, it consequently follows that

$$
\begin{aligned}
{\left[\left(\neg \mathrm{op}_{\mathfrak{g}}\left(\mathcal{K}_{\mathfrak{g}, \xi}\right) \subset \mathrm{op}_{\mathfrak{g}}\left(\mathcal{O}_{\mathfrak{g}, \xi}\right)\right)\right.} & \left.\wedge\left(\neg \mathrm{op}_{\mathfrak{g}}\left(\mathcal{K}_{\mathfrak{g}, \xi}\right) \supseteq \neg \mathrm{op}_{\mathfrak{g}}\left(\mathcal{K}_{\mathfrak{g}, \mathcal{S}_{\mathfrak{g}}}\right)\right)\right] \\
\vee\left[\left(\neg \mathrm{op}_{\mathfrak{g}}\left(\mathcal{K}_{\mathfrak{g}, \zeta}\right) \subset \mathrm{op}_{\mathfrak{g}}\left(\mathcal{O}_{\mathfrak{g}, \zeta}\right)\right)\right. & \left.\wedge\left(\neg \mathrm{op}_{\mathfrak{g}}\left(\mathcal{K}_{\mathfrak{g}, \xi}\right) \supseteq \neg \mathrm{op}_{\mathfrak{g}}\left(\mathcal{K}_{\mathfrak{g}, \mathcal{R}_{\mathfrak{g}}}\right)\right)\right],
\end{aligned}
$$

and, consequently,

$$
\begin{aligned}
& {\left[\neg \mathrm{op}_{\mathfrak{g}}\left(\mathcal{K}_{\mathfrak{g}, \mathcal{S}_{\mathfrak{g}}}\right) \subseteq \neg \mathrm{op}_{\mathfrak{g}}\left(\mathcal{K}_{\mathfrak{g}, \xi}\right) \subset \mathrm{op}_{\mathfrak{g}}\left(\mathcal{O}_{\mathfrak{g}, \xi}\right)\right]} \\
& \vee\left[\neg \mathrm{op}_{\mathfrak{g}}\left(\mathcal{K}_{\mathfrak{g}, \mathcal{R}_{\mathfrak{g}}}\right) \subseteq \neg \mathrm{op}_{\mathfrak{g}}\left(\mathcal{K}_{\mathfrak{g}, \zeta}\right) \subset \mathrm{op}_{\mathfrak{g}}\left(\mathcal{O}_{\mathfrak{g}, \zeta}\right)\right],
\end{aligned}
$$

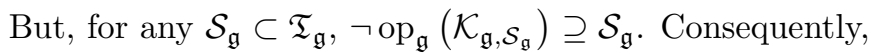

$$
\begin{gathered}
{\left[\mathcal{S}_{\mathfrak{g}} \subseteq \neg \mathrm{op}_{\mathfrak{g}}\left(\mathcal{K}_{\mathfrak{g}, \mathcal{S}_{\mathfrak{g}}}\right) \subseteq \neg \mathrm{op}_{\mathfrak{g}}\left(\mathcal{K}_{\mathfrak{g}, \xi}\right) \subset \mathrm{op}_{\mathfrak{g}}\left(\mathcal{O}_{\mathfrak{g}, \xi}\right)\right]} \\
\vee\left[\mathcal{R}_{\mathfrak{g}} \subseteq \neg \mathrm{op}_{\mathfrak{g}}\left(\mathcal{K}_{\mathfrak{g}, \mathcal{R}_{\mathfrak{g}}}\right) \subseteq \neg \mathrm{op}_{\mathfrak{g}}\left(\mathcal{K}_{\mathfrak{g}, \zeta}\right) \subset \mathrm{op}_{\mathfrak{g}}\left(\mathcal{O}_{\mathfrak{g}, \zeta}\right)\right],
\end{gathered}
$$

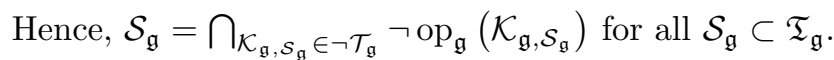

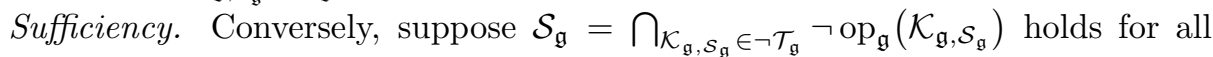
$\mathcal{S}_{\mathfrak{g}} \subset \mathfrak{T}_{\mathfrak{g}}$, let $\xi \notin \mathcal{S}_{\mathfrak{g}}$. Then, $\mathcal{S}_{\mathfrak{g}} \subseteq \neg \mathrm{op}_{\mathfrak{g}}\left(\mathcal{K}_{\left.\mathfrak{g}, \mathcal{S}_{\mathfrak{g}}\right)}\right)$ for every $\mathcal{T}_{\mathfrak{g}}$-closed neighbourhood set $\mathcal{K}_{\mathfrak{g}, \mathcal{S}_{\mathfrak{g}}} \in \neg \mathcal{T}_{\mathfrak{g}}$ satisfying $\mathcal{S}_{\mathfrak{g}} \subseteq \mathcal{K}_{\mathfrak{g}, \mathcal{S}_{\mathfrak{g}}}$. Therefore, there exists a $\mathcal{T}_{\mathfrak{g}}$-closed neighbourhood set $\mathcal{K}_{\mathfrak{g}, \mathcal{S}_{\mathfrak{g}}} \in \neg \mathcal{T}_{\mathfrak{g}}$ such that $\xi \notin \mathcal{K}_{\mathfrak{g}, \mathcal{S}_{\mathfrak{g}}}$. But, since $\mathcal{K}_{\mathfrak{g}, \mathcal{S}_{\mathfrak{g}}} \in \neg \mathcal{T}_{\mathfrak{g}}$ is a $\mathcal{T}_{\mathfrak{g}}$-closed neighbourhood set, there exists a $\mathcal{T}_{\mathfrak{g}}$-open set $\mathcal{O}_{\mathfrak{g}, \mathcal{S}_{\mathfrak{g}}} \in \mathcal{T}_{\mathfrak{g}}$ such that $\mathcal{S}_{\mathfrak{g}} \subset \mathrm{op}_{\mathfrak{g}}\left(\mathcal{O}_{\mathfrak{g}, \mathcal{S}_{\mathfrak{g}}}\right) \subset \neg \mathrm{op}_{\mathfrak{g}}\left(\mathcal{K}_{\mathfrak{g}, \mathcal{S}_{\mathfrak{g}}}\right)$, and $\complement\left(\mathcal{S}_{\mathfrak{g}}\right) \supset \complement\left(\mathrm{op}_{\mathfrak{g}}\left(\mathcal{O}_{\mathfrak{g}, \mathcal{S}_{\mathfrak{g}}}\right)\right) \supset \complement\left(\neg \mathrm{op}_{\mathfrak{g}}\left(\mathcal{K}_{\mathfrak{g}, \mathcal{S}_{\mathfrak{g}}}\right)\right)$. 
Because $\xi \in \complement\left(\neg \mathrm{op}_{\mathfrak{g}}\left(\mathcal{K}_{\mathfrak{g}, \mathcal{S}_{\mathfrak{g}}}\right)\right)$ and $\xi \in \complement\left(\mathrm{op}_{\mathfrak{g}}\left(\mathcal{O}_{\mathfrak{g}, \mathcal{S}_{\mathfrak{g}}}\right)\right)$, it follows that op $\mathrm{pp}_{\mathfrak{g}}\left(\mathcal{O}_{\mathfrak{g}, \mathcal{S}_{\mathfrak{g}}}\right) \cap$ $\complement\left(\neg \mathrm{op}_{\mathfrak{g}}\left(\mathcal{K}_{\mathfrak{g}, \mathcal{S}_{\mathfrak{g}}}\right)\right)=\emptyset$ and $\complement\left(\operatorname{op}_{\mathfrak{g}}\left(\mathcal{O}_{\mathfrak{g}, \mathcal{S}_{\mathfrak{g}}}\right)\right) \cap \neg \mathrm{op}_{\mathfrak{g}}\left(\mathcal{K}_{\mathfrak{g}, \mathcal{S}_{\mathfrak{g}}}\right)=\emptyset$, respectively. In other words, for every $(\xi, \zeta) \in \mathfrak{g}-\mathrm{F}_{\mathrm{P}}[\sigma>0]$ and $\left(\mathcal{K}_{\mathfrak{g}, \xi}, \mathcal{K}_{\mathfrak{g}, \zeta}\right) \in \mathfrak{g}-\mathrm{F}_{\mathrm{K}}[\emptyset]$ such that $(\zeta, \xi) \notin\left(\mathcal{K}_{\mathfrak{g}, \xi}, \mathcal{K}_{\mathfrak{g}, \zeta}\right)$, there exists $\left(\mathcal{O}_{\mathfrak{g}, \xi}, \mathcal{O}_{\mathfrak{g}, \zeta}\right) \in \mathfrak{g}-\mathrm{F}_{\mathrm{O}}[\emptyset]$ such that:

$$
\begin{aligned}
{\left[\left(\neg \mathrm{op}_{\mathfrak{g}}\left(\mathcal{K}_{\mathfrak{g}, \xi}\right) \subset \mathrm{op}_{\mathfrak{g}}\left(\mathcal{O}_{\mathfrak{g}, \xi}\right)\right)\right.} & \left.\wedge\left(\zeta \in \mathrm{op}_{\mathfrak{g}}\left(\mathcal{O}_{\mathfrak{g}, \zeta}\right)\right)\right] \vee\left[\left(\neg \mathrm{op}_{\mathfrak{g}}\left(\mathcal{K}_{\mathfrak{g}, \zeta}\right)\right.\right. \\
& \left.\left.\subset \mathrm{op}_{\mathfrak{g}}\left(\mathcal{O}_{\mathfrak{g}, \zeta}\right)\right) \wedge\left(\xi \in \mathrm{op}_{\mathfrak{g}}\left(\mathcal{O}_{\mathfrak{g}, \xi}\right)\right)\right] .
\end{aligned}
$$

Therefore, $\mathfrak{T}_{\mathfrak{g}}=\left(\Omega, \mathcal{T}_{\mathfrak{g}}\right)$ is a $\mathfrak{g}-\mathcal{T}_{\mathfrak{g}}^{(\mathrm{R})}$-space $\mathfrak{g}-\mathfrak{T}_{\mathfrak{g}}^{(\mathrm{R})}=\left(\Omega, \mathfrak{g}-\mathcal{T}_{\mathfrak{g}}^{(\mathrm{R})}\right)$; this completes the proof of the theorem.

Q.E.D.

PROPOSITION 3.7. If $\mathfrak{T}_{\mathfrak{g}}=\left(\Omega, \mathcal{T}_{\mathfrak{g}}\right)$ is a $\mathfrak{g}-\mathcal{T}_{\mathfrak{g}}^{(\mathrm{R})}$-space $\mathfrak{g}-\mathfrak{T}_{\mathfrak{g}}^{(\mathrm{R})}=\left(\Omega, \mathfrak{g}-\mathcal{T}_{\mathfrak{g}}^{(\mathrm{R})}\right)$, then it is a $\mathfrak{g}-\mathcal{T}_{\mathfrak{g}}^{(\mathrm{H})}$-space $\mathfrak{g}-\mathfrak{T}_{\mathfrak{g}}^{(\mathrm{H})}=\left(\Omega, \mathfrak{g}-\mathcal{T}_{\mathfrak{g}}^{(\mathrm{H})}\right)$.

PROOF. Let $\mathfrak{T}_{\mathfrak{g}}=\left(\Omega, \mathcal{T}_{\mathfrak{g}}\right)$ be a $\mathfrak{g}-\mathcal{T}_{\mathfrak{g}}^{(\mathrm{R})}$-space $\mathfrak{g}-\mathfrak{T}_{\mathfrak{g}}^{(\mathrm{R})}=\left(\Omega, \mathfrak{g}-\mathcal{T}_{\mathfrak{g}}^{(\mathrm{R})}\right)$. Then, for every $(\xi, \zeta) \in \mathfrak{g}-\mathrm{F}_{\mathrm{P}}[\sigma>0]$ and $\left(\mathcal{K}_{\mathfrak{g}, \xi}, \mathcal{K}_{\mathfrak{g}, \zeta}\right) \in \mathfrak{g}-\mathrm{F}_{\mathrm{K}}[\emptyset]$ such that $(\zeta, \xi) \notin\left(\mathcal{K}_{\mathfrak{g}, \xi}, \mathcal{K}_{\mathfrak{g}, \zeta}\right)$, there exists $\left(\mathcal{O}_{\mathfrak{g}, \xi}, \mathcal{O}_{\mathfrak{g}, \zeta}\right) \in \mathfrak{g}-\mathrm{F}_{\mathrm{O}}[\emptyset]$ such that:

$$
\begin{aligned}
{\left[\left(\neg \mathrm{op}_{\mathfrak{g}}\left(\mathcal{K}_{\mathfrak{g}, \xi}\right) \subset \mathrm{op}_{\mathfrak{g}}\left(\mathcal{O}_{\mathfrak{g}, \xi}\right)\right)\right.} & \left.\wedge\left(\zeta \in \mathrm{op}_{\mathfrak{g}}\left(\mathcal{O}_{\mathfrak{g}, \zeta}\right)\right)\right] \vee\left[\left(\neg \mathrm{op}_{\mathfrak{g}}\left(\mathcal{K}_{\mathfrak{g}, \zeta}\right)\right.\right. \\
& \left.\left.\subset \mathrm{op}_{\mathfrak{g}}\left(\mathcal{O}_{\mathfrak{g}, \zeta}\right)\right) \wedge\left(\xi \in \mathrm{op}_{\mathfrak{g}}\left(\mathcal{O}_{\mathfrak{g}, \xi}\right)\right)\right] .
\end{aligned}
$$

Set $\mathrm{Q}(\xi)=\left(\neg \mathrm{op}_{\mathfrak{g}}\left(\mathcal{K}_{\mathfrak{g}, \xi}\right) \subset \mathrm{op}_{\mathfrak{g}}\left(\mathcal{O}_{\mathfrak{g}, \xi}\right)\right), \mathrm{R}(\zeta)=\left(\zeta \in \mathrm{op}_{\mathfrak{g}}\left(\mathcal{O}_{\mathfrak{g}, \zeta}\right)\right)$, and $\mathrm{P}(\xi, \zeta)=$ $\mathrm{Q}(\xi) \wedge \mathrm{R}(\zeta)$. Then, the above logical statement is equivalent to $\mathrm{P}(\xi, \zeta) \vee \mathrm{P}(\zeta, \xi)$. But since $\lambda \in \neg \mathrm{op}_{\mathfrak{g}}\left(\mathcal{K}_{\mathfrak{g}, \lambda}\right)$ for every $\lambda\{\xi, \zeta\}$, it consequently follows that $\mathrm{R}(\lambda) \leftarrow$ $\mathrm{Q}(\lambda)$ for every $\lambda\{\xi, \zeta\}$. Therefore $\mathrm{R}(\xi) \wedge \mathrm{R}(\zeta) \leftarrow \mathrm{P}(\xi, \zeta)$. Because associativity with respect to $\wedge$ holds, it then follows that

$$
\mathrm{P}(\xi, \zeta) \vee \mathrm{P}(\zeta, \xi) \rightarrow[\mathrm{R}(\xi) \wedge \mathrm{R}(\zeta)] \vee[\mathrm{R}(\zeta) \wedge \mathrm{R}(\xi)]=\mathrm{R}(\xi) \wedge \mathrm{R}(\zeta)
$$

Hence, for every $(\xi, \zeta) \in \mathfrak{g}-\mathrm{F}_{\mathrm{P}}[\sigma>0]$, there exists $\left(\mathcal{O}_{\mathfrak{g}, \xi}, \mathcal{O}_{\mathfrak{g}, \zeta}\right) \in \mathfrak{g}-\mathrm{F}_{\mathrm{O}}[\emptyset]$ such that:

$$
\left[\xi \in \mathrm{op}_{\mathfrak{g}}\left(\mathcal{O}_{\mathfrak{g}, \xi}\right)\right] \wedge\left[\zeta \in \mathrm{op}_{\mathfrak{g}}\left(\mathcal{O}_{\mathfrak{g}, \zeta}\right)\right]
$$

This proves that, if $\mathfrak{T}_{\mathfrak{g}}=\left(\Omega, \mathcal{T}_{\mathfrak{g}}\right)$ is a $\mathfrak{g}-\mathcal{T}_{\mathfrak{g}}^{(\mathrm{R})}$-space $\mathfrak{g}-\mathfrak{T}_{\mathfrak{g}}^{(\mathrm{R})}=\left(\Omega, \mathfrak{g}-\mathcal{T}_{\mathfrak{g}}^{(\mathrm{R})}\right)$, then it is a $\mathfrak{g}-\mathcal{T}_{\mathfrak{g}}^{(\mathrm{H})}$-space $\mathfrak{g}-\mathfrak{T}_{\mathfrak{g}}^{(\mathrm{H})}=\left(\Omega, \mathfrak{g}-\mathcal{T}_{\mathfrak{g}}^{(\mathrm{H})}\right)$.

Q.E.D.

A necessary and sufficient condition for a $\mathcal{T}_{\mathfrak{g}}$-space $\mathfrak{T}_{\mathfrak{g}}=\left(\Omega, \mathcal{T}_{\mathfrak{g}}\right)$ to be a $\mathfrak{g}-\mathcal{T}_{\mathfrak{g}}^{(\mathrm{N})}$ space $\mathfrak{g}-\mathfrak{T}_{\mathfrak{g}}^{(\mathrm{N})}=\left(\Omega, \mathfrak{g}-\mathcal{T}_{\mathfrak{g}}^{(\mathrm{N})}\right)$ may be given in terms of the $\mathfrak{g}$-operator op $\mathfrak{g}: \mathcal{P}(\Omega) \rightarrow$ $\mathcal{P}(\Omega)$, a $\mathfrak{T}_{\mathfrak{g}}$-closed set $\mathcal{S}_{\mathfrak{g}} \subset \mathfrak{T}_{\mathfrak{g}}, \mathcal{T}_{\mathfrak{g}}$-open sets, and a $\mathcal{T}_{\mathfrak{g}}$-closed set.

TheOREM 3.8. A $\mathcal{T}_{\mathfrak{g}}$-space $\mathfrak{T}_{\mathfrak{g}}=\left(\Omega, \mathcal{T}_{\mathfrak{g}}\right)$ is said to be a $\mathfrak{g}-\mathcal{T}_{\mathfrak{g}}^{(\mathrm{N})}$-space $\mathfrak{g}-\mathfrak{T}_{\mathfrak{g}}^{(\mathrm{N})}=$ $\left(\Omega, \mathfrak{g}-\mathcal{T}_{\mathfrak{g}}^{(\mathrm{N})}\right)$ if and only if the following condition holds:

$$
\mathcal{S}_{\mathfrak{g}} \subset \mathrm{op}_{\mathfrak{g}}\left(\hat{\mathcal{O}}_{\mathfrak{g}, \mathcal{S}_{\mathfrak{g}}}\right) \subset \neg \mathrm{op}_{\mathfrak{g}}\left(\hat{\mathcal{K}}_{\mathfrak{g}, \mathcal{S}_{\mathfrak{g}}}\right) \subset \mathrm{op}_{\mathfrak{g}}\left(\mathcal{O}_{\mathfrak{g}, \mathcal{S}_{\mathfrak{g}}}\right) \quad \forall \mathcal{S}_{\mathfrak{g}} \in \mathfrak{g}-\mathrm{K}\left[\mathfrak{T}_{\mathfrak{g}}\right] .
$$

Proof. Necessity. Let the $\mathcal{T}_{\mathfrak{g}}$-space $\mathfrak{T}_{\mathfrak{g}}$ be a $\mathfrak{g}$ - $\mathcal{T}_{\mathfrak{g}}^{(\mathrm{N})}$-space $\mathfrak{g}$ - $\mathfrak{T}_{\mathfrak{g}}^{(\mathrm{N})}$ and, let $\mathcal{S}_{\mathfrak{g}} \in$ $\mathfrak{g}-\mathrm{K}\left[\mathfrak{T}_{\mathfrak{g}}\right]$ and $\mathcal{O}_{\mathfrak{g}, \mathcal{S}_{\mathfrak{g}}} \in \mathcal{T}_{\mathfrak{g}}$, respectively, be a $\mathfrak{g}-\mathfrak{T}_{\mathfrak{g}}$-closed set and a $\mathcal{T}_{\mathfrak{g}}$-open neighbourhood set of $\mathcal{S}_{\mathfrak{g}}$. Then, $\mathfrak{T}_{\mathfrak{g}}$ is a $\mathfrak{g}-\mathcal{T}_{\mathfrak{g}}^{(\mathrm{N})}$-space $\mathfrak{g}-\mathfrak{T}_{\mathfrak{g}}^{(\mathrm{N})}$ implies that, for every $\left(\mathcal{K}_{\mathfrak{g}, \xi}, \mathcal{K}_{\mathfrak{g}, \zeta}\right) \in \mathfrak{g}-\mathrm{F}_{\mathrm{K}}[\emptyset]$, there exists $\left(\mathcal{O}_{\mathfrak{g}, \xi}, \mathcal{O}_{\mathfrak{g}, \zeta}\right) \in \mathfrak{g}-\mathrm{F}_{\mathrm{O}}[\emptyset]$ such that:

$$
\left[\operatorname{op}_{\mathfrak{g}}\left(\mathcal{O}_{\mathfrak{g}, \xi}\right) \supset \neg \mathrm{op}_{\mathfrak{g}}\left(\mathcal{K}_{\mathfrak{g}, \xi}\right)\right] \wedge\left[\mathrm{op}_{\mathfrak{g}}\left(\mathcal{O}_{\mathfrak{g}, \zeta}\right) \supset \neg \mathrm{op}_{\mathfrak{g}}\left(\mathcal{K}_{\mathfrak{g}, \zeta}\right)\right] .
$$


Clearly, $\complement\left(\operatorname{op}_{\mathfrak{g}}\left(\mathcal{O}_{\mathfrak{g}, \xi}\right)\right) \cap \neg \mathrm{op}_{\mathfrak{g}}\left(\mathcal{K}_{\mathfrak{g}, \xi}\right)=\emptyset$ for any $\xi \in \mathfrak{T}_{\mathfrak{g}}$. The relation $\mathcal{S}_{\mathfrak{g}} \in \mathfrak{g}-\mathrm{K}\left[\mathfrak{T}_{\mathfrak{g}}\right]$ implies that there exists a $\hat{\mathcal{K}}_{\mathfrak{g}, \xi} \in \neg \mathcal{T}_{\mathfrak{g}}$ such that $\mathcal{S}_{\mathfrak{g}} \supseteq \neg \mathrm{op}_{\mathfrak{g}}\left(\hat{\mathcal{K}}_{\mathfrak{g}, \xi}\right)$ and, $\mathcal{O}_{\mathfrak{g}, \mathcal{S}_{\mathfrak{g}}} \in \mathcal{T}_{\mathfrak{g}}$ is a $\mathcal{T}_{\mathfrak{g}}$-open neighbourhood set of $\mathcal{S}_{\mathfrak{g}}$ implies that there exists $\left(\hat{\mathcal{O}}_{\mathfrak{g}, \mathcal{S}_{\mathfrak{g}}}, \hat{\mathcal{K}}_{\mathfrak{g}, \mathcal{S}_{\mathfrak{g}}}\right) \in$ $\mathcal{T}_{\mathfrak{g}} \times \neg \mathcal{T}_{\mathfrak{g}}$ such that $\mathcal{O}_{\mathfrak{g}, \mathcal{S}_{\mathfrak{g}}} \subseteq \mathrm{op}_{\mathfrak{g}}\left(\hat{\mathcal{O}}_{\mathfrak{g}, \mathcal{S}_{\mathfrak{g}}}\right) \subset \neg \mathrm{op}_{\mathfrak{g}}\left(\hat{\mathcal{K}}_{\mathfrak{g}, \mathcal{S}_{\mathfrak{g}}}\right)$. But, $\mathcal{S}_{\mathfrak{g}} \subset \mathcal{O}_{\mathfrak{g}, \mathcal{S}_{\mathfrak{g}}}$ and, for some $\hat{\mathcal{O}}_{\mathfrak{g}, \xi} \in \neg \mathcal{T}_{\mathfrak{g}}$, the relation $\neg \mathrm{op}_{\mathfrak{g}}\left(\hat{\mathcal{K}}_{\mathfrak{g}, \mathcal{S}_{\mathfrak{g}}}\right) \subset \operatorname{op}_{\mathfrak{g}}\left(\hat{\mathcal{O}}_{\mathfrak{g}, \xi}\right)$ holds in a $\mathfrak{g}-\mathcal{T}_{\mathfrak{g}}^{(\mathrm{N})}$-space $\mathfrak{g}-\mathfrak{T}_{\mathfrak{g}}^{(\mathrm{N})}$. Hence,

$$
\neg \mathrm{op}_{\mathfrak{g}}\left(\hat{\mathcal{K}}_{\mathfrak{g}, \xi}\right) \subseteq \mathcal{S}_{\mathfrak{g}} \subset \mathrm{op}_{\mathfrak{g}}\left(\hat{\mathcal{O}}_{\mathfrak{g}, \mathcal{S}_{\mathfrak{g}}}\right) \subset \neg \mathrm{op}_{\mathfrak{g}}\left(\hat{\mathcal{K}}_{\mathfrak{g}, \mathcal{S}_{\mathfrak{g}}}\right) \subset \mathrm{op}_{\mathfrak{g}}\left(\hat{\mathcal{O}}_{\mathfrak{g}, \xi}\right)
$$

for all $\mathcal{S}_{\mathfrak{g}} \in \mathfrak{g}-\mathrm{K}\left[\mathfrak{T}_{\mathfrak{g}}\right]$. At this stage, it suffices to set $\hat{\mathcal{O}}_{\mathfrak{g}, \xi} \subseteq \mathcal{O}_{\mathfrak{g}, \mathcal{S}_{\mathfrak{g}}}$ and the result follows.

Sufficiency. Conversely, suppose the following relation holds:

$$
\mathcal{S}_{\mathfrak{g}} \subset \operatorname{op}_{\mathfrak{g}}\left(\hat{\mathcal{O}}_{\mathfrak{g}, \mathcal{S}_{\mathfrak{g}}}\right) \subset \neg \mathrm{op}_{\mathfrak{g}}\left(\hat{\mathcal{K}}_{\mathfrak{g}, \mathcal{S}_{\mathfrak{g}}}\right) \subset \mathrm{op}_{\mathfrak{g}}\left(\mathcal{O}_{\mathfrak{g}, \mathcal{S}_{\mathfrak{g}}}\right) \quad \forall \mathcal{S}_{\mathfrak{g}} \in \mathfrak{g}-\mathrm{K}\left[\mathfrak{T}_{\mathfrak{g}}\right]
$$

Then, its complementary reads

$$
\complement\left(\mathcal{S}_{\mathfrak{g}}\right) \supset \complement\left(\mathrm{op}_{\mathfrak{g}}\left(\hat{\mathcal{O}}_{\mathfrak{g}, \mathcal{S}_{\mathfrak{g}}}\right)\right) \supset \complement\left(\neg \mathrm{op}_{\mathfrak{g}}\left(\hat{\mathcal{K}}_{\mathfrak{g}, \mathcal{S}_{\mathfrak{g}}}\right)\right) \supset \complement\left(\mathrm{op}_{\mathfrak{g}}\left(\mathcal{O}_{\mathfrak{g}, \mathcal{S}_{\mathfrak{g}}}\right)\right),
$$

where $\complement\left(\mathcal{S}_{\mathfrak{g}}\right), \complement\left(\neg \mathrm{op}_{\mathfrak{g}}\left(\hat{\mathcal{K}}_{\mathfrak{g}, \mathcal{S}_{\mathfrak{g}}}\right)\right) \in \mathfrak{g}-\mathrm{O}\left[\mathfrak{T}_{\mathfrak{g}}\right]$ are $\mathfrak{g}-\mathfrak{T}_{\mathfrak{g}}$-open sets and, $\complement\left(\mathrm{op}_{\mathfrak{g}}\left(\hat{\mathcal{O}}_{\mathfrak{g}, \mathcal{S}_{\mathfrak{g}}}\right)\right)$, $\complement\left(\operatorname{op}_{\mathfrak{g}}\left(\mathcal{O}_{\mathfrak{g}, \mathcal{S}_{\mathfrak{g}}}\right)\right) \in \mathfrak{g}-\mathrm{K}\left[\mathfrak{T}_{\mathfrak{g}}\right]$ are $\mathfrak{g}$ - $\mathfrak{T}_{\mathfrak{g}}$-closed sets. Thus, $\mathcal{S}_{\mathfrak{g}} \cap \complement\left(\operatorname{op}_{\mathfrak{g}}\left(\mathcal{O}_{\mathfrak{g}, \mathcal{S}_{\mathfrak{g}}}\right)\right)=\emptyset$ for any $\mathcal{S}_{\mathfrak{g}} \in \mathfrak{g}-\mathrm{K}\left[\mathfrak{T}_{\mathfrak{g}}\right]$. But since the relation $\mathcal{S}_{\mathfrak{g}} \supseteq \neg \mathrm{op}_{\mathfrak{g}}\left(\mathcal{K}_{\mathfrak{g}, \xi}\right)$ holds for some $\mathcal{K}_{\mathfrak{g}, \xi} \in$ $\neg \mathcal{T}_{\mathfrak{g}}$, it consequently follows that $\complement\left(\operatorname{op}_{\mathfrak{g}}\left(\mathcal{O}_{\mathfrak{g}, \mathcal{S}_{\mathfrak{g}}}\right)\right) \subset \complement\left(\mathcal{S}_{\mathfrak{g}}\right) \subseteq \complement\left(\neg \mathrm{op}_{\mathfrak{g}}\left(\mathcal{K}_{\mathfrak{g}, \xi}\right)\right)$ which, in turn, implies op $\operatorname{gos}_{\mathfrak{g}}\left(\mathcal{O}_{\mathfrak{g}, \mathcal{S}_{\mathfrak{g}}}\right) \supset \mathcal{S}_{\mathfrak{g}} \supseteq \neg \operatorname{op}_{\mathfrak{g}}\left(\mathcal{K}_{\mathfrak{g}, \xi}\right)$. Thus, for every $\left(\mathcal{K}_{\mathfrak{g}, \xi}, \mathcal{K}_{\mathfrak{g}, \zeta}\right) \in$ $\mathfrak{g}-\mathrm{F}_{\mathrm{K}}[\emptyset]$, there exists $\left(\mathcal{O}_{\mathfrak{g}, \xi}, \mathcal{O}_{\mathfrak{g}, \zeta}\right) \in \mathfrak{g}-\mathrm{F}_{\mathrm{O}}[\emptyset]$ such that:

$$
\left[\operatorname{op}_{\mathfrak{g}}\left(\mathcal{O}_{\mathfrak{g}, \xi}\right) \supset \neg \mathrm{op}_{\mathfrak{g}}\left(\mathcal{K}_{\mathfrak{g}, \xi}\right)\right] \wedge\left[\operatorname{op}_{\mathfrak{g}}\left(\mathcal{O}_{\mathfrak{g}, \zeta}\right) \supset \neg \mathrm{op}_{\mathfrak{g}}\left(\mathcal{K}_{\mathfrak{g}, \zeta}\right)\right] .
$$

Therefore, $\mathfrak{T}_{\mathfrak{g}}=\left(\Omega, \mathcal{T}_{\mathfrak{g}}\right)$ is a $\mathfrak{g}-\mathcal{T}_{\mathfrak{g}}^{(\mathrm{N})}$-space $\mathfrak{g}-\mathfrak{T}_{\mathfrak{g}}^{(\mathrm{N})}=\left(\Omega, \mathfrak{g}-\mathcal{T}_{\mathfrak{g}}^{(\mathrm{N})}\right)$; this completes the proof of the theorem.

Q.E.D.

Proposition 3.9. If $\mathfrak{T}_{\mathfrak{g}}=\left(\Omega, \mathcal{T}_{\mathfrak{g}}\right)$ is a $\mathfrak{g}-\mathcal{T}_{\mathfrak{g}}^{(\mathrm{N})}$-space $\mathfrak{g}-\mathfrak{T}_{\mathfrak{g}}^{(\mathrm{N})}=\left(\Omega, \mathfrak{g}-\mathcal{T}_{\mathfrak{g}}^{(\mathrm{N})}\right)$, then it is a $\mathfrak{g}-\mathcal{T}_{\mathfrak{g}}^{(\mathrm{R})}$-space $\mathfrak{g}-\mathfrak{T}_{\mathfrak{g}}^{(\mathrm{R})}=\left(\Omega, \mathfrak{g}-\mathcal{T}_{\mathfrak{g}}^{(\mathrm{R})}\right)$.

Proof. Let $\mathfrak{T}_{\mathfrak{g}}=\left(\Omega, \mathcal{T}_{\mathfrak{g}}\right)$ be a $\mathfrak{g}-\mathcal{T}_{\mathfrak{g}}^{(\mathrm{N})}$-space $\mathfrak{g}-\mathfrak{T}_{\mathfrak{g}}^{(\mathrm{N})}=\left(\Omega, \mathfrak{g}-\mathcal{T}_{\mathfrak{g}}^{(\mathrm{N})}\right)$. Then, for every $\left(\mathcal{K}_{\mathfrak{g}, \xi}, \mathcal{K}_{\mathfrak{g}, \zeta}\right) \in \mathfrak{g}-\mathrm{F}_{\mathrm{K}}[\emptyset]$, there exists $\left(\mathcal{O}_{\mathfrak{g}, \xi}, \mathcal{O}_{\mathfrak{g}, \zeta}\right) \stackrel{\mathfrak{g}}{\in} \mathfrak{g}-\mathrm{F}_{\mathrm{O}}[\emptyset]$ such that:

$$
\left[\mathrm{op}_{\mathfrak{g}}\left(\mathcal{O}_{\mathfrak{g}, \xi}\right) \supset \neg \mathrm{op}_{\mathfrak{g}}\left(\mathcal{K}_{\mathfrak{g}, \xi}\right)\right] \wedge\left[\mathrm{op}_{\mathfrak{g}}\left(\mathcal{O}_{\mathfrak{g}, \zeta}\right) \supset \neg \mathrm{op}_{\mathfrak{g}}\left(\mathcal{K}_{\mathfrak{g}, \zeta}\right)\right] .
$$

Set $\mathrm{Q}(\xi)=\left(\neg \mathrm{op}_{\mathfrak{g}}\left(\mathcal{K}_{\mathfrak{g}, \xi}\right) \subset \mathrm{op}_{\mathfrak{g}}\left(\mathcal{O}_{\mathfrak{g}, \xi}\right)\right)$ and $\mathrm{R}(\xi)=\left(\xi \in \mathrm{op}_{\mathfrak{g}}\left(\mathcal{O}_{\mathfrak{g}, \xi}\right)\right)$ so that the above logical statement now reads $\mathrm{Q}(\xi) \wedge \mathrm{Q}(\zeta)$. Then, since $\left(\mathcal{K}_{\mathfrak{g}, \xi}, \mathcal{K}_{\mathfrak{g}, \zeta}\right) \in \mathfrak{g}-\mathrm{F}_{\mathrm{K}}[\emptyset]$, $\mathrm{Q}(\xi) \wedge \mathrm{R}(\zeta) \leftarrow \mathrm{Q}(\xi)$ and $\mathrm{Q}(\zeta) \wedge \mathrm{R}(\xi) \leftarrow \mathrm{Q}(\zeta)$ hold. Consequently,

$$
[\mathrm{Q}(\xi) \wedge \mathrm{R}(\zeta)] \wedge[\mathrm{Q}(\zeta) \wedge \mathrm{R}(\xi)] \leftarrow \mathrm{Q}(\xi) \wedge \mathrm{Q}(\zeta) .
$$

But

$$
[\mathrm{Q}(\xi) \wedge \mathrm{R}(\zeta)] \vee[\mathrm{Q}(\zeta) \wedge \mathrm{R}(\xi)] \leftarrow[\mathrm{Q}(\xi) \wedge \mathrm{R}(\zeta)] \wedge[\mathrm{Q}(\zeta) \wedge \mathrm{R}(\xi)]
$$

and, therefore,

$$
[\mathrm{Q}(\xi) \wedge \mathrm{R}(\zeta)] \vee[\mathrm{Q}(\zeta) \wedge \mathrm{R}(\xi)] \leftarrow \mathrm{Q}(\xi) \wedge \mathrm{Q}(\zeta)
$$


Thus, for every $(\xi, \zeta) \in \mathfrak{g}-\mathrm{F}_{\mathrm{P}}[\sigma>0]$ and $\left(\mathcal{K}_{\mathfrak{g}, \xi}, \mathcal{K}_{\mathfrak{g}, \zeta}\right) \in \mathfrak{g}-\mathrm{F}_{\mathrm{K}}[\emptyset]$ such that $(\zeta, \xi) \notin$ $\left(\mathcal{K}_{\mathfrak{g}, \xi}, \mathcal{K}_{\mathfrak{g}, \zeta}\right)$, there exists $\left(\mathcal{O}_{\mathfrak{g}, \xi}, \mathcal{O}_{\mathfrak{g}, \zeta}\right) \in \mathfrak{g}-\mathrm{F}_{\mathrm{O}}[\emptyset]$ such that:

$$
\begin{aligned}
{\left[\left(\neg \mathrm{op}_{\mathfrak{g}}\left(\mathcal{K}_{\mathfrak{g}, \xi}\right) \subset \mathrm{op}_{\mathfrak{g}}\left(\mathcal{O}_{\mathfrak{g}, \xi}\right)\right)\right.} & \left.\wedge\left(\zeta \in \mathrm{op}_{\mathfrak{g}}\left(\mathcal{O}_{\mathfrak{g}, \zeta}\right)\right)\right] \vee\left[\left(\neg \mathrm{op}_{\mathfrak{g}}\left(\mathcal{K}_{\mathfrak{g}, \zeta}\right)\right.\right. \\
& \left.\left.\subset \mathrm{op}_{\mathfrak{g}}\left(\mathcal{O}_{\mathfrak{g}, \zeta}\right)\right) \wedge\left(\xi \in \mathrm{op}_{\mathfrak{g}}\left(\mathcal{O}_{\mathfrak{g}, \xi}\right)\right)\right] .
\end{aligned}
$$

This proves that, if $\mathfrak{T}_{\mathfrak{g}}=\left(\Omega, \mathcal{T}_{\mathfrak{g}}\right)$ is a $\mathfrak{g}-\mathcal{T}_{\mathfrak{g}}^{(\mathrm{N})}$-space $\mathfrak{g}-\mathfrak{T}_{\mathfrak{g}}^{(\mathrm{N})}=\left(\Omega, \mathfrak{g}-\mathcal{T}_{\mathfrak{g}}^{(\mathrm{N})}\right)$, then it is a $\mathfrak{g}-\mathcal{T}_{\mathfrak{g}}^{(\mathrm{R})}$-space $\mathfrak{g}-\mathfrak{T}_{\mathfrak{g}}^{(\mathrm{R})}=\left(\Omega, \mathfrak{g}-\mathcal{T}_{\mathfrak{g}}^{(\mathrm{R})}\right)$.

Q.E.D.

By virtue of the above propositions, every $\mathfrak{g}-\mathcal{T}_{\mathfrak{g}}^{(\mathrm{H})}$-space is a $\mathfrak{g}-\mathcal{T}_{\mathfrak{g}}^{(\mathrm{F})}$-space, and hence, a $\mathfrak{g}-\mathcal{T}_{\mathfrak{g}}^{(\mathrm{K})}$-space. Also, every $\mathfrak{g}-\mathcal{T}_{\mathfrak{g}}^{(\mathrm{N})}$-space is a $\mathfrak{g}-\mathcal{T}_{\mathfrak{g}}^{(\mathrm{R})}$-space, and hence, a $\mathfrak{g}-\mathcal{T}_{\mathfrak{g}}^{(\mathrm{H})}$-space. But, the converse of both statements are untrue, and thus, the corollary follows.

Corollary 3.10. If $\left\langle\mathfrak{g}-\mathfrak{T}_{\mathfrak{g}}^{(\alpha)}=\left(\Omega, \mathfrak{g}-\mathcal{T}_{\mathfrak{g}}^{(\alpha)}\right)\right\rangle_{\alpha \in \Lambda}, \Lambda=\{\mathrm{K}, \mathrm{F}, \mathrm{H}, \mathrm{R}, \mathrm{N}\}$, denotes a sequence of $\mathfrak{g}-\mathcal{T}_{\mathfrak{g}}^{(\alpha)}$-spaces, obtained after endowing a $\mathcal{T}_{\mathfrak{g}}$-space $\mathfrak{T}_{\mathfrak{g}}=\left(\Omega, \mathcal{T}_{\mathfrak{g}}\right)$ with the sequence of $\mathfrak{g}-\mathrm{T}_{\mathfrak{g}, \alpha}$-axioms $\left\langle\mathfrak{g}-\mathrm{T}_{\mathfrak{g}, \alpha}\right\rangle_{\alpha \in \Lambda}$, then the following relations hold:

- I. $\mathfrak{T}_{\mathfrak{g}}^{(\mathrm{K})} \subseteq \mathfrak{T}_{\mathfrak{g}}^{(\mathrm{F})} \subseteq \mathfrak{T}_{\mathfrak{g}}^{(\mathrm{H})} \subseteq \mathfrak{T}_{\mathfrak{g}}^{(\mathrm{R})} \subseteq \mathfrak{T}_{\mathfrak{g}}^{(\mathrm{N})}$.

- II. $\mathfrak{g}-\mathrm{T}_{\mathfrak{g}, \mathrm{N}} \Rightarrow \mathfrak{g}-\mathrm{T}_{\mathfrak{g}, \mathrm{R}} \Rightarrow \mathfrak{g}-\mathrm{T}_{\mathfrak{g}, \mathrm{H}} \Rightarrow \mathfrak{g}-\mathrm{T}_{\mathfrak{g}, \mathrm{F}} \Rightarrow \mathfrak{g}-\mathrm{T}_{\mathfrak{g}, \mathrm{K}}$.

\section{Discussion}

4.1. Categorical Classifications. Having adopted a categorical approach in the classifications of the $\mathfrak{g}$ - $T_{\mathfrak{g}, \alpha}$-axioms, $\alpha \in \Lambda=\{\mathrm{K}, \mathrm{F}, \mathrm{H}, \mathrm{R}, \mathrm{N}\}$, in the $\mathcal{T}_{\mathfrak{g}}$-space $\mathfrak{T}_{\mathfrak{g}}$, the aims here are, to establish the various relationships amongst the elements of the sequence $\left\langle\mathfrak{g}-\mathrm{T}_{\mathfrak{g}, \alpha}\right\rangle_{\alpha \in \Lambda}$ and, to illustrate them through diagrams.

We have seen that, both the $\mathfrak{g}-T_{\mathfrak{g}, N}, \mathfrak{g}-T_{\mathfrak{g}, R}$-axioms imply the $\mathfrak{g}-T_{\mathfrak{g}, K}, \mathfrak{g}-T_{\mathfrak{g}, F^{-}}$ axioms and, on the other hand, the $\mathfrak{g}-T_{\mathfrak{g}, \mathrm{N}}$-axiom implies the $\mathfrak{g}-\mathrm{T}_{\mathfrak{g}, \mathrm{R}}$-axiom and the $\mathfrak{g}-\mathrm{T}_{\mathfrak{g}, \mathrm{H}}$-axiom implies the $\mathfrak{g}-\mathrm{T}_{\mathfrak{g}, \mathrm{F}}$-axioms. The separation axioms diagram presented in FIGS 1 illustrates these implications.

We called the elements of the sequence $\left\langle\mathfrak{g}-\mathrm{T}_{\mathfrak{g}, \alpha}\right\rangle_{\alpha \in \Lambda} \mathfrak{g}-\mathrm{T}_{\mathfrak{g}, \alpha}$-axioms. To this end it does make sense to call those of $\left\langle\mathrm{T}_{\mathfrak{g}, \alpha}\right\rangle_{\alpha \in \Lambda} \mathrm{T}_{\mathfrak{g}, \alpha}$-axioms. Thus, in a $\mathcal{T}_{\mathfrak{g}}$-space $\mathfrak{T}_{\mathfrak{g}}=\left(\Omega, \mathcal{T}_{\mathfrak{g}}\right),\left\langle\mathrm{T}_{\mathfrak{g}, \alpha}\right\rangle_{\alpha \in \Lambda}$ stands for a sequence of separation axioms in the ordinary sense while $\left\langle\mathfrak{g}-T_{\mathfrak{g}, \alpha}\right\rangle_{\alpha \in \Lambda}$ stands for its analogue but in the generalized sense, just as, in a $\mathcal{T}$-space $\mathfrak{T}=(\Omega, \mathcal{T}),\left\langle\mathrm{T}_{\alpha}\right\rangle_{\alpha \in \Lambda}$ stands for a sequence of separation axioms in the ordinary sense while $\left\langle\mathfrak{g}-\mathrm{T}_{\alpha}\right\rangle_{\alpha \in \Lambda}$ stands for its analogue but in the generalized sense. Let $\mathrm{F}_{\mathrm{P}}[\sigma]=\mathfrak{g}-\mathrm{F}_{\mathrm{P}}[\sigma]$ and set

$$
\begin{aligned}
& \mathrm{F}_{\mathrm{O}}\left[\mathcal{S}_{\mathfrak{g}}\right] \stackrel{\text { def }}{=}\left\{\left(\mathcal{O}_{\mathfrak{g}, \xi}, \mathcal{O}_{\mathfrak{g}, \zeta}\right) \in \mathcal{T}_{\mathfrak{g}} \times \mathcal{T}_{\mathfrak{g}}: \bigcap_{\lambda=\xi, \zeta} \mathcal{O}_{\mathfrak{g}, \lambda} \subseteq \mathcal{S}_{\mathfrak{g}}\right\}, \\
& \mathrm{F}_{\mathrm{K}}\left[\mathcal{S}_{\mathfrak{g}}\right] \stackrel{\text { def }}{=}\left\{\left(\mathcal{K}_{\mathfrak{g}, \xi}, \mathcal{K}_{\mathfrak{g}, \zeta}\right) \in \neg \mathcal{T}_{\mathfrak{g}} \times \neg \mathcal{T}_{\mathfrak{g}}: \mathcal{S}_{\mathfrak{g}} \supseteq \bigcap_{\lambda=\xi, \zeta} \mathcal{K}_{\mathfrak{g}, \lambda}\right\},
\end{aligned}
$$

where $\sigma \geq 0$ and $\mathcal{S}_{\mathfrak{g}} \supseteq \emptyset$. Then, the notions of $\mathrm{T}_{\mathfrak{g}, \mathrm{K}}, \mathrm{T}_{\mathfrak{g}, \mathrm{F}}, \mathrm{T}_{\mathfrak{g}, \mathrm{H}}, \mathrm{T}_{\mathfrak{g}, \mathrm{R}}$, and $\mathrm{T}_{\mathfrak{g}, \mathrm{N}^{-}}$ axioms in a $\mathcal{T}_{\mathfrak{g}}$-space $\mathfrak{T}_{\mathfrak{g}}=\left(\Omega, \mathcal{T}_{\mathfrak{g}}\right)$ may well be defined as follows:

- I. $\mathrm{T}_{\mathfrak{g}, \mathrm{K}}$-AXIOM: For every $(\xi, \zeta) \in \mathrm{F}_{\mathrm{P}}[\sigma>0]$, there exists $\left(\mathcal{O}_{\mathfrak{g}, \xi}, \mathcal{O}_{\mathfrak{g}, \zeta}\right) \in$ $\mathrm{F}_{\mathrm{O}}\left[\mathcal{S}_{\mathfrak{g}} \supset \emptyset\right]$ such that:

$$
\left[\left(\xi \in \mathcal{O}_{\mathfrak{g}, \xi}\right) \wedge\left(\zeta \notin \mathcal{O}_{\mathfrak{g}, \xi}\right)\right] \vee\left[\left(\xi \notin \mathcal{O}_{\mathfrak{g}, \zeta}\right) \wedge\left(\zeta \in \mathcal{O}_{\mathfrak{g}, \zeta}\right)\right]
$$




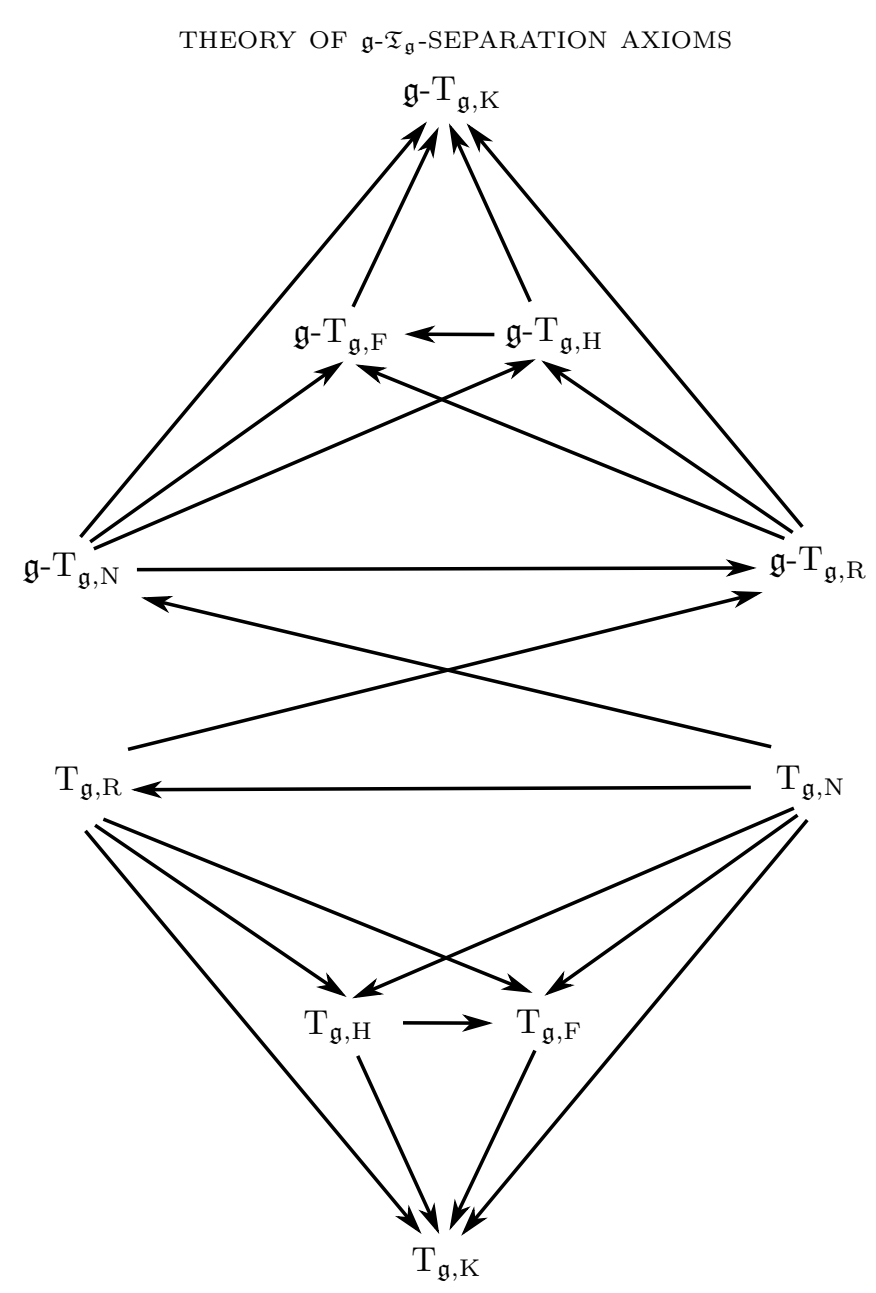

FiguRE 1. Relationships: Separation axioms diagram.

- II. $\mathrm{T}_{\mathfrak{g}, \mathrm{F}}$-AXIOM: For every $(\xi, \zeta) \in \mathrm{F}_{\mathrm{P}}[\sigma>0]$, there exists $\left(\mathcal{O}_{\mathfrak{g}, \xi}, \mathcal{O}_{\mathfrak{g}, \zeta}\right) \in$ $\mathrm{F}_{\mathrm{O}}\left[\mathcal{S}_{\mathfrak{g}} \supset \emptyset\right]$ such that:

$$
\left[(\xi, \zeta) \in \times_{\lambda=\xi, \zeta} \mathcal{O}_{\mathfrak{g}, \lambda}\right] \wedge\left[(\xi, \zeta) \notin \times_{\lambda=\zeta, \xi} \mathcal{O}_{\mathfrak{g}, \lambda}\right] .
$$

- III. $\mathrm{T}_{\mathfrak{g}, \mathrm{H}}$-Axiom: For every $(\xi, \zeta) \in \mathrm{F}_{\mathrm{P}}[\sigma>0]$, there exists $\left(\mathcal{O}_{\mathfrak{g}, \xi}, \mathcal{O}_{\mathfrak{g}, \zeta}\right) \in$ $\mathrm{F}_{\mathrm{O}}[\emptyset]$ such that:

$$
\left[\xi \in \mathcal{O}_{\mathfrak{g}, \xi}\right] \wedge\left[\zeta \in \mathcal{O}_{\mathfrak{g}, \zeta}\right]
$$

- IV. $\mathrm{T}_{\mathfrak{g}, \mathrm{R}}$-Axiom: For every $(\xi, \zeta) \in \mathrm{F}_{\mathrm{P}}[\sigma>0]$ and $\left(\mathcal{K}_{\mathfrak{g}, \xi}, \mathcal{K}_{\mathfrak{g}, \zeta}\right) \in \mathrm{F}_{\mathrm{K}}[\emptyset]$ such that $(\zeta, \xi) \notin\left(\mathcal{K}_{\mathfrak{g}, \xi}, \mathcal{K}_{\mathfrak{g}, \zeta}\right)$, there exists $\left(\mathcal{O}_{\mathfrak{g}, \xi}, \mathcal{O}_{\mathfrak{g}, \zeta}\right) \in \mathrm{F}_{\mathrm{O}}[\emptyset]$ such that:

$$
\left[\left(\mathcal{K}_{\mathfrak{g}, \xi} \subset \mathcal{O}_{\mathfrak{g}, \xi}\right) \wedge\left(\zeta \in \mathcal{O}_{\mathfrak{g}, \zeta}\right)\right] \vee\left[\left(\mathcal{K}_{\mathfrak{g}, \zeta} \subset \mathcal{O}_{\mathfrak{g}, \zeta}\right) \wedge\left(\xi \in \mathcal{O}_{\mathfrak{g}, \xi}\right)\right] .
$$

- v. $\mathrm{T}_{\mathfrak{g}, \mathrm{N}}$-AxiOM: For every $\left(\mathcal{K}_{\mathfrak{g}, \xi}, \mathcal{K}_{\mathfrak{g}, \zeta}\right) \in \mathrm{F}_{\mathrm{K}}[\emptyset]$, there exists $\left(\mathcal{O}_{\mathfrak{g}, \xi}, \mathcal{O}_{\mathfrak{g}, \zeta}\right) \in$ $\mathrm{F}_{\mathrm{O}}[\emptyset]$ such that:

$$
\left[\mathcal{O}_{\mathfrak{g}, \xi} \supset \mathcal{K}_{\mathfrak{g}, \xi}\right] \wedge\left[\mathcal{O}_{\mathfrak{g}, \zeta} \supset \mathcal{K}_{\mathfrak{g}, \zeta}\right]
$$


By virtue of the relations $\mathcal{O}_{\mathfrak{g}, \lambda} \subseteq \operatorname{op}_{\mathfrak{g}}\left(\mathcal{O}_{\mathfrak{g}, \lambda}\right)$ and $\mathcal{K}_{\mathfrak{g}, \lambda} \supseteq \neg \mathrm{op}_{\mathfrak{g}}\left(\mathcal{K}_{\mathfrak{g}, \lambda}\right)$ for every $\left(\mathcal{O}_{\mathfrak{g}, \lambda}, \mathcal{K}_{\mathfrak{g}, \lambda}, \lambda\right) \in \mathcal{T}_{\mathfrak{g}} \times \neg \mathcal{T}_{\mathfrak{g}} \times\{\xi, \zeta\}$, these implications follow: $\mathfrak{g}-\mathrm{T}_{\mathfrak{g}, \mathrm{K}} \leftarrow \mathrm{T}_{\mathfrak{g}, \mathrm{K}}$, $\mathfrak{g}-T_{\mathfrak{g}, \mathrm{F}} \leftarrow \mathrm{T}_{\mathfrak{g}, \mathrm{F}}, \mathfrak{g}-\mathrm{T}_{\mathfrak{g}, \mathrm{H}} \leftarrow \mathrm{T}_{\mathfrak{g}, \mathrm{H}}, \mathfrak{g}-\mathrm{T}_{\mathfrak{g}, \mathrm{R}} \leftarrow \mathrm{T}_{\mathfrak{g}, \mathrm{R}}$, and $\mathfrak{g}-\mathrm{T}_{\mathfrak{g}, \mathrm{N}} \leftarrow \mathrm{T}_{\mathfrak{g}, \mathrm{N}}$. When the statements preceding the above definitions are taken into account, another separation axioms diagram is obtained. In FIG. 2, we have illustrated the various relationships amongst the elements of $\left\langle\mathfrak{g}-\mathrm{T}_{\mathfrak{g}, \alpha}\right\rangle_{\alpha \in \Lambda}$ and $\left\langle\mathrm{T}_{\mathfrak{g}, \alpha}\right\rangle_{\alpha \in \Lambda}$. It is interesting to present a third separation axioms diagram illustrating both the implications and the categorical classifications of the elements of $\left\langle\mathfrak{g}-\nu-\mathrm{T}_{\mathfrak{g}, \alpha}\right\rangle_{\alpha \in \Lambda}$, where $\nu \in I_{3}^{0}$.

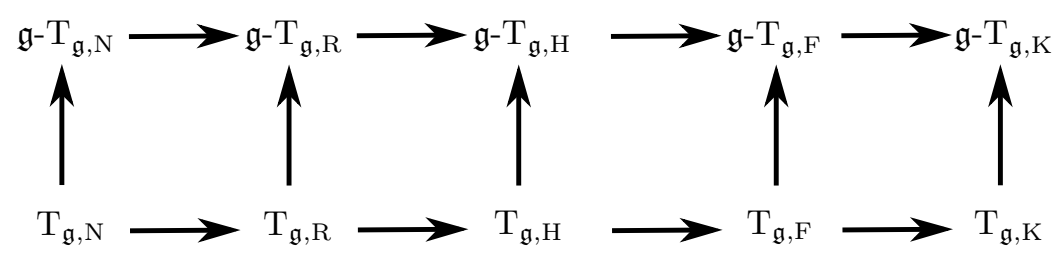

Figure 2. Relationships: Separation axioms diagram.

For every fixed $\nu \in I_{3}^{0}$, it is immediate that the implications $\mathfrak{g}-\nu$ - $\mathrm{T}_{\mathfrak{g}, \mathrm{K}} \leftarrow$ $\mathfrak{g}-\nu-\mathrm{T}_{\mathfrak{g}, \mathrm{F}}, \mathfrak{g}-\nu-\mathrm{T}_{\mathfrak{g}, \mathrm{F}} \leftarrow \mathfrak{g}-\nu-\mathrm{T}_{\mathfrak{g}, \mathrm{H}}, \mathfrak{g}-\nu-\mathrm{T}_{\mathfrak{g}, \mathrm{H}} \leftarrow \mathfrak{g}-\nu-\mathrm{T}_{\mathfrak{g}, \mathrm{R}}$, and $\mathfrak{g}-\nu-\mathrm{T}_{\mathfrak{g}, \mathrm{R}} \leftarrow \mathfrak{g}-\nu-\mathrm{T}_{\mathfrak{g}, \mathrm{N}}$ hold. On the other hand, we saw in the first part of our works, on the theory of $\mathfrak{g}-\mathfrak{T}_{\mathfrak{g}}$-sets, that

$$
\begin{aligned}
\operatorname{op}_{\mathfrak{g}, 0}\left(\mathcal{S}_{\mathfrak{g}}\right) \subseteq \mathrm{op}_{\mathfrak{g}, 1}\left(\mathcal{S}_{\mathfrak{g}}\right) \subseteq \mathrm{op}_{\mathfrak{g}, 3}\left(\mathcal{S}_{\mathfrak{g}}\right) \supseteq \mathrm{op}_{\mathfrak{g}, 2}\left(\mathcal{S}_{\mathfrak{g}}\right) \forall \mathcal{S}_{\mathfrak{g}} \subset \mathfrak{T}_{\mathfrak{g}}, \\
\neg \mathrm{op}_{\mathfrak{g}, 0}\left(\mathcal{S}_{\mathfrak{g}}\right) \supseteq \neg \mathrm{op}_{\mathfrak{g}, 1}\left(\mathcal{S}_{\mathfrak{g}}\right) \supseteq \neg \mathrm{op}_{\mathfrak{g}, 3}\left(\mathcal{S}_{\mathfrak{g}}\right) \subseteq \neg \mathrm{op}_{\mathfrak{g}, 2}\left(\mathcal{S}_{\mathfrak{g}}\right),
\end{aligned}
$$

as a consequence of our definitions of the $\mathfrak{g}$-operators op $\mathfrak{g}_{\mathfrak{g}, \nu}, \neg \mathrm{op}_{\mathfrak{g}, \nu}: \mathcal{P}(\Omega) \rightarrow \mathcal{P}(\Omega)$. Hence, for every $\alpha \in \Lambda, \mathfrak{g}-0-\mathrm{T}_{\mathfrak{g}, \alpha} \rightarrow \mathfrak{g}-1-\mathrm{T}_{\mathfrak{g}, \alpha} \rightarrow \mathfrak{g}-3-\mathrm{T}_{\mathfrak{g}, \alpha}$ and $\mathfrak{g}-3-\mathrm{T}_{\mathfrak{g}, \alpha} \leftarrow \mathfrak{g}-2-\mathrm{T}_{\mathfrak{g}, \alpha}$. When these properties are taken into consideration, the resulting separation axioms diagram so obtained is that presented in FIG. 3. It is reasonable to call them $\mathfrak{g}-\mathrm{T}_{\mathfrak{g}, \alpha}$-axioms of type $\alpha$ and of category $\nu$, where $(\alpha, \nu) \in \Lambda \times I_{3}^{0}$.

In order to exemplify the concept of $\mathfrak{g}-\mathrm{T}_{\mathfrak{g}, \alpha}$-axiom of type $\alpha$ and of category $\nu$, where $(\alpha, \nu) \in \Lambda \times I_{3}^{0}$, a nice application is presented in the following section.

4.2. A Nice Applichtion. Focusing on the fundamental notions of the sequence $\left\langle\mathfrak{g}-\mathrm{T}_{\mathfrak{g}, \alpha}\right\rangle_{\alpha \in \Lambda}$ of $\mathfrak{g}$-T $\mathrm{T}_{\mathfrak{g}, \alpha}$-axioms, $\Lambda=\{\mathrm{K}, \mathrm{F}, \mathrm{H}, \mathrm{R}, \mathrm{N}\}$, in a $\mathcal{T}_{\mathfrak{g}}$-space, founded upon the class of $\mathfrak{g}-\mathcal{T}_{\mathfrak{g}}$-open sets, we shall now present a nice application. Let $\Omega=\left\{\xi_{\nu}\right.$ : $\left.\nu \in I_{3}^{*}\right\}$ denotes the underlying set and consider the $\mathcal{T}_{\mathfrak{g}}$-space $\mathfrak{T}_{\mathfrak{g}}=\left(\Omega, \mathcal{T}_{\mathfrak{g}}\right)$, where

$$
\begin{aligned}
\mathcal{T}_{\mathfrak{g}}(\Omega) & =\left\{\emptyset,\left\{\xi_{1}\right\},\left\{\xi_{2}\right\},\left\{\xi_{3}\right\},\left\{\xi_{1}, \xi_{2}\right\},\left\{\xi_{1}, \xi_{3}\right\},\left\{\xi_{2}, \xi_{3}\right\}, \Omega\right\} \\
& =\left\{\mathcal{O}_{\mathfrak{g}, 1}, \mathcal{O}_{\mathfrak{g}, 2}, \mathcal{O}_{\mathfrak{g}, 3}, \mathcal{O}_{\mathfrak{g}, 4}, \mathcal{O}_{\mathfrak{g}, 5}, \mathcal{O}_{\mathfrak{g}, 6}, \mathcal{O}_{\mathfrak{g}, 7}, \mathcal{O}_{\mathfrak{g}, 8}\right\}, \\
\neg \mathcal{T}_{\mathfrak{g}}(\Omega) & =\left\{\Omega,\left\{\xi_{2}, \xi_{3}\right\},\left\{\xi_{1}, \xi_{3}\right\},\left\{\xi_{1}, \xi_{2}\right\},\left\{\xi_{3}\right\},\left\{\xi_{2}\right\},\left\{\xi_{1}\right\}, \emptyset\right\} \\
& =\left\{\mathcal{K}_{\mathfrak{g}, 1}, \mathcal{K}_{\mathfrak{g}, 2}, \mathcal{K}_{\mathfrak{g}, 3}, \mathcal{K}_{\mathfrak{g}, 4}, \mathcal{K}_{\mathfrak{g}, 5}, \mathcal{K}_{\mathfrak{g}, 6}, \mathcal{K}_{\mathfrak{g}, 7}, \mathcal{K}_{\mathfrak{g}, 8}\right\},
\end{aligned}
$$

respectively, stand for the classes of $\mathcal{T}_{\mathfrak{g}}$-open and $\mathcal{T}_{\mathfrak{g}}$-closed sets. In both settings, the $\mathcal{T}_{\mathfrak{g}}$-open, closed sets occupying the $\nu^{\text {th }}$ position corresponds to $\mathcal{O}_{\mathfrak{g}, \nu}, \mathcal{K}_{\mathfrak{g}, \nu}, \nu \in I_{8}^{*}$, respectively, as is easily understood. Since conditions $\mathcal{T}_{\mathfrak{g}}(\emptyset)=\emptyset, \mathcal{T}_{\mathfrak{g}}\left(\mathcal{O}_{\mathfrak{g}, \nu}\right) \subseteq \mathcal{O}_{\mathfrak{g}, \nu}$ for every $\nu \in I_{8}^{*}$, and $\mathcal{T}_{\mathfrak{g}}\left(\bigcup_{\nu \in I_{8}^{*}} \mathcal{O}_{\mathfrak{g}, \nu}\right)=\bigcup_{\nu \in I_{8}^{*}} \mathcal{T}_{\mathfrak{g}}\left(\mathcal{O}_{\mathfrak{g}, \nu}\right)$ are satisfied, it is clear that the one-valued map $\mathcal{T}_{\mathfrak{g}}: \mathcal{P}(\Omega) \rightarrow \mathcal{P}\left(\left\{\xi_{\nu}: \nu \in I_{8}^{*}\right\}\right)$ is a $\mathfrak{g}$-topology. After 
THEORY OF $\mathfrak{g}$ - $\mathfrak{T}_{\mathfrak{g}}$-SEPARATION AXIOMS

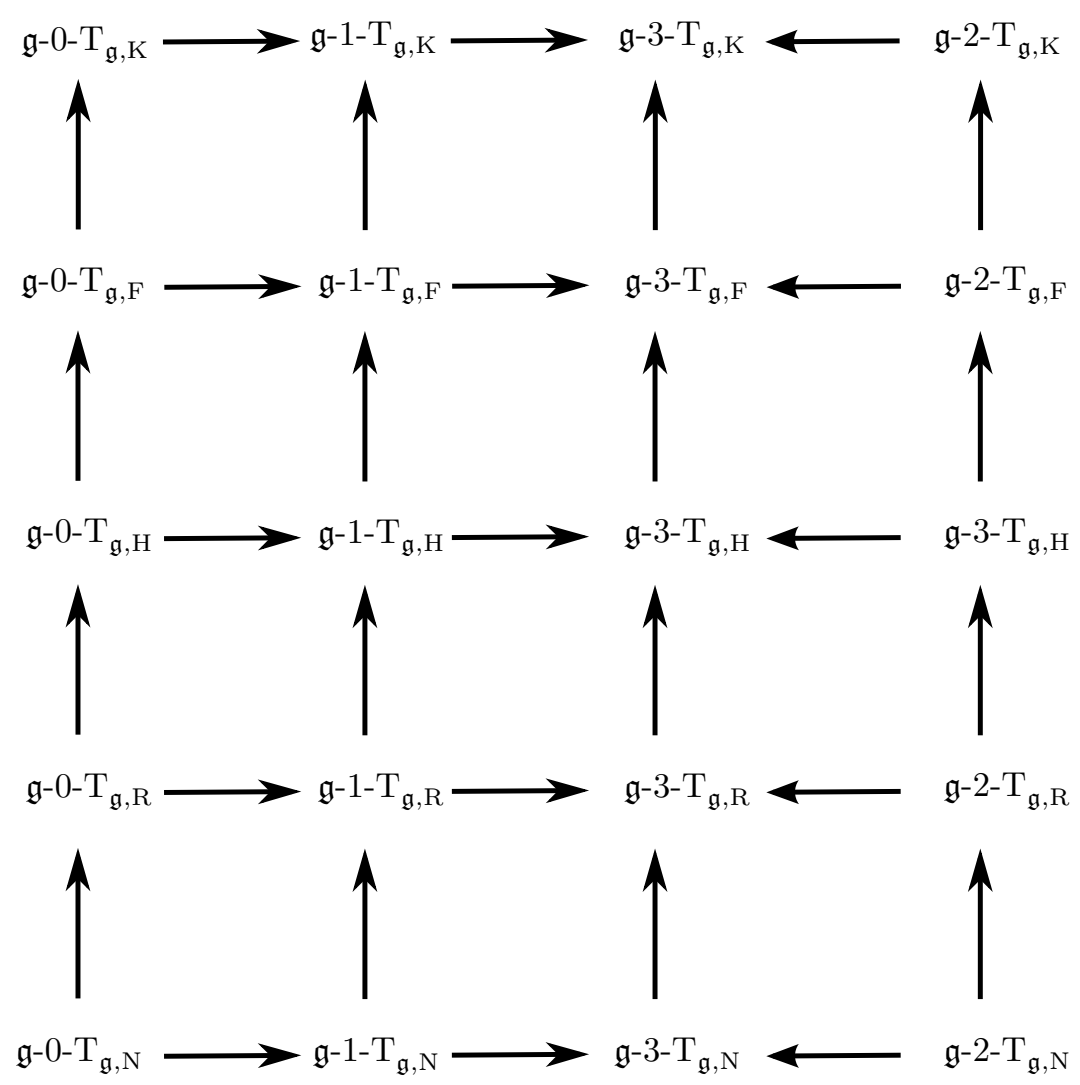

FiguRE 3. Relationships: Separation axiom diagram.

computing the elements of the set $\left\{\operatorname{op}_{\mathfrak{g}, \nu}\left(\mathcal{O}_{\mathfrak{g}, \xi_{\alpha}}\right):(\alpha, \nu) \in I_{8}^{*} \times I_{3}^{0}\right\}$, called $\mathfrak{g}-\mathcal{T}_{\mathfrak{g}^{-}}$ open sets, we obtain:

$$
\operatorname{op}_{\mathfrak{g}, \nu}\left(\mathcal{O}_{\mathfrak{g}, \xi_{\alpha}}\right) \in \begin{cases}\left\{\mathcal{O}_{\mathfrak{g}, 2}, \mathcal{O}_{\mathfrak{g}, 5}, \mathcal{O}_{\mathfrak{g}, 6}, \mathcal{O}_{\mathfrak{g}, 8}\right\} & \forall(\alpha, \nu) \in\{1\} \times\{0,2\}, \\ \left\{\mathcal{O}_{\mathfrak{g}, 3}, \mathcal{O}_{\mathfrak{g}, 5}, \mathcal{O}_{\mathfrak{g}, 7}, \mathcal{O}_{\mathfrak{g}, 8}\right\} & \forall(\alpha, \nu) \in\{2\} \times\{0,2\}, \\ \left\{\mathcal{O}_{\mathfrak{g}, 4}, \mathcal{O}_{\mathfrak{g}, 6}, \mathcal{O}_{\mathfrak{g}, 7}, \mathcal{O}_{\mathfrak{g}, 8}\right\} & \forall(\alpha, \nu) \in\{3\} \times\{0,2\}, \\ \left\{\mathcal{K}_{\mathfrak{g}, 1}, \mathcal{K}_{\mathfrak{g}, 3}, \mathcal{K}_{\mathfrak{g}, 4}, \mathcal{K}_{\mathfrak{g}, 7}\right\} & \forall(\alpha, \nu) \in\{1\} \times\{1,3\}, \\ \left\{\mathcal{K}_{\mathfrak{g}, 1}, \mathcal{K}_{\mathfrak{g}, 2}, \mathcal{K}_{\mathfrak{g}, 4}, \mathcal{K}_{\mathfrak{g}, 6}\right\} & \forall(\alpha, \nu) \in\{2\} \times\{1,3\}, \\ \left\{\mathcal{K}_{\mathfrak{g}, 1}, \mathcal{K}_{\mathfrak{g}, 2}, \mathcal{K}_{\mathfrak{g}, 3}, \mathcal{K}_{\mathfrak{g}, 5}\right\} & \forall(\alpha, \nu) \in\{3\} \times\{1,3\} .\end{cases}
$$


Similarly, the elements of $\left\{\neg \mathrm{op}_{\mathfrak{g}, \nu}\left(\mathcal{K}_{\mathfrak{g}, \xi_{\alpha}}\right):(\alpha, \nu) \in I_{8}^{*} \times I_{3}^{0}\right\}$, called $\mathfrak{g}$ - $\mathcal{T}_{\mathfrak{g}}$-closed sets, are:

$$
\neg \mathrm{op}_{\mathfrak{g}, \nu}\left(\mathcal{K}_{\mathfrak{g}, \xi_{\alpha}}\right) \in \begin{cases}\left\{\mathcal{K}_{\mathfrak{g}, 1}, \mathcal{K}_{\mathfrak{g}, 3}, \mathcal{K}_{\mathfrak{g}, 4}, \mathcal{K}_{\mathfrak{g}, 7}\right\} & \forall(\alpha, \nu) \in\{1\} \times\{0,2\}, \\ \left\{\mathcal{K}_{\mathfrak{g}, 1}, \mathcal{K}_{\mathfrak{g}, 2}, \mathcal{K}_{\mathfrak{g}, 4}, \mathcal{K}_{\mathfrak{g}, 6}\right\} & \forall(\alpha, \nu) \in\{2\} \times\{0,2\}, \\ \left\{\mathcal{K}_{\mathfrak{g}, 1}, \mathcal{K}_{\mathfrak{g}, 2}, \mathcal{K}_{\mathfrak{g}, 3}, \mathcal{K}_{\mathfrak{g}, 5}\right\} & \forall(\alpha, \nu) \in\{3\} \times\{0,2\}, \\ \left\{\mathcal{O}_{\mathfrak{g}, 2}, \mathcal{O}_{\mathfrak{g}, 5}, \mathcal{O}_{\mathfrak{g}, 6}, \mathcal{O}_{\mathfrak{g}, 8}\right\} & \forall(\alpha, \nu) \in\{1\} \times\{1,3\}, \\ \left\{\mathcal{O}_{\mathfrak{g}, 3}, \mathcal{O}_{\mathfrak{g}, 5}, \mathcal{O}_{\mathfrak{g}, 7}, \mathcal{O}_{\mathfrak{g}, 8}\right\} & \forall(\alpha, \nu) \in\{2\} \times\{1,3\}, \\ \left\{\mathcal{O}_{\mathfrak{g}, 4}, \mathcal{O}_{\mathfrak{g}, 6}, \mathcal{O}_{\mathfrak{g}, 7}, \mathcal{O}_{\mathfrak{g}, 8}\right\} & \forall(\alpha, \nu) \in\{3\} \times\{1,3\} .\end{cases}
$$

First, for every $\nu \in I_{3}^{0}$, set $\mathcal{I}_{\mathfrak{g},\left(\xi_{\alpha}, \xi_{\beta}\right)}^{\mathrm{op}}=\bigcap_{\lambda=\alpha, \beta} \mathrm{op}_{\mathfrak{g}, \nu}\left(\mathcal{O}_{\mathfrak{g}, \xi_{\lambda}}\right)$ and $\mathcal{I}_{\mathfrak{g},\left(\xi_{\alpha}, \xi_{\beta}\right)}^{\mathrm{cl}}=$ $\bigcap_{\lambda=\alpha, \beta} \neg \mathrm{op}_{\mathfrak{g}, \nu}\left(\mathcal{K}_{\mathfrak{g}, \xi_{\lambda}}\right)$. Next, for all $(\alpha, \beta, \nu) \in I_{3}^{*} \times I_{3}^{*} \times I_{3}^{0}$, calculate $\mathcal{I}_{\mathfrak{g},\left(\xi_{\alpha}, \xi_{\beta}\right)}^{\text {op }}$, $\mathcal{I}_{\mathfrak{g},\left(\xi_{\alpha}, \xi_{\beta}\right)}^{\mathrm{cl}}$. Finally, for every $(r, s) \in I_{8}^{*} \times I_{8}^{*}$, set $\mathcal{O}_{\mathfrak{g},(r, s)}=\left(\mathcal{O}_{\mathfrak{g}, r}, \mathcal{O}_{\mathfrak{g}, s}\right)$ and $\mathcal{K}_{\mathfrak{g},(r, s)}=\left(\mathcal{K}_{\mathfrak{g}, r}, \mathcal{K}_{\mathfrak{g}, s}\right)$. These procedures yield:

$$
\begin{aligned}
\mathfrak{g}-\mathrm{F}_{\mathrm{P}}[\sigma>0]= & \bigcup_{\alpha \in I_{3}^{*}}\left\{\left(\xi_{\alpha}, \xi_{\beta}\right): \beta \in I_{3}^{*} \backslash\{\alpha\}\right\}, \\
\mathfrak{g}-\nu-\mathrm{F}_{\mathrm{O}}[\emptyset]= & \left\{\mathcal{O}_{\mathfrak{g},(3,2)}, \mathcal{O}_{\mathfrak{g},(3,6)}, \mathcal{O}_{\mathfrak{g},(4,2)}, \mathcal{O}_{\mathfrak{g},(4,3)},\right. \\
& \left.\mathcal{O}_{\mathfrak{g},(4,5)}, \mathcal{O}_{\mathfrak{g},(6,3)}, \mathcal{O}_{\mathfrak{g},(7,2)}\right\}, \\
\mathfrak{g}-\nu-\mathrm{F}_{\mathrm{K}}[\emptyset]= & \left\{\mathcal{K}_{\mathfrak{g},(2,7)}, \mathcal{K}_{\mathfrak{g},(3,6)}, \mathcal{K}_{\mathfrak{g},(5,4)}, \mathcal{K}_{\mathfrak{g},(5,6),}\right. \\
& \left.\mathcal{K}_{\mathfrak{g},(5,7)}, \mathcal{K}_{\mathfrak{g},(6,3)}, \mathcal{K}_{\mathfrak{g},(6,7)}\right\}, \\
\mathfrak{g}-\nu-\mathrm{F}_{\mathrm{O}}\left[\mathcal{S}_{\mathfrak{g}} \supset \emptyset\right]= & \left\{\mathcal{O}_{\mathfrak{g},(r, s)}:(r, s) \in I_{8}^{*} \times I_{8}^{*}\right\} \supset \mathfrak{g}-\nu-\mathrm{F}_{\mathrm{O}}[\emptyset] \quad \forall \nu \in I_{3}^{0}, \\
\mathfrak{g}-\nu-\mathrm{F}_{\mathrm{K}}\left[\mathcal{S}_{\mathfrak{g}} \supset \emptyset\right]= & \left\{\mathcal{K}_{\mathfrak{g},(r, s)}:(r, s) \in I_{8}^{*} \times I_{8}^{*}\right\} \supset \mathfrak{g}-\nu-\mathrm{F}_{\mathrm{K}}[\emptyset] .
\end{aligned}
$$

We are now in a position to discuss the $\mathfrak{g}-\mathrm{T}_{\mathfrak{g}, \alpha}$-axioms, $\Lambda=\{\mathrm{K}, \mathrm{F}, \mathrm{H}, \mathrm{R}, \mathrm{N}\}$.

Let $\mathcal{O}_{\mathfrak{g},(p, q)} \supset \mathcal{K}_{\mathfrak{g},(r, s)}$ mean $\mathcal{O}_{\mathfrak{g}, p} \supset \mathcal{K}_{\mathfrak{g}, r}$ and $\mathcal{O}_{\mathfrak{g}, q} \supset \mathcal{K}_{\mathfrak{g}, s}$, where $\mathcal{O}_{\mathfrak{g},(p, q)} \in$ $\mathfrak{g}-\nu-\mathrm{F}_{\mathrm{O}}[\emptyset]$ and $\mathcal{K}_{\mathfrak{g},(r, s)} \in \mathfrak{g}-\nu-\mathrm{F}_{\mathrm{K}}[\emptyset]$. Further, for every $\nu \in I_{3}^{0}$, let op $\mathfrak{g}_{\mathfrak{g}, \nu}\left(\mathcal{O}_{\mathfrak{g},(p, q)}\right) \supset$ $\neg \mathrm{op}_{\mathfrak{g}, \nu}\left(\mathcal{K}_{\mathfrak{g},(r, s)}\right)$ mean $\operatorname{op}_{\mathfrak{g}, \nu}\left(\mathcal{O}_{\mathfrak{g}, p}\right) \supset \neg \mathrm{op}_{\mathfrak{g}, \nu}\left(\mathcal{K}_{\mathfrak{g}, r}\right)$, op $\mathfrak{g}_{\mathfrak{g}, \nu}\left(\mathcal{O}_{\mathfrak{g}, q}\right) \supset \neg \operatorname{op}_{\mathfrak{g}, \nu}\left(\mathcal{K}_{\mathfrak{g}, s}\right)$. Then, the following relations are easily checked: $\mathcal{O}_{\mathfrak{g},(7,2)} \supset \mathcal{K}_{\mathfrak{g},(2,7)} ; \mathcal{O}_{\mathfrak{g},(6,3)} \supset$ $\mathcal{K}_{\mathfrak{g},(3,6)} ; \mathcal{O}_{\mathfrak{g},(4,5)} \supset \mathcal{K}_{\mathfrak{g},(5,4)} ; \mathcal{O}_{\mathfrak{g},(4,3)} \supset \mathcal{K}_{\mathfrak{g},(5,6)} ; \mathcal{O}_{\mathfrak{g},(4,2)} \supset \mathcal{K}_{\mathfrak{g},(5,7)} ; \mathcal{O}_{\mathfrak{g},(3,6)} \supset \mathcal{K}_{\mathfrak{g},(6,3)}$ and $\mathcal{O}_{\mathfrak{g},(3,2)} \supset \mathcal{K}_{\mathfrak{g},(6,7)}$. But, for every $\nu \in I_{3}^{0}$, the relations $\mathcal{O}_{\mathfrak{g},(p, q)} \subseteq \operatorname{op}_{\mathfrak{g}, \nu}\left(\mathcal{O}_{\mathfrak{g},(p, q)}\right)$ and $\mathcal{K}_{\mathfrak{g},(r, s)} \supseteq \neg \mathrm{op}_{\mathfrak{g}, \nu}\left(\mathcal{K}_{\mathfrak{g},(r, s)}\right)$ hold for all $(p, q)=(3,2),(3,6),(4,2),(4,3),(4,5)$, $(6,3),(7,2)$ and all $(r, s)=(6,7),(6,3),(5,7),(5,6),(5,4),(3,6),(2,7)$. Combining these last two relations with $\mathcal{O}_{\mathfrak{g},(p, q)} \supset \mathcal{K}_{\mathfrak{g},(r, s)}$, it follows that op $\operatorname{gp}_{\mathfrak{g}, \nu}\left(\mathcal{O}_{\mathfrak{g},(p, q)}\right) \supset$ $\neg \mathrm{op}_{\mathfrak{g}, \nu}\left(\mathcal{K}_{\mathfrak{g},(r, s)}\right)$. Hence, for every $\mathcal{K}_{\mathfrak{g},(r, s)} \in \mathfrak{g}-\nu-\mathrm{F}_{\mathrm{K}}[\emptyset]$, there exists $\mathcal{O}_{\mathfrak{g},(p, q)} \in$ $\mathfrak{g}-\nu-\mathrm{F}_{\mathrm{O}}[\emptyset]$ such that:

$$
\left[\operatorname{op}_{\mathfrak{g}, \nu}\left(\mathcal{O}_{\mathfrak{g}, p}\right) \supset \neg \mathrm{op}_{\mathfrak{g}, \nu}\left(\mathcal{K}_{\mathfrak{g}, r}\right)\right] \wedge\left[\mathrm{op}_{\mathfrak{g}, \nu}\left(\mathcal{O}_{\mathfrak{g}, q}\right) \supset \neg \mathrm{op}_{\mathfrak{g}, \nu}\left(\mathcal{K}_{\mathfrak{g}, s}\right)\right] .
$$

This shows that $\mathfrak{T}_{\mathfrak{g}}$ is a $\mathfrak{g}-\mathcal{T}_{\mathfrak{g}}^{(\mathrm{N})}$-space $\mathfrak{g}-\mathfrak{T}_{\mathfrak{g}}^{(\mathrm{N})}=\left(\Omega, \mathfrak{g}-\mathcal{T}_{\mathfrak{g}}^{(\mathrm{N})}\right)$.

Let $\left(\xi_{i}, \xi_{j}\right) \in \mathcal{K}_{\mathfrak{g},(r, s)}$ mean $\xi_{i} \in \mathcal{K}_{\mathfrak{g}, r}$ and $\xi_{j} \in \mathcal{K}_{\mathfrak{g}, s}$, where $\mathcal{K}_{\mathfrak{g},(r, s)} \in \mathfrak{g}-\nu-\mathrm{F}_{\mathrm{K}}[\emptyset]$. Then, the following results are easily checked: $\left(\xi_{2}, \xi_{1}\right) \in \mathcal{K}_{\mathfrak{g},(2,7)}, \mathcal{K}_{\mathfrak{g},(6,3)}, \mathcal{K}_{\mathfrak{g},(6,7)}$ 
and $\left(\xi_{1}, \xi_{2}\right) \notin \mathcal{K}_{\mathfrak{g},(2,7)}, \mathcal{K}_{\mathfrak{g},(6,3)}, \mathcal{K}_{\mathfrak{g},(6,7)} ;\left(\xi_{3}, \xi_{1}\right) \in \mathcal{K}_{\mathfrak{g},(2,7)}, \mathcal{K}_{\mathfrak{g},(5,4)}, \mathcal{K}_{\mathfrak{g},(5,7)}$ and $\left(\xi_{1}, \xi_{3}\right) \notin \mathcal{K}_{\mathfrak{g},(2,7)}, \mathcal{K}_{\mathfrak{g},(5,4)}, \mathcal{K}_{\mathfrak{g},(5,7)} ;\left(\xi_{3}, \xi_{2}\right) \in \mathcal{K}_{\mathfrak{g},(3,6)}, \mathcal{K}_{\mathfrak{g},(5,4)}, \mathcal{K}_{\mathfrak{g},(5,6)}$ and $\left(\xi_{2}, \xi_{3}\right) \notin$ $\mathcal{K}_{\mathfrak{g},(3,6)}, \mathcal{K}_{\mathfrak{g},(5,4)}, \mathcal{K}_{\mathfrak{g},(5,6)}$. But, $\mathcal{O}_{\mathfrak{g},(7,2)} \supset \mathcal{K}_{\mathfrak{g},(2,7)} ; \mathcal{O}_{\mathfrak{g},(6,3)} \supset \mathcal{K}_{\mathfrak{g},(3,6)} ; \mathcal{O}_{\mathfrak{g},(4,5)} \supset$ $\mathcal{K}_{\mathfrak{g},(5,4)} ; \mathcal{O}_{\mathfrak{g},(4,3)} \supset \mathcal{K}_{\mathfrak{g},(5,6)} ; \mathcal{O}_{\mathfrak{g},(4,2)} \supset \mathcal{K}_{\mathfrak{g},(5,7)} ; \mathcal{O}_{\mathfrak{g},(3,6)} \supset \mathcal{K}_{\mathfrak{g},(6,3)}$ and $\mathcal{O}_{\mathfrak{g},(3,2)} \supset$

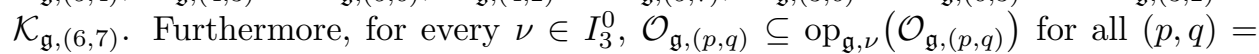
$(3,2),(3,6),(4,2),(4,3),(4,5),(6,3),(7,2)$ and $\mathcal{K}_{\mathfrak{g},(r, s)} \supseteq \neg \mathrm{op}_{\mathfrak{g}, \nu}\left(\mathcal{K}_{\mathfrak{g},(r, s)}\right)$ for all $(r, s)=(2,7),(3,6),(5,4),(5,6),(5,7),(6,3),(6,7)$. Thus, for every $\left(\xi_{i}, \xi_{j}\right) \in$ $\mathfrak{g}-\mathrm{F}_{\mathrm{P}}[\sigma>0]$ and $\mathcal{K}_{\mathfrak{g},(r, s)} \in \mathfrak{g}-\nu-\mathrm{F}_{\mathrm{K}}[\emptyset]$ such that $\left(\xi_{j}, \xi_{i}\right) \notin \mathcal{K}_{\mathfrak{g},(r, s)}$, there exists $\mathcal{O}_{\mathfrak{g},(p, q)} \in \mathfrak{g}-\nu-\mathrm{F}_{\mathrm{O}}[\emptyset]$ such that:

$$
\begin{aligned}
{\left[\left(\neg \mathrm{op}_{\mathfrak{g}, \nu}\left(\mathcal{K}_{\mathfrak{g}, r}\right)\right.\right.} & \left.\subset \mathrm{op}_{\mathfrak{g}, \nu}\left(\mathcal{O}_{\mathfrak{g}, p}\right)\right) \\
\left.\subset \mathrm{op}_{\mathfrak{g}, \nu}\left(\mathcal{O}_{\mathfrak{g}, q}\right)\right) & \left.\wedge\left(\zeta \in \mathrm{op}_{\mathfrak{g}, \nu}\left(\mathcal{O}_{\mathfrak{g}, q}\right)\right)\right] \vee\left[\left(\neg \mathrm{op}_{\mathfrak{g}, \nu}\left(\mathcal{O}_{\mathfrak{g}, p}\right)\right)\right] .
\end{aligned}
$$

This shows that $\mathfrak{T}_{\mathfrak{g}}$ is a $\mathfrak{g}-\mathcal{T}_{\mathfrak{g}}^{(\mathrm{R})}$-space $\mathfrak{g}-\mathfrak{T}_{\mathfrak{g}}^{(\mathrm{R})}=\left(\Omega, \mathfrak{g}-\mathcal{T}_{\mathfrak{g}}^{(\mathrm{R})}\right)$.

Let $\left(\xi_{i}, \xi_{j}\right) \in \mathcal{O}_{\mathfrak{g},(p, q)}$ mean $\xi_{i} \in \mathcal{O}_{\mathfrak{g}, p}$ and $\xi_{j} \in \mathcal{O}_{\mathfrak{g}, q}$, where $\mathcal{O}_{\mathfrak{g},(p, q)} \in \mathfrak{g}-\nu-\mathrm{F}_{\mathrm{O}}[\emptyset]$. Then, the following relations are easily verified: $\left(\xi_{2}, \xi_{1}\right) \in \mathcal{O}_{\mathfrak{g},(3,2)}, \mathcal{O}_{\mathfrak{g},(3,6)}, \mathcal{O}_{\mathfrak{g},(7,2)}$ and $\left(\xi_{1}, \xi_{2}\right) \notin \mathcal{O}_{\mathfrak{g},(3,2)}, \mathcal{O}_{\mathfrak{g},(3,6)}, \mathcal{O}_{\mathfrak{g},(7,2)} ;\left(\xi_{3}, \xi_{1}\right) \in \mathcal{O}_{\mathfrak{g},(4,2)}, \mathcal{O}_{\mathfrak{g},(4,5)}, \mathcal{O}_{\mathfrak{g},(7,2)}$ and $\left(\xi_{1}, \xi_{3}\right) \notin \mathcal{O}_{\mathfrak{g},(4,2)}, \mathcal{O}_{\mathfrak{g},(4,5)}, \mathcal{O}_{\mathfrak{g},(7,2)} ;\left(\xi_{3}, \xi_{2}\right) \in \mathcal{O}_{\mathfrak{g},(4,3)}, \mathcal{O}_{\mathfrak{g},(4,5)}, \mathcal{O}_{\mathfrak{g},(6,3)}$ and $\left(\xi_{2}, \xi_{3}\right) \notin$ $\mathcal{O}_{\mathfrak{g},(4,3)}, \mathcal{O}_{\mathfrak{g},(4,5)}, \mathcal{O}_{\mathfrak{g},(6,3)}$. But, for every $\nu \in I_{3}^{0}, \mathcal{O}_{\mathfrak{g},(p, q)} \subseteq \mathrm{op}_{\mathfrak{g}, \nu}\left(\mathcal{O}_{\mathfrak{g},(p, q)}\right)$ and $\mathcal{O}_{\mathfrak{g},(p, q)} \in \mathfrak{g}-\nu-\mathrm{F}_{\mathrm{O}}[\emptyset]$ for all $(p, q)=(3,2),(3,6),(4,2),(4,3),(4,5),(6,3),(7,2)$. Thus, for every $\left(\xi_{i}, \xi_{j}\right) \in \mathfrak{g}-\mathrm{F}_{\mathrm{P}}[\sigma>0]$, there exists $\mathcal{O}_{\mathfrak{g},(p, q)} \in \mathfrak{g}-\nu-\mathrm{F}_{\mathrm{O}}[\emptyset]$ such that:

$$
\left[\xi_{i} \in \mathrm{op}_{\mathfrak{g}, \nu}\left(\mathcal{O}_{\mathfrak{g}, p}\right)\right] \wedge\left[\xi_{j} \in \mathrm{op}_{\mathfrak{g}, \nu}\left(\mathcal{O}_{\mathfrak{g}, q}\right)\right] .
$$

This shows that $\mathfrak{T}_{\mathfrak{g}}$ is a $\mathfrak{g}-\mathcal{T}_{\mathfrak{g}}^{(\mathrm{H})}$-space $\mathfrak{g}-\mathfrak{T}_{\mathfrak{g}}^{(\mathrm{H})}=\left(\Omega, \mathfrak{g}-\mathcal{T}_{\mathfrak{g}}^{(\mathrm{H})}\right)$.

Let $\left(\xi_{i}, \xi_{j}\right) \in \mathcal{O}_{\mathfrak{g},(p, q)}$ mean $\xi_{i} \in \mathcal{O}_{\mathfrak{g}, p}, \xi_{j} \in \mathcal{O}_{\mathfrak{g}, q}$, and $\left(\xi_{j}, \xi_{i}\right) \notin \mathcal{O}_{\mathfrak{g},(p, q)}$, where $\mathcal{O}_{\mathfrak{g},(p, q)} \in \mathfrak{g}-\nu-\mathrm{F}_{\mathrm{O}}\left[\mathcal{S}_{\mathfrak{g}} \supset \emptyset\right]$. Then, the following relations are easily verified: $\left(\xi_{1}, \xi_{2}\right) \in \mathcal{O}_{\mathfrak{g},(p, q)}$ and $\left(\xi_{2}, \xi_{1}\right) \notin \mathcal{O}_{\mathfrak{g},(p, q)}$ for all $(p, q)=(2,3),(2,7),(6,3),(6,7)$; $\left(\xi_{1}, \xi_{3}\right) \in \mathcal{O}_{\mathfrak{g},(p, q)}$ and $\left(\xi_{3}, \xi_{1}\right) \notin \mathcal{O}_{\mathfrak{g},(p, q)}$ for all $(p, q)=(2,4),(2,7),(5,4),(5,7)$; $\left(\xi_{2}, \xi_{3}\right) \in \mathcal{O}_{\mathfrak{g},(p, q)}$ and $\left(\xi_{3}, \xi_{1}\right) \notin \mathcal{O}_{\mathfrak{g},(p, q)}$ for all $(p, q)=(3,4),(3,6),(5,4),(5,6)$. But, $\mathcal{O}_{\mathfrak{g},(p, q)} \subseteq \operatorname{op}_{\mathfrak{g}, \nu}\left(\mathcal{O}_{\mathfrak{g},(p, q)}\right)$ for all $(p, q)=(2,3),(2,4),(2,7),(3,4),(3,6)$, $(5,4),(5,6),(5,7),(6,3),(6,7)$. Hence, for every $\left(\xi_{i}, \xi_{j}\right) \in \mathfrak{g}-\mathrm{F}_{\mathrm{P}}[\sigma>0]$, there exists $\mathcal{O}_{\mathfrak{g},(p, q)} \in \mathfrak{g}-\nu-\mathrm{F}_{\mathrm{O}}\left[\mathcal{S}_{\mathfrak{g}} \supset \emptyset\right]$ such that:

$$
\left[\left(\xi_{i}, \xi_{j}\right) \in \times_{\lambda=p, q} \mathrm{op}_{\mathfrak{g}, \nu}\left(\mathcal{O}_{\mathfrak{g}, \lambda}\right)\right] \wedge\left[\left(\xi_{i}, \xi_{j}\right) \notin \times_{\lambda=q, p} \mathrm{op}_{\mathfrak{g}, \nu}\left(\mathcal{O}_{\mathfrak{g}, \lambda}\right)\right] .
$$

This shows that $\mathfrak{T}_{\mathfrak{g}}$ is a $\mathfrak{g}-\mathcal{T}_{\mathfrak{g}}^{(\mathrm{F})}$-space $\mathfrak{g}-\mathfrak{T}_{\mathfrak{g}}^{(\mathrm{F})}=\left(\Omega, \mathfrak{g}-\mathcal{T}_{\mathfrak{g}}^{(\mathrm{F})}\right)$.

Let $\left(\xi_{i}, \xi_{j}\right) \in \mathcal{O}_{\mathfrak{g},(p, q)}$ mean $\xi_{i} \in \mathcal{O}_{\mathfrak{g}, p}$ and $\xi_{j} \notin \mathcal{O}_{\mathfrak{g}, p}$, or $\xi_{i} \notin \mathcal{O}_{\mathfrak{g}, p}$ and $\xi_{j} \in \mathcal{O}_{\mathfrak{g}, q}$, where $\mathcal{O}_{\mathfrak{g},(p, q)} \in \mathfrak{g}-\nu-\mathrm{F}_{\mathrm{O}}\left[\mathcal{S}_{\mathfrak{g}} \supset \emptyset\right]$. Then, the following relations are easily verified: $\left(\xi_{1}, \xi_{2}\right) \in \mathcal{O}_{\mathfrak{g},(2,3)}, \mathcal{O}_{\mathfrak{g},(2,7)}, \mathcal{O}_{\mathfrak{g},(6,3)}, \mathcal{O}_{\mathfrak{g},(6,7)} ;\left(\xi_{1}, \xi_{3}\right) \in \mathcal{O}_{\mathfrak{g},(2,4)}, \mathcal{O}_{\mathfrak{g},(2,7)}, \mathcal{O}_{\mathfrak{g},(5,4)}$, $\mathcal{O}_{\mathfrak{g},(5,7)} ;\left(\xi_{2}, \xi_{3}\right) \in \mathcal{O}_{\mathfrak{g},(3,4)}, \mathcal{O}_{\mathfrak{g},(3,6)}, \mathcal{O}_{\mathfrak{g},(5,4)}, \mathcal{O}_{\mathfrak{g},(5,6)}$. But, $\mathcal{O}_{\mathfrak{g},(p, q)} \subseteq \mathrm{op}_{\mathfrak{g}, \nu}\left(\mathcal{O}_{\mathfrak{g},(p, q)}\right)$ for all $(p, q)=(2,3),(2,4),(2,7),(3,4),(3,6),(5,4),(5,6),(5,7),(6,3),(6,7)$. Hence, for every $\left(\xi_{i}, \xi_{j}\right) \in \mathfrak{g}-\mathrm{F}_{\mathrm{P}}[\sigma>0]$, there exists $\mathcal{O}_{\mathfrak{g},(p, q)} \in \mathfrak{g}-\nu-\mathrm{F}_{\mathrm{O}}\left[\mathcal{S}_{\mathfrak{g}} \supset \emptyset\right]$ such that:

$$
\begin{aligned}
{\left[\left(\xi_{i} \in \mathrm{op}_{\mathfrak{g}, \nu}\left(\mathcal{O}_{\mathfrak{g}, p}\right)\right) \wedge\left(\xi_{j} \notin \mathrm{op}_{\mathfrak{g}, \nu}\left(\mathcal{O}_{\mathfrak{g}, p}\right)\right)\right] } & \vee\left[\left(\xi_{i} \notin \mathrm{op}_{\mathfrak{g}, \nu}\left(\mathcal{O}_{\mathfrak{g}, q}\right)\right)\right. \\
& \left.\wedge\left(\xi_{j} \in \mathrm{op}_{\mathfrak{g}, \nu}\left(\mathcal{O}_{\mathfrak{g}, q}\right)\right)\right] .
\end{aligned}
$$

This shows that $\mathfrak{T}_{\mathfrak{g}}$ is a $\mathfrak{g}-\mathcal{T}_{\mathfrak{g}}^{(\mathrm{K})}$-space $\mathfrak{g}-\mathfrak{T}_{\mathfrak{g}}^{(\mathrm{K})}=\left(\Omega, \mathfrak{g}-\mathcal{T}_{\mathfrak{g}}^{(\mathrm{K})}\right)$. 
The elements discussed in the preceding sections can be easily checked from this nice application. In the next section, we provide concluding remarks and future directions of the theory of $\mathfrak{g}-\mathfrak{T}_{\mathfrak{g}}$-separation axioms developed in the earlier sections.

4.3. Concluding Remarks. In this chapter, we developed a new theory, called Theory of $\mathfrak{g}-\mathfrak{T}_{\mathfrak{g}}$-Separation Axioms. The theory is based on the Theory of $\mathfrak{g}$ - $\mathfrak{T}_{\mathfrak{g}}$-Sets but not on the Theory of $\mathfrak{g}-\mathfrak{T}_{\mathfrak{g}}$-Maps. In its own rights, the proposed theory has several advantages. The very first advantage is that the theory holds equally well when $\left(\Omega, \mathcal{T}_{\mathfrak{g}}\right)=(\Omega, \mathcal{T})$ and other characteristics adapted on this ground, in which case it might be called Theory of $\mathfrak{g}-\mathfrak{T}$-Separation Axioms.

Thus, in a $\mathcal{T}_{\mathfrak{g}}$-space the proposed theoretical framework categorises each element of the quintuple sequence $\left\langle\mathfrak{g}-\mathrm{T}_{\mathfrak{g}, \alpha}\right\rangle_{\alpha \in \Lambda}$ as $\mathfrak{g}$-T $\mathfrak{g}, \alpha$-axioms of type $\alpha$ and of categories $\nu$, where $(\alpha, \nu) \in \Lambda \times I_{3}^{0}$ and $\Lambda=\{\mathrm{K}, \mathrm{F}, \mathrm{H}, \mathrm{R}, \mathrm{N}\}$ and theorises the concepts in a unified way; in a $\mathcal{T}$-space it categorises each element of the quintuple sequence $\left\langle\mathfrak{g}-\mathrm{T}_{\alpha}\right\rangle_{\alpha \in \Lambda}$ as $\mathfrak{g}$ - $\mathrm{T}_{\alpha}$-axioms of type $\alpha$ and of categories $\nu$, where $(\alpha, \nu) \in \Lambda \times I_{3}^{0}$ and $\Lambda=\{\mathrm{K}, \mathrm{F}, \mathrm{H}, \mathrm{R}, \mathrm{N}\}$ and theorises the concepts in a unified way.

Since the theory of $\mathfrak{g}-\mathfrak{T}_{\mathfrak{g}}$-separation Axioms has been based solely on theory of $\mathfrak{g}-\mathfrak{T}_{\mathfrak{g}}$-sets, as pointed out above, it is an interesting topic for future research either to develop the theory of $\mathfrak{g}-\mathfrak{T}_{\mathfrak{g}}$-separation axioms of mixed categories based on the aforementioned theory or to develop it but based on the theory of $\mathfrak{g}$ - $\mathfrak{T}_{\mathfrak{g}}$-maps. More precisely, either for some pair $(\nu, \mu) \in I_{3}^{0} \times I_{3}^{0}$ such that $\nu \neq \mu$, to develop the theory of $\mathfrak{g}$ - $\mathfrak{T}_{\mathfrak{g}}$-separation axioms based on the theory of $\mathfrak{g}$ - $\mathfrak{T}_{\mathfrak{g}}$-open sets belonging to the class $\left\{\mathcal{O}_{\mathfrak{g}}=\mathcal{O}_{\mathfrak{g}, \nu} \cup \mathcal{O}_{\mathfrak{g}, \mu}:\left(\mathcal{O}_{\mathfrak{g}, \nu}, \mathcal{O}_{\mathfrak{g}, \mu}\right) \in \mathfrak{g}-\nu-\mathrm{O}\left[\mathfrak{T}_{\mathfrak{g}}\right] \times \mathfrak{g}-\mu-\mathrm{O}\left[\mathfrak{T}_{\mathfrak{g}}\right]\right\}$ and the theory of $\mathfrak{g}-\mathfrak{T}_{\mathfrak{g}}-$ closed sets belonging to the class $\left\{\mathcal{K}_{\mathfrak{g}}=\mathcal{K}_{\mathfrak{g}, \nu} \cup \mathcal{K}_{\mathfrak{g}, \mu}:\left(\mathcal{K}_{\mathfrak{g}, \nu}, \mathcal{K}_{\mathfrak{g}, \mu}\right) \in \mathfrak{g}-\nu-\mathrm{K}\left[\mathfrak{T}_{\mathfrak{g}}\right] \times\right.$ $\left.\mathfrak{g}-\mu-\mathrm{K}\left[\mathfrak{T}_{\mathfrak{g}}\right]\right\}$ in a $\mathcal{T}_{\mathfrak{g}}$-space $\mathfrak{T}_{\mathfrak{g}}$ or, to develop the theory of $\mathfrak{g}$ - $\mathfrak{T}_{\mathfrak{g}}$-separation axioms based on the theory of $\mathfrak{g}-\mathfrak{T}_{\mathfrak{g}}$-maps, called $\mathfrak{g}$ - $\left(\mathfrak{T}_{\Lambda}, \mathfrak{T}_{\Theta}\right)$-continuous maps, $\mathfrak{g}$ - $\left(\mathfrak{T}_{\Lambda}, \mathfrak{T}_{\Theta}\right)$ irresolute maps and $\mathfrak{g}$ - $\left(\mathfrak{T}_{\Lambda}, \mathfrak{T}_{\Theta}\right)$-homeomorphism maps, where $\Lambda, \Theta \in\{\Omega, \Sigma, \Upsilon\}$,

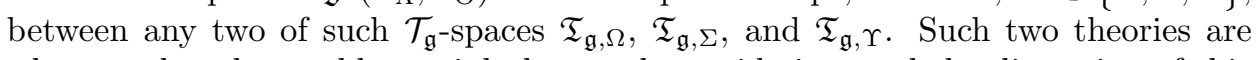
what we thought would certainly be worth considering, and the discussion of this chapter ends here.

\section{REFERENCES}

1. F. G. Arenas, J. Dontchev, and M. L. Puertas, Unification Approach to the Separation Axioms Between $\mathrm{T}_{0}$ and Completely Hausdorff, acta math. hungar., vol. 86 (1-2), pp. 75-82, 2000.

2. S. Bayhan, A. Kanibir, and I. L. Reilly, On Functions between Generalized Topological Spaces, appl. gen. topol., vol. 14 (2), pp. 195-203, 2013.

3. C. Boonpok, On Generalized Continuous Maps in Čech Closure Spaces, general mathematics, vol. 19 (3), pp. 3-10, 2011.

4. C. Carlos, R. Namegalesh, and R. Ennis, Separation Axiom on Enlargements of Generalized Topologies, revista integración, vol. 32 (1), pp. 19-26, 2014.

5. Á. Császár, Separations Axioms for Generalized Topologies, acta math. hungar., vol. 104 (1-2), pp. 63-69, 1998.

6. _— Generalized Open Sets in Generalized Topologies, acta math. hungar., vol. 106 (1-2), pp. 53-66, 2005.

7. __ Remarks on Quasi-Topologies, acta math. hungar., vol. 119 (1-2), pp. 197-200, 2008.

8. A. Danabalan and C. Santhi, A Class of Separation Axioms in Generalized Topology, mathematical journal of interdisciplinary sciences, vol. 4 (2), pp. 151-159, 2016.

9. M. M. Deza and E. Deza, Encyclopedia of Distances, discrete mathematics, Springer-Verlag Berlin , 1st Ed., 2009.

10. J. Dontchev, On Some Separation Axioms Associated with the $\alpha$-Topology, mem. fac. sci. kochi univ. ser. a, math., vol. 18, pp. 31-35, 1997. 
11. K. El-Saady and F. Al-Nabbat, Separation Axioms in Generalized Base Spaces, british journal of mathematics and computer science, vol. 17 (2), pp. 1-8, 2016.

12. R. Ennis, C. Carlos, and S. José, $\gamma-(\alpha, \beta)$-Semi Sets and New Generalized Separation Axioms, bull. malays. sci. soc. (2), vol. 30 (1), pp. 13-21, 2007.

13. A. Keskin and T. Noiri, Higher Separation Axioms via Semi*-Open Sets, bulletin of the iranian mathematical society, vol. 35 (1), pp. 179-198, 2009.

14. L. L. L. Lusanta and H. M. Rara, Generalized Star $\alpha-$ b-Separation Axioms in Bigeneralized Topological Spaces, applied mathematical sciences, vol. 9 (75), pp. 3725-3737, 2015.

15. A. S. Mashhour, I. A. Hasanein, and S. N. E. Deeb, $\alpha$-Continuous and $\alpha$-Open Mappings, acta. math. hungar., vol. 41 (3-4), pp. 213-218, 1983.

16. M. V. Mielke, Separation Axioms and Geometric Realizations, indian j. pure appl. math., vol. 25 (7), pp. 711-722, 1994.

17. W. K. Min, Remarks on Separation Axioms on Generalized Topological Spaces, journal of the chungcheong mathematical society, vol. 23 (2), pp. 293-298, 2010.

18. S. P. Missier and A. Robert, Higher Separation Axioms via Semi*-Open Sets, int. journal of engineering and science, vol. 4 (6), pp. 37-45, 2014.

19. D. Narasimhan, An Overview of Separation Axioms in Recent Research, international journal of pure and applied mathematics, vol. 76 (4), pp. 529-548, 2012.

20. V. Pankajam and D. Sivaraj, Some Separation Axioms in Generalized Topological Spaces, bol. soc. paran. mat., vol. 31 (1), pp. 29-42, 2013.

21. V. Pavlović and A. S. Cvetković, On Generalized Topologies arising from Mappings, vesnik, vol. 38 (3), pp. 553-565, 2012.

22. D. Pratulananda and A. R. Mamun, $g^{*}$-Closed Sets and a New Separation Axiom in Alexandroff Spaces, archivum mathematicum (brno), vol. 39, pp. 299-307, 2003.

23. T. C. K. Raman, V. Kumari, and M. K. Sharma, $\alpha$-Generalized and $\alpha^{*}$-Separation Axioms for Topological Spaces, iosr journal of mathematics, vol. 10 (3), pp. 32-36, 2014.

24. A. D. Ray and R. Bhowmick, Separation Axioms on Bi-Generalized Topological Spaces, journal of the chungcheong mathematical society, vol. 27 (3), pp. 363-379, 2014.

25. B. Roy and R. Sen, Applications of Maximal $\mu$-Open Sets in Generalized Topology and Quasi Topology, general algebra and applications, vol. 33, pp. 129-135, 2013.

26. M. S. Sarsak, New Separation Axioms in Generalized Topological Spaces, acta math. hungar., vol. 132 (3), pp. 244-252, 2011.

27. L. A. Steen and J. A. Jr. Seebach, Counterexamples in Topology, separation axioms, Springer, New York, pp. 11-17, 1978.

28. J. Thomas and S. J. John, Soft Generalized Separation Axioms in Soft Generalized Topological Spaces, international journal of scientific and engineering research, vol. 6 (3), pp. 969-974, 2015.

Dr. Mohammad Irshad KHODABOCUS

Current address: Department of Mathematics, Faculty of Science, University of Mauritius

E-mail address: ikhodabo@gmail.com

Dr. NoOR-Ul-Hacq SOOKIA

Current address: Department of Mathematics, Faculty of Science, University of Mauritius

E-mail address: sookian@uom.ac.mu 